\title{
HEAT KERNEL AND GREEN FUNCTION ESTIMATES ON NONCOMPACT SYMMETRIC SPACES
}

\author{
J.-P. ANKER AND L. JI \\ In memory of Carl S. Herz (1930-1995)
}

\section{Introduction}

Let $\mathbb{X}=G / K$ be a noncompact Riemannian symmetric space. Although basic harmonic analysis on $\mathbb{X}$ has been settled in the sixties (see [GV], $[\mathrm{He} 2,3]$, for thorough presentations of this material), it is only recently that it has been used efficiently to produce sharp and complete results comparable to the Euclidean or the compact case ([An3,5], [BrOSc], [CowGM1,2], $[\mathrm{MaNSt}],[\mathrm{Str}], \ldots)$. The reason may be that time was needed to digest and refine the formidable work of Harish-Chandra and his followers.

Our main object of study in this paper is the heat kernel $h_{t}(x, y)=$ $h_{t}\left(y^{-1} x\right)$ on $\mathbb{X}$, for which we produce optimal upper and lower bounds, as well as asymptotics at infinity, in what is arguably the most interesting case, namely when the time variable $t$ is larger than (any constant times) the distance $d(x, y)$ between the space variables. The upper bound was conjectured some years ago by the first author [An2] and proves amazingly to be a lower bound too. The restriction $1+t \geq$ const. $d(x, y)$ in our results is due to a lack of control in the Trombi \& Varadarajan expansion for spherical functions along the walls. Fortunately this is no problem in all applications since good upper bounds, with fast decaying Gaussian factors $e^{- \text {const. } d(x, y)^{2} / t}$, are known to hold in the remaining domain $t \ll d(x, y)$.

Such heat kernel estimates have several important consequences. Among them let us mention the exact behavior of the Green function, which is fully obtained for the first time on general symmetric spaces $\mathbb{X}$. Recall that this is precisely the analytic information needed for a complete description of

First author partially supported by the European Commission (HCM 1994-1997 Network Fourier Analysis and TMR 1998-2001 Network Harmonic Analysis). Second author partially supported by the U.S.A. National Science Foundation (postdoctoral fellowship DMS 9407427 and grant DMS 9704434.) 
the Martin boundary of $\mathbb{X}([\mathrm{Gu}],[\mathrm{GuJT} 1,2])$. Other consequences worth mentioning are on one hand optimal kernel bounds for the Poisson semigroup $e^{-t \sqrt{-\Delta}}$ on $\mathbb{X}$ and on the other hand a delicate maximal inequality for a particular heat diffusion on the Iwasawa component $S=(\exp \mathfrak{a}) N$ of $G$, which was sought after in [CowGGM].

Our paper is organized as follows. Section 2 contains some necessary reminders about symmetric spaces and spherical analysis, notably spherical function asymptotics. In section 3 we establish our main result, namely the above mentioned bounds for the heat kernel on $\mathbb{X}$. Section 4 is devoted to some applications: $L^{p}$ heat propagation on $\mathbb{X}$, optimal bounds for the Bessel-Green-Riesz kernels (in particular for the Green function) and for the Poisson kernel on $\mathbb{X}$, and finally the weak $L^{1} \rightarrow L^{1}$ boundedness alluded to above of the heat maximal operator associated to a distinguished Laplacian on $S$. In section 5 we refine our previous results by obtaining asymptotics at infinity for the various kernels considered before. Historical comments will be made throughout the text.

The main results in this paper were announced in [AnJ1] and in several talks during the past years. Both authors would like to thank M. Babillot, Y. Guivarc'h and J. C. Taylor for helpful discussions and indications. The first author enjoyed hospitality at the University of Wrocław, at the University of Wisconsin in Madison, and at the Mittag-Leffler Institute, where substantial parts of this article were carried out.

\section{Preliminaries}

In this section we recall the basic material about noncompact Riemannian symmetric spaces and spherical analysis thereupon which will be used throughout the article. We shall seize the opportunity of setting up the notation. The book [GV] will serve as our main reference.

2.1 Noncompact Riemannian symmetric spaces. $G$ will denote a noncompact reductive Lie group in Harish-Chandra's class, $K$ a maximal compact subgroup, $\theta$ a corresponding Cartan involution, $\mathfrak{g}=\mathfrak{k} \oplus \mathfrak{p}$ the resulting decomposition on the Lie algebra level, and $\mathbb{X}=G / K$ the associated Riemannian symmetric space with nonpositive curvature. It is understood that $\mathfrak{g}$ is equipped with an admissible nondegenerate bilinear form $B$ (coinciding with the Killing form on $\mathfrak{g}^{\prime}=[\mathfrak{g}, \mathfrak{g}]$ ) and $\mathbb{X}$ with the associated $G$-invariant metric. Using the inner product

$$
\langle X, Y\rangle=-B(X, \theta(Y))
$$


we shall systematically identify any subspace of $\mathfrak{g}$ with its dual space. (Recall that the Harish-Chandra class is the natural setting for this theory. Actually, as long as the associated symmetric spaces are concerned, the difference with the semisimple setting consists only in possible additional Euclidean factors. Specifically let $G^{\prime}$ be the analytic subgroup of $G$ corresponding to $\mathfrak{g}^{\prime}=[\mathfrak{g}, \mathfrak{g}], K^{\prime}=K \cap G^{\prime}$, and $V=\exp (\mathfrak{a} \cap \mathfrak{z})$, where $\mathfrak{z}$ denotes the center of $\mathfrak{g}$. Then $G^{\prime}$ is closed, semisimple, with finite center, $K^{\prime}$ is a maximal compact subgroup of $G^{\prime}, V$ is a split component of $G$, and $\mathbb{X}$ splits as the product of the Riemannian symmetric space of noncompact type $\mathbb{X}^{\prime}=G^{\prime} / K^{\prime}$ times the Euclidean space $V \cong \mathfrak{a} \cap \mathfrak{z}$. Similarly $M^{F} / K_{F} \cong G_{F} /\left(G_{F} \cap K\right) \times \mathfrak{a}^{F}$, where $G_{F}$ denotes the analytic subgroup of $G$ corresponding to $\left[\mathfrak{m}^{F}, \mathfrak{m}^{F}\right]=\left[\mathfrak{m}_{F}, \mathfrak{m}_{F}\right]$.)

Given a Cartan subspace $\mathfrak{a}$ in $\mathfrak{p}, A=\exp \mathfrak{a}$ denotes the corresponding analytic subgroup of $G, M$ its centralizer in $K, M^{\prime}$ its normalizer in $K$, $\Sigma$ the restricted root system of $(\mathfrak{g}, \mathfrak{a})$, and $W$ the associated Weyl group. Once a positive Weyl chamber $\mathfrak{a}^{+}$has been selected, $\Sigma^{+}$(resp. $\Sigma^{++}$or $\Sigma^{+++}$) denotes the corresponding set of positive (resp. positive indivisible or simple) roots, $\mathfrak{n}$ the direct sum of all positive root subspaces $\mathfrak{g}_{\alpha}, N=$ $\exp \mathfrak{n}$ the corresponding analytic subgroup of $G$, and $\varrho$ the half sum of all positive roots $\alpha$ counted with their multiplicities $m_{\alpha}=\operatorname{dim} \mathfrak{g}_{\alpha}$. Let $n$ be the dimension of $\mathbb{X}, \ell$ its rank (i.e. the dimension of $\mathfrak{a}$ ), and $m=\sum_{\alpha \in \Sigma^{+}} m_{\alpha}$ the dimension of $N$, so that $n=\ell+m$. Recall the decompositions

$$
\begin{cases}G=K(\exp \mathfrak{a}) N & (\text { Iwasawa }), \\ G=K\left(\exp \overline{\mathfrak{a}^{+}}\right) K & (\text { Cartan }) .\end{cases}
$$

Denote by $H(x) \in \mathfrak{a}$ and $x^{+} \in \overline{\mathfrak{a}^{+}}$the middle components of $x \in G$ in these decompositions, and by $|x|=\left|x^{+}\right|$the distance to the origin.

Lemma 2.1.2. $d(x K, y K) \geq\left|x^{+}-y^{+}\right| \forall x, y \in G$.

Let us give a proof of this elementary result, for lack of known reference (see [Cl, Lemma 6.3] for the analog in the compact Lie group setting). Since

$$
d((\exp X) K,(\exp Y) K) \geq|X-Y|, \quad \forall X, Y \in \mathfrak{p}
$$

(see for instance [He1, Theorem I.13.1]), we can reduce to the corresponding inequality in the flat case, namely

$$
|X-Y| \geq\left|X^{+}-Y^{+}\right|, \quad \forall X, Y \in \mathfrak{p}
$$

with obvious notation. Writing $X=\operatorname{Ad} k_{1} \cdot X^{+}, Y=\operatorname{Ad} k_{2} \cdot Y^{+}$with $k_{1}, k_{2} \in K$, we can furthermore restrict to $X^{+}, Y^{+} \in \mathfrak{a}^{+}$and $k_{1}$ or $k_{2}=e$. Let us show that the minimum of $f(k)=\left|\operatorname{Ad} k \cdot X^{+}-Y^{+}\right|$is necessarily reached on $M^{\prime}$. If $k_{0}$ is a local extremum of $f$, we have indeed 


$$
\begin{aligned}
0=\left.\frac{d}{d t}\right|_{0} f\left(k_{0} \exp t Z\right)^{2} & =2\left\langle\operatorname{Ad} k_{0} \cdot X^{+}-Y^{+}, \operatorname{Ad} k_{0} \cdot\left[Z, X^{+}\right]\right\rangle \\
& =2\left\langle\left[X^{+}, \operatorname{Ad} k_{0}^{-1} \cdot Y^{+}\right], Z\right\rangle
\end{aligned}
$$

for every $Z \in \mathfrak{k}$, which implies successively $\left[X^{+}, \operatorname{Ad} k_{0}^{-1} \cdot Y^{+}\right]=0$, $\operatorname{Ad} k_{0}^{-1} . Y^{+} \in \mathfrak{a}$ and $k_{0} \in M^{\prime}$. Finally it is well known that $\min _{w \in W}\left|w \cdot X^{+}-Y^{+}\right|$ $=\left|X^{+}-Y^{+}\right|$, and this concludes the proof of Lemma 2.1.2.

Let us fix the invariant measures on the groups and homogeneous spaces introduced so far. $K$ is equipped with its normalized Haar measure, a, $N$, $G / K$ and $K / M$ with the invariant measures induced by the inner product (2.1.1), and $G$ with

$$
\int_{G} d x f(x)=\int_{G / K} d(x K) \int_{K} d k f(x k) .
$$

Thus the Haar measure on $G$ writes

$$
\int_{G} d x f(x)=2^{-\frac{m}{2}} \int_{K} d k \int_{\mathfrak{a}} d H e^{2\langle\varrho, H\rangle} \int_{N} d n f(k(\exp H) n)
$$

in the Iwasawa decomposition and

$$
\begin{array}{r}
\int_{G} d x f(x)=|K / M| \int_{K} d k_{1} \int_{\mathfrak{a}^{+}} d H \boldsymbol{\delta}(H) \int_{K} d k_{2} f\left(k_{1}(\exp H) k_{2}\right), \\
\boldsymbol{\delta}(H)=\prod_{\alpha \in \Sigma^{+}} \sinh ^{m_{\alpha}}\langle\alpha, H\rangle \asymp\left\{\prod_{\alpha \in \Sigma^{+}}\left(\frac{\langle\alpha, H\rangle}{1+\langle\alpha, H\rangle}\right)^{m_{\alpha}}\right\} e^{2\langle\varrho, H\rangle,}
\end{array}
$$

in the Cartan decomposition. (The symbol $\asymp$ means precisely that there exist two constants $0<C_{1} \leq C_{2}<+\infty$ such that $C_{1}\left\{\prod_{\alpha \in \Sigma^{+}}\left(\frac{\langle\alpha, H\rangle}{1+\langle\alpha, H\rangle}\right)^{m_{\alpha}}\right\} e^{2\langle\varrho, H\rangle}$ $\leq \boldsymbol{\delta}(H) \leq C_{2}\left\{\prod_{\alpha \in \Sigma^{+}}\left(\frac{\langle\alpha, H\rangle}{1+\langle\alpha, H\rangle}\right)^{m_{\alpha}}\right\} e^{2\langle\varrho, H\rangle}, \forall H \in \mathfrak{a}^{+}$.) The volume of $K / M$ can be computed explicitly (see (2.2.3) and (2.2.4) below). Notice the differences with Harish-Chandra's conventions, where the Lebesgue measure on $\mathfrak{a}$ is divided by $(2 \pi)^{\ell / 2}$, the Haar measure on $N$ is normalized by the condition $\int_{N} d n e^{-2\langle\varrho,(H \circ \theta)(n)\rangle}=1$, and the Haar measure on $G$ is given by $d x=d k e^{2\langle\varrho, H\rangle} d H d n$ in the Iwasawa decomposition.

Let us next describe the various faces of $\overline{\mathfrak{a}^{+}}$and the associated standard parabolic subgroups of $G$. They are in 1-1 correspondence with the subsets $F$ in $\Sigma^{+++}$. Specifically denote by $\Sigma_{F}^{(+)}$the (positive) root subsystem generated by $F$. Furthermore split

$$
\mathfrak{a}=\mathfrak{a}_{F} \oplus \mathfrak{a}^{F}, \quad \mathfrak{n}=\mathfrak{n}_{F} \oplus \mathfrak{n}^{F} \quad \text { and } \quad N=N_{F} N^{F} \quad \text { accordingly, }
$$

where $\mathfrak{a}_{F}$ is the subspace generated by $F, \mathfrak{a}^{F}$ its orthogonal in $\mathfrak{a}, \mathfrak{n}_{F}=$ $\bigoplus_{\alpha \in \Sigma_{F}^{+}} \mathfrak{g}_{\alpha}$, and $\mathfrak{n}^{F}=\bigoplus_{\alpha \in \Sigma^{+} \backslash \Sigma_{F}^{+}} \mathfrak{g}_{\alpha}$. Then the face of $\overline{\mathfrak{a}^{+}}$and the standard parabolic subgroup attached to $F$ are respectively

$$
\left(\mathfrak{a}^{F}\right)^{+}=\left\{H \in \mathfrak{a}^{F} \mid\langle\alpha, H\rangle>0, \forall \alpha \in \Sigma^{+++} \backslash F\right\}
$$


and the normalizer $P_{F}$ of $N^{F}$ in $G$.

We shall write $H=H_{F}+H^{F}$ according to the decomposition $\mathfrak{a}=$ $\mathfrak{a}_{F} \oplus \mathfrak{a}^{F}$. For example

$$
\varrho=\varrho_{F}+\varrho^{F}, \quad \text { with } \varrho_{F}=\frac{1}{2} \sum_{\alpha \in \Sigma_{F}^{+}} m_{\alpha} \alpha \quad \text { and } \quad \varrho^{F}=\frac{1}{2} \sum_{\alpha \in \Sigma^{+} \backslash \Sigma_{F}^{+}} m_{\alpha} \alpha .
$$

Similarly

$$
\ell=\ell_{F}+\ell^{F}, \quad \text { with } \quad \ell_{F}=\operatorname{dim} \mathfrak{a}_{F} \quad \text { and } \quad \ell^{F}=\operatorname{dim} \mathfrak{a}^{F} .
$$

$P_{F}$ has Langlands decomposition $P_{F}=M_{F}\left(\exp \mathfrak{a}^{F}\right) N^{F} . M_{F}$ and $M^{F}=$ $M_{F}\left(\exp \mathfrak{a}^{F}\right)$ are closed subgroups of $G$, which belong to the Harish-Chandra class and are $\theta$-stable. $K_{F}=K \cap M_{F}=K \cap M^{F}$ is a joint maximal compact subgroup. With the obvious notation, $\mathfrak{a}_{F}$ and $\mathfrak{a}$ are Cartan subspaces for $\mathfrak{m}_{F}$ and $\mathfrak{m}^{F}=\mathfrak{m}_{F} \oplus \mathfrak{a}^{F}$ respectively, $\Sigma_{F}$ is the corresponding root system, and its Weyl group $W_{F}$ is the stabilizer in $W$ of any element $H \in\left(\mathfrak{a}^{F}\right)^{+}$.

We conclude this subsection with some less standard results about the geometry of $\mathfrak{a}$.

Lemma 2.1.6. (i) $|\langle\alpha, H\rangle| \leq|H| \forall \alpha \in \Sigma, \forall H \in \mathfrak{a}$.

(ii) There exists a positive constant $c_{1}$ such that

$$
|H| \leq c_{1} \max _{\alpha \in F}|\langle\alpha, H\rangle| \quad \forall H \in \mathfrak{a}_{F} \text { and } \forall F \subset \Sigma^{+++} .
$$

Proof. (i) Decompose $H=H_{\Sigma^{+++}}+H^{\Sigma^{+++}}$. Then

$$
\begin{aligned}
|H|^{2} \geq\left|H_{\Sigma^{++}}\right|^{2}=\operatorname{tr}\left(\operatorname{ad}_{\mathfrak{g}}^{2} H_{\Sigma^{+++}}\right) & =2 \sum_{\alpha \in \Sigma^{+}} m_{\alpha}\left\langle\alpha, H_{\Sigma^{++}}\right\rangle^{2} \\
& =2 \sum_{\alpha \in \Sigma^{+}} m_{\alpha}\langle\alpha, H\rangle^{2} \geq 2\langle\alpha, H\rangle^{2} .
\end{aligned}
$$

(ii) Since $F$ is a basis of $\mathfrak{a}_{F}, H \mapsto \max _{\alpha \in F}|\langle\alpha, H\rangle|$ is a norm on $\mathfrak{a}_{F}$. Hence there exists a positive constant $C_{F}$ such that

$$
\max _{\alpha \in F}|\langle\alpha, H\rangle| \leq C_{F}|H| \quad \forall H \in \mathfrak{a}_{F} .
$$

We obtain (ii) by taking for $c_{1}$ the maximum of all constants $C_{F}$.

Assume temporarily that $\mathbb{X}$ has no split component (i.e. $\Sigma$ generates $\mathfrak{a}$ ) and consider the cones

$$
\begin{aligned}
\overline{\mathfrak{a}^{+}}(F, \delta, \varepsilon)=\left\{H \in \overline{\mathfrak{a}^{+}}|\langle\alpha, H\rangle \leq \delta| H \mid \forall \alpha \in F\right. \\
\left.\quad \text { and }\langle\alpha, H\rangle \geq \varepsilon|H| \forall \alpha \in \Sigma^{+++} \backslash F\right\},
\end{aligned}
$$

where $F$ is a subset of $\Sigma^{+++}$and $0<\delta \leq \varepsilon<+\infty$.

Lemma 2.1.7. (i) $\left|H_{F}\right| \leq c_{1} \delta|H|$ if $H \in \overline{\mathfrak{a}^{+}}(F, \delta, \varepsilon)$.

(ii) $\overline{\mathfrak{a}^{+}}$can be covered with $2^{\ell}-1$ subcones $\overline{\mathfrak{a}^{+}}\left(F, \delta_{F}, \varepsilon_{F}\right)$, where $F$ ranges through the proper subsets of $\Sigma^{+++}$and $\delta_{F}, \delta_{F} / \varepsilon_{F}$ are arbitrarily small. 
Proof. The statement (i) follows from Lemma 2.1.6.ii and the definition of $\overline{\mathfrak{a}^{+}}(F, \delta, \varepsilon)$ :

$$
\left|H_{F}\right| \leq c_{1} \max _{\alpha \in F}\left|\left\langle\alpha, H_{F}\right\rangle\right|=c_{1} \max _{\alpha \in F}|\langle\alpha, H\rangle| \leq c_{1} \delta|H| .
$$

(ii) Observe first that one can restrict by homogeneity to the unitsphere $\mathrm{S}(\mathfrak{a})=\{H \in \mathfrak{a}|| H \mid=1\}$. For $\varepsilon>0$ small enough, the $\ell$ hyperplanes $\langle\alpha, H\rangle=\varepsilon\left(\alpha \in \Sigma^{+++}\right)$divide the (spherical) domain $\mathrm{S}\left(\overline{\mathfrak{a}^{+}}\right)=$ $\left\{H \in \overline{\mathfrak{a}^{+}}|| H \mid=1\right\}$ into $2^{\ell}-1$ (spherical) subdomains, defined by the conditions

$$
\begin{cases}\langle\alpha, H\rangle \leq \varepsilon & \forall \alpha \in F \\ \langle\alpha, H\rangle \geq \varepsilon & \forall \alpha \in \Sigma^{+++} \backslash F\end{cases}
$$

and indexed by the proper subsets $F$ of $\Sigma^{+++}$. Given such an $\varepsilon$ and $0<\gamma<1$, set $\delta_{F}=\gamma^{\ell-|F|} \varepsilon$ and $\varepsilon_{F}=\gamma^{\ell-|F|-1} \varepsilon$. Then $\delta_{F} / \varepsilon_{F}=\gamma$ and one shows by (backward) induction on $k=\ell-1, \ell-2, \ldots, 1,0$ that $\bigcup_{k \leq|F|<\ell} \mathrm{S}\left(\overline{\mathfrak{a}^{+}}\right)\left(F, \delta_{F}, \varepsilon_{F}\right)$ (obvious notation) contains

$$
\left\{H \in \mathrm{S}\left(\overline{\mathfrak{a}^{+}}\right) \mid\langle\alpha, H\rangle \leq \gamma^{\ell-k} \varepsilon \text { for at least } k \text { simple roots } \alpha\right\} .
$$

For $k=0$ this amounts to $\bigcup_{F \varsubsetneqq \Sigma^{+++}} \overline{\mathfrak{a}^{+}}\left(F, \delta_{F}, \varepsilon_{F}\right)=\overline{\mathfrak{a}^{+}}$.

2.2 Spherical analysis. The role played by exponentials in Euclidean Fourier analysis is played by the (elementary) spherical functions in the Fourier analysis of bi- $K$-invariant functions on $G$. Recall Harish-Chandra's integral formula

$$
\varphi_{\lambda}(x)=\int_{K} d k e^{\langle i \lambda-\varrho, H(x k)\rangle}
$$

for these functions and the definition of the spherical Fourier transform

$$
\mathcal{H} f(\lambda)=\int_{G} d x f(x) \varphi_{-\lambda}(x) .
$$

Among its mapping properties we shall use the following result.

Theorem 2.2.2 ([GV, Theorem 6.4.1], see also [An4]). (i) $\mathcal{H}$ is a (topological) isomorphism between the Schwartz spaces $\mathcal{S}(G)^{\natural}$ and $\mathcal{S}(\mathfrak{a})^{W}$.

(ii) Moreover we have the inversion formula

$$
f(x)=\frac{c_{2}}{|W|} \int_{\mathfrak{a}} \frac{d \lambda}{|\mathbf{c}(\lambda)|^{2}} \mathcal{H} f(\lambda) \varphi_{\lambda}(x)
$$

with $c_{2}=2^{m} /(2 \pi)^{\ell}|K / M|$.

This statement requires some explanations. The $\left(L^{2}\right)$ Schwartz space $\mathcal{S}(G)$ consists of all functions $f \in \mathcal{C}^{\infty}(G)$ satisfying

$$
\sup _{k_{1} \in K, H \in \overline{\mathfrak{a}}^{+}, k_{2} \in K}(1+|H|)^{N} e^{\langle\varrho, H\rangle}\left|f\left(D_{1}: k_{1}(\exp H) k_{2}: D_{2}\right)\right|<+\infty
$$


for every $D_{1}, D_{2} \in \mathrm{U}(\mathfrak{g})$ and $N \geq 0$, where $f\left(D_{1}: x: D_{2}\right)$ denotes left and right differentiation of $f$ at $x \in G$ with respect to the elements $D_{1}$ and $D_{2}$ in the universal enveloping algebra $\mathrm{U}(\mathfrak{g})$. The superscript $\downarrow$ denotes the subspace of bi- $K$-invariant functions. Similarly $\mathcal{S}(\mathfrak{a})^{W}$ is the subspace of $W$-invariants in the classical Schwartz space on $\mathfrak{a}$.

$\mathbf{c}(\lambda)=\frac{\mathbf{I}(\lambda)}{\mathbf{I}(-i \varrho)}, \quad$ where $\quad \mathbf{I}(\lambda)=\int_{N} d n e^{-\langle i \lambda+\varrho,(H \circ \theta)(n)\rangle} \quad$ when $\lambda \in \mathfrak{a}-i \mathfrak{a}^{+}$, is the famous c-function of Harish-Chandra, which was determined explicitly by Gindikin \& Karpelevič. By resuming their computation carefully as in $[\mathrm{DuKoV}, \mathrm{pp} .43-50]$ or in $[\mathrm{Mn}]$, one obtains for our choice of invariant measures

$$
\mathbf{I}(\lambda)=\prod_{\alpha \in \Sigma^{++}} I_{\alpha}\left(\frac{\langle\alpha, \lambda\rangle}{\langle\alpha, \alpha\rangle}\right)
$$

with

$$
I_{\alpha}(\nu)=\left(\frac{\sqrt{2 \pi}}{|\alpha|}\right)^{m_{\alpha}} \frac{\Gamma(i \nu)}{\Gamma\left(i \nu+\frac{1}{2} m_{\alpha}\right)} \times\left(\frac{\sqrt{2 \pi}}{2|\alpha|}\right)^{m_{2 \alpha}} \frac{\Gamma\left(\frac{i}{2} \nu+\frac{1}{4} m_{\alpha}\right)}{\Gamma\left(\frac{i}{2} \nu+\frac{1}{4} m_{\alpha}+\frac{1}{2} m_{2 \alpha}\right)} .
$$

As a consequence

$$
\begin{gathered}
\mathbf{c}(\lambda)=\prod_{\alpha \in \Sigma^{+}} c_{\alpha}\left(\frac{\langle\alpha, \lambda\rangle}{\langle\alpha, \alpha\rangle}\right), \quad \text { where } \\
c_{\alpha}(\nu)=\frac{\Gamma\left(\frac{\langle\alpha, \varrho\rangle}{\langle\alpha, \alpha\rangle}+\frac{1}{2} m_{\alpha}\right)}{\Gamma\left(\frac{\langle\alpha, \varrho\rangle}{\langle\alpha, \alpha\rangle}\right)} \frac{\Gamma\left(\frac{1}{2} \frac{\langle\alpha, e\rangle}{\langle\alpha, \alpha\rangle}+\frac{1}{4} m_{\alpha}+\frac{1}{2} m_{2 \alpha}\right)}{\Gamma\left(\frac{1}{2} \frac{\langle\alpha, e\rangle}{\langle\alpha, \alpha\rangle}+\frac{1}{4} m_{\alpha}\right)} \frac{\Gamma(i \nu)}{\Gamma\left(i \nu+\frac{1}{2} m_{\alpha}\right)} \frac{\Gamma\left(\frac{i}{2} \nu+\frac{1}{4} m_{\alpha}\right)}{\Gamma\left(\frac{i}{2} \nu+\frac{1}{4} m_{\alpha}+\frac{1}{2} m_{2 \alpha}\right)} .
\end{gathered}
$$

Formula (2.2.3) is also useful to evaluate

$$
|K / M|=2^{m / 2} \mathbf{I}(-i \varrho)
$$

We shall often consider the expression

where $\boldsymbol{\pi}(i \lambda)=\prod_{\alpha \in \Sigma^{++}}\langle\alpha, \lambda\rangle$ and

$$
\mathbf{b}(\lambda)=\boldsymbol{\pi}(i \lambda) \mathbf{c}(\lambda)=\prod_{\alpha \in \Sigma^{++}} b_{\alpha}\left(\frac{\langle\alpha, \lambda\rangle}{\langle\alpha, \alpha\rangle}\right)
$$

$$
\begin{aligned}
b_{\alpha}(\nu) & =|\alpha|^{2} i \nu c_{\alpha}(\nu) \\
& =|\alpha|^{2} \frac{\Gamma\left(\frac{\langle\alpha, \rho\rangle}{\langle\alpha, \alpha\rangle}+\frac{1}{2} m_{\alpha}\right)}{\Gamma\left(\frac{\langle\alpha, \rho\rangle}{\langle\alpha, \alpha\rangle}\right)} \frac{\Gamma\left(\frac{1}{2} \frac{\langle\alpha, \rho\rangle}{\langle\alpha, \alpha\rangle}+\frac{1}{4} m_{\alpha}+\frac{1}{2} m_{2 \alpha}\right)}{\Gamma\left(\frac{1}{2} \frac{\langle\alpha, \rho\rangle}{\langle\alpha, \alpha\rangle}+\frac{1}{4} m_{\alpha}\right)} \frac{\Gamma(i \nu+1)}{\Gamma\left(i \nu+\frac{1}{2} m_{\alpha}\right)} \frac{\Gamma\left(\frac{i}{2} \nu+\frac{1}{4} m_{\alpha}\right)}{\Gamma\left(\frac{i}{2} \nu+\frac{1}{4} m_{\alpha}+\frac{1}{2} m_{2 \alpha}\right)} .
\end{aligned}
$$

Notice that $b_{\alpha}(-i \nu)^{ \pm 1}$ is a holomorphic function for $\operatorname{Im} \nu>-1 / 2$, with

$$
\frac{\Gamma(i \nu+1)}{\Gamma\left(i \nu+\frac{1}{2} m_{\alpha}\right)} \frac{\Gamma\left(\frac{i}{2} \nu+\frac{1}{4} m_{\alpha}\right)}{\Gamma\left(\frac{i}{2} \nu+\frac{1}{4} m_{\alpha}+\frac{1}{2} m_{2 \alpha}\right)} \sim 2^{\frac{m_{2 \alpha}}{2}} \nu^{1-\frac{m_{\alpha}}{2}-\frac{m_{2 \alpha}}{2}} \quad \text { as } \quad|\nu| \rightarrow+\infty .
$$

(The symbol $\sim$ means precisely that $\frac{\Gamma(i \nu+1)}{\Gamma\left(i \nu+\frac{1}{2} m_{\alpha}\right)} \frac{\Gamma\left(\frac{i}{2} \nu+\frac{1}{4} m_{\alpha}\right)}{\Gamma\left(\frac{i}{2} \nu+\frac{1}{4} m_{\alpha}+\frac{1}{2} m_{2 \alpha}\right)} \nu^{\frac{m_{\alpha}}{2}+\frac{m_{2 \alpha}}{2}-1}$ $\rightarrow 2^{\frac{m_{2 \alpha}}{2}}$ as $|\nu| \rightarrow+\infty$.) Hence $\mathbf{b}(-\lambda)^{ \pm 1}$ is a holomorphic function for 
$\lambda \in \mathfrak{a}+i \overline{\mathfrak{a}^{+}}$(actually in a neighborhood of $\mathfrak{a}+i \overline{\mathfrak{a}^{+}}$defined by $\omega(\operatorname{Im} \lambda)=$ $\min _{\alpha \in \Sigma^{+++}}\langle\alpha, \operatorname{Im} \lambda\rangle>-\eta$ for some small $\eta>0$ ), which has the following behavior:

$$
|\mathbf{b}(-\lambda)|^{ \pm 1} \asymp \prod_{\alpha \in \Sigma^{++}}(1+|\langle\alpha, \lambda\rangle|)^{ \pm 1 \mp m_{\alpha}+m_{2 \alpha} / 2}
$$

and whose derivatives can be estimated as follows, using Cauchy's formula:

$$
p\left(\frac{\partial}{\partial \lambda}\right) \mathbf{b}(-\lambda)^{ \pm 1}=\mathrm{O}\left(|\mathbf{b}(-\lambda)|^{ \pm 1}\right) .
$$

For an effective use of the inversion formula (2.2.2.ii), one needs precise information about spherical functions. Their behavior away from the walls is well described by the following converging expansion of Harish-Chandra (E) Gangolli).

Theorem 2.2.7. We have a converging expansion

$$
\varphi_{\lambda}(\exp H)=e^{-\langle\varrho, H\rangle} \sum_{q \in 2 Q} e^{-\langle q, H\rangle} \sum_{w \in W} \mathbf{c}(w \cdot \lambda) \gamma_{q}(w \cdot \lambda) e^{i\langle w \cdot \lambda, H\rangle}
$$

for all $\lambda \in \mathfrak{a}$ regular and $H \in \mathfrak{a}^{+}$. Here,

(i) $Q=\sum_{\alpha \in \Sigma^{++}} \mathbb{N} \alpha$ is the positive root lattice,

(ii) the leading coefficient $\gamma_{0}$ is equal to 1 ,

(iii) the other coefficients $\gamma_{q}(\lambda)$ are rational functions in $\lambda \in \mathfrak{a}_{\mathbb{C}}$, which have no poles for $\lambda \in \mathfrak{a}+i \overline{\mathfrak{a}^{+}}$(actually in a neighborhood of $\mathfrak{a}+i \overline{\mathfrak{a}^{+}}$ defined by $\omega(\operatorname{Im} \lambda)>-\eta$ for some small $\eta>0)$ and satisfy there

$$
\left|\gamma_{q}(\lambda)\right| \leq C(1+|q|)^{d}
$$

for some nonnegative constants $C$ and $d$ (independent of $q \in Q$ and $\left.\lambda \in \mathfrak{a}+i \overline{\mathfrak{a}^{+}}\right)$.

Moreover,

(iv) all derivatives of $\varphi_{\lambda}(\exp H)$ in $H$ have corresponding expansions

$$
\begin{aligned}
& p\left(\frac{\partial}{\partial H}\right) \varphi_{\lambda}(\exp H) \\
& =e^{-\langle\varrho, H\rangle} \sum_{q \in 2 Q} e^{-\langle q, H\rangle} \sum_{w \in W} \mathbf{c}(w \cdot \lambda) \gamma_{q}(w \cdot \lambda) p(i w \cdot \lambda-\varrho-q) e^{i\langle w \cdot \lambda, H\rangle} .
\end{aligned}
$$

Theorem 2.2.7 is proved for instance in [GV, Ch. 4] or [He2, Ch. IV]. The crucial point is the estimate in (iii), which is due to Gangolli and which provides quite a good control of convergence.

As far as the behavior of spherical functions along faces is concerned, the best information available is provided by the asymptotic expansion of Trombi \& Varadarajan. Before stating this result, let us introduce the height in $Q$ :

$$
\varkappa(q)=\sum_{\alpha \in \Sigma^{+++}} q_{\alpha} \quad \text { if } \quad q=\sum_{\alpha \in \Sigma^{+++}} q_{\alpha} \alpha .
$$


Moreover, given a subset $F$ in $\Sigma^{+++}$, let us decompose

$$
Q=Q_{F}+Q^{F} \quad \text { and }
$$

$$
\mathbf{c}(\lambda)=\mathbf{c}_{F}(\lambda) \mathbf{c}^{F}(\lambda), \quad \boldsymbol{\pi}(\lambda)=\boldsymbol{\pi}_{F}(\lambda) \boldsymbol{\pi}^{F}(\lambda), \quad \mathbf{b}(\lambda)=\mathbf{b}_{F}(\lambda) \mathbf{b}^{F}(\lambda),
$$

where $Q_{F}=\sum_{\alpha \in F} \mathbb{N} \alpha$ [resp. $Q^{F}=\sum_{\alpha \in \Sigma^{+++} \backslash F} \mathbb{N} \alpha$ ], and $\mathbf{c}_{F}(\lambda)$ [resp. $\left.\mathbf{c}^{F}(\lambda)\right], \boldsymbol{\pi}_{F}(\lambda)\left[\right.$ resp. $\left.\boldsymbol{\pi}^{F}(\lambda)\right], \mathbf{b}_{F}(\lambda)\left[\right.$ resp. $\left.\mathbf{b}^{F}(\lambda)\right]$ denote the products over $\alpha \in \Sigma_{F}^{++}\left[\right.$resp. over $\left.\alpha \in \Sigma^{++} \backslash \Sigma_{F}^{++}\right]$of $c_{\alpha}(\langle\alpha, \lambda\rangle /\langle\alpha, \alpha\rangle),\langle\alpha, \lambda\rangle$, $b_{\alpha}(\langle\alpha, \lambda\rangle /\langle\alpha, \alpha\rangle)$. Finally let

$$
\omega^{F}(H)=\min _{\alpha \in \Sigma^{++} \backslash F}\langle\alpha, H\rangle \quad \forall H \in \mathfrak{a} .
$$

Theorem 2.2.8. Let $F$ be a nontrivial subset of $\Sigma^{+++}$. Then we have an asymptotic expansion

$$
\varphi_{\lambda}(\exp H) \sim e^{-\left\langle Q^{F}, H\right\rangle} \sum_{q \in 2 Q^{F}} \sum_{w \in W_{F} \backslash W} \mathbf{c}^{F}(w \cdot \lambda) \varphi_{w \cdot \lambda, q}^{F}(\exp H)
$$

for all $\lambda \in \mathfrak{a}$ regular and $H \in \overline{\mathfrak{a}^{+}}$with $\omega^{F}(H)>0$. Specifically:

(i) $\varphi_{\lambda, 0}^{F}(y \exp H)=\varphi_{\lambda_{F}}^{F}(y) e^{i\left\langle\lambda^{F}, H\right\rangle}$ is the spherical function of index $\lambda$ on $M^{F}=M_{F} \exp \mathfrak{a}^{F}$.

(ii) The other terms $\varphi_{\lambda, q}^{F}(x)$ are bi- $K_{F}$-invariant $C^{\infty}$ functions in the variable $x \in M^{F}$ and $W_{F}$-invariant holomorphic functions in the variable $\lambda \in \mathfrak{a}+i \overline{\left(\mathfrak{a}^{F}\right)^{+}}$(actually $\lambda$ can be taken in a neighborhood of $\mathfrak{a}+i \overline{\left(\mathfrak{a}^{F}\right)^{+}}$defined by $\left|\operatorname{Im} \lambda_{F}\right|<\eta$ and $\omega^{F}\left(\operatorname{Im} \lambda^{F}\right)>-\eta$ for some small $\eta>0)$, which satisfy

$$
\varphi_{\lambda, q}^{F}(x)=\varphi_{\lambda, q}^{F}(y) e^{\langle i \lambda-q, H\rangle} \quad \forall x=y \exp H \in M^{F}=M_{F} \exp \mathfrak{a}^{F} .
$$

(iii) For every $q \in Q^{F}$ and $D \in \mathrm{U}\left(\mathfrak{m}^{F}\right)$, there exist a constant $d \geq 0$ and, for every $\eta>0$, another constant $C \geq 0$ such that

$$
\left|\varphi_{\lambda, q}^{F}(\exp H: D)\right| \leq C e^{\eta\left|H_{F}\right|}(1+|\lambda|)^{d} e^{-\left\langle\operatorname{Im} \lambda+\varrho_{F}+q, H\right\rangle}
$$

for all $\lambda \in \mathfrak{a}+i \overline{\left(\mathfrak{a}^{F}\right)^{+}}$and $H \in \overline{\mathfrak{a}^{+}}$.

(iv) For every integer $N>0$ and every $D \in \mathrm{U}\left(\mathfrak{m}^{F}\right)$, there exist a constant $d \geq 0$ and, for every $\eta>0$, another constant $C \geq 0$ such that

$$
\begin{gathered}
\left|\varphi_{\lambda}(\exp H: D)-e^{-\left\langle\varrho^{F}, H\right\rangle} \sum_{\substack{q \in 2 Q^{F} \\
\varkappa(q)<N}} \sum_{w \in W_{F} \backslash W} \mathbf{c}^{F}(w \cdot \lambda) \varphi_{w \cdot \lambda, q}^{F}\left(\exp H: e^{\varrho^{F}} \circ D \circ e^{-\varrho^{F}}\right)\right| \\
\leq C(1+|\lambda|)^{d}(1+|H|)^{d} e^{-\langle\varrho, H\rangle-N \omega^{F}(H)}
\end{gathered}
$$

for all $\lambda \in \mathfrak{a}$ regular and $H \in \overline{\mathfrak{a}^{+}}$with $\omega^{F}(H)>\eta$.

REMARK 2.2.9. (i) This result refines Harish-Chandra's constant term theory (see for instance [GV, Ch. 5]) by expanding spherical functions along a face beyond the leading term. 
(ii) Here is the relation between the expansions in Theorem 2.2.7 and in Theorem 2.2.8:

$$
\varphi_{\lambda, q}^{F}(\exp H)=e^{-\left\langle\varrho_{F}, H\right\rangle} \sum_{q^{\prime} \in 2 Q_{F}} e^{-\left\langle q+q^{\prime}, H\right\rangle} \sum_{w \in W_{F}} \mathbf{c}_{F}(w \cdot \lambda) \gamma_{q+q^{\prime}}(w \cdot \lambda) e^{i\langle w \cdot \lambda, H\rangle}
$$

for all $\lambda \in \mathfrak{a}\left(\Sigma_{F^{-}}\right)$regular and $H \in \mathfrak{a}^{+}$.

(iii) Expansions along faces actually converge [CMi], like expansions away from the walls. But we miss a control comparable to the Gangolli estimates in Theorem 2.2.7.iii.

Theorem 2.2.8 is essentially a restatement adapted to our needs of the main results in $[\mathrm{TrV}]$, which are reproduced in $[\mathrm{GV}, \mathrm{Ch} .7]$. Let us elaborate on (ii) and (iii).

Consider the algebra $\mathcal{A}^{F}$ of functions on $M^{F}$, which is generated by 1 and by the matrix entries of $a^{F}(x)=\operatorname{Ad}_{\mathfrak{n}^{F}}\left\{x^{-1} \theta(x)\right\}$, and which is graded by $\mathcal{A}^{F}=\bigoplus_{q \in Q^{F}} \mathcal{A}_{q}^{F}$, where $\mathcal{A}_{q}^{F}=\left\{f \in \mathcal{A}^{F} \mid f(x \exp H)=f(x) e^{-\langle q, H\rangle}\right.$, $\left.\forall x \in M^{F}, \forall H \in \mathfrak{a}^{F}\right\}$. We know from [loc. cit.] that $P_{q}(\lambda) \varphi_{\lambda, q}^{F}$ belongs to $\left\{\mathcal{A}_{q}^{F} \otimes \mathrm{U}\left(\mathfrak{m}^{F}\right)\right\}^{K_{F}} \varphi_{\lambda}^{F}$, where $P_{q}$ is a $W_{F}$-invariant polynomial and $\mathrm{U}\left(\mathfrak{m}^{F}\right)$ acts on $\varphi_{\lambda}^{F}$ by differentiation on the right. Hence $P_{q}(\lambda) \varphi_{\lambda, q}^{F}(x)$ is a $W_{F^{-}}$ invariant holomorphic function in $\lambda \in \mathfrak{a}_{\mathbb{C}}$ and a bi- $K_{F}$-invariant $C^{\infty}$ function in $x \in M^{F}$, which has the required homogeneity

$$
P_{q}(\lambda) \varphi_{\lambda, q}^{F}(x \exp H)=P_{q}(\lambda) \varphi_{\lambda, q}^{F}(x) e^{\langle i \lambda-q, H\rangle} \quad \forall x \in M_{F}, \forall H \in \mathfrak{a}^{F},
$$

and which can be estimated by

$$
\left|P_{q}(\lambda) \varphi_{\lambda, q}^{F}(\exp H)\right| \leq C_{q}(1+|\lambda|)^{d_{q}}\left(1+\left|H_{F}\right|\right)^{d} e^{\left|\operatorname{Im} \lambda_{F}\right|\left|H_{F}\right|-\left\langle\operatorname{Im} \lambda^{F}+\varrho_{F}+q, H\right\rangle}
$$

for $H \in \overline{\mathfrak{a}^{+}}\left(C_{q}, d_{q}\right.$ and $d$ are nonnegative constants, the first two depending on $q)$. Moreover similar estimates hold for all derivatives $P_{q}(\lambda) \varphi_{\lambda, q}^{F}(\exp H: D)$ with respect to $D \in \mathrm{U}\left(\mathfrak{m}^{F}\right)$, since $\mathrm{U}\left(\mathfrak{m}^{F}\right)$ preserves $\mathcal{A}_{q}^{F}$. Thus the main problem consists of getting rid of the factor $P_{q}(\lambda)$. Since $P_{q}(\lambda)$ is a product of (non necessarily distinct) factors $f(\lambda)=\langle\mu, \lambda\rangle+i \nu$, with $\mu \in \mathfrak{a} \backslash\{0\}$ and $\nu \in \mathbb{R} \backslash\{0\}$, this will be achieved by repeated application of the following elementary lemma.

LEMmA 2.2.11. Let $U$ be an open subset in $\mathbb{R}^{\ell}, T(U)=U+i \mathbb{R}^{\ell}$ the open tube over $U$ in $\mathbb{C}^{\ell}, \Xi$ a $C^{\infty}$ manifold, $F=F(z ; \xi)$ a holomorphic function in $z \in T(U)$ depending smoothly on $\xi \in \Xi$, and $f(z)=\langle\mu, z\rangle+\nu$ an affine function on $\mathbb{C}^{\ell}$ with $\mu \in \mathbb{R}^{\ell} \backslash\{0\}$.

(i) Assume that $F(z ; \xi)$ vanishes whenever $f(z)$ does (in $T(U)$ ). Then $G(z ; \xi)=F(z ; \xi) / f(z)$ is a holomorphic function in $z \in T(U)$ depending smoothly on $\xi \in \Xi$. 
(ii) Moreover, for any $\eta>0$, there exists a nonnegative constant $C$ such that

$$
|G(z ; \xi)| \leq C \sup _{|\zeta-z| \leq \eta}|F(\zeta ; \xi)|
$$

for all $z \in T(U)$ at distance $>\eta$ from $\mathbb{C}^{\ell} \backslash T(U)$ and for all $\xi \in \Xi$.

Proof of Lemma 2.2.11. One can reduce to the case $f(z)=z_{1}$.

(i) Let $\zeta \in T(U)$. If $\zeta_{1} \neq 0$, then $G(z ; \xi)=F(z ; \xi) / z_{1}$ defines obviously a holomorphic function in $z$, for $z$ close to $\zeta$, which depends smoothly in $\xi$. If $\zeta_{1}=0$, we have

$$
\begin{aligned}
F(z ; \xi) & =F\left(0, z_{2}, \ldots, z_{\ell} ; \xi\right)+\int_{0}^{1} d t \frac{\partial}{\partial t} F\left(t z_{1}, z_{2}, \ldots, z_{\ell} ; \xi\right) \\
& =z_{1} \int_{0}^{1} d t\left(\partial_{1} F\right)\left(t z_{1}, z_{2}, \ldots, z_{\ell} ; \xi\right)
\end{aligned}
$$

for $z$ close to $\zeta$, hence also in this case

$$
G(z ; \xi)=\frac{F(z ; \xi)}{z_{1}}=\int_{0}^{1} d t\left(\partial_{1} F\right)\left(t z_{1}, z_{2}, \ldots, z_{\ell} ; \xi\right)
$$

is holomorphic in $z$ and $C^{\infty}$ in $\xi$.

(ii) Let $z \in T(U)$. If $\left|z_{1}\right| \geq \frac{\eta}{3}$, then $|G(z ; \xi)| \leq \frac{3}{\eta}|F(z ; \xi)|$. Otherwise

$$
G(z ; \xi)=\frac{1}{2 \pi i} \int_{\left|\zeta_{1}\right|=2 \eta / 3} d \zeta_{1} \int_{0}^{1} d t \frac{F\left(\zeta_{1}, z_{2}, \ldots, z_{\ell} ; \xi\right)}{\left(\zeta_{1}-t z_{1}\right)^{2}}
$$

by Cauchy's formula, hence $|G(z ; \xi)| \leq \frac{3}{\eta} \sup _{|\zeta-z| \leq \eta}|F(\zeta ; \xi)|$.

This concludes the proof of Lemma 2.2.11.

Let us now complete the proof of (ii) and (iii) in Theorem 2.2.8. Restrict temporarily to $H \in \mathfrak{a}^{+}$. We know (see Remark 2.2.9) that $\lambda \mapsto$ $\boldsymbol{\pi}_{F}(\lambda) \varphi_{\lambda, q}^{F}(\exp H)$ is a holomorphic function in a tubular neighborhood $T_{\eta}=\left\{\lambda \in \mathfrak{a}_{\mathbb{C}}|| \operatorname{Im} \lambda_{F} \mid<\eta\right.$ and $\left.\omega^{F}\left(\operatorname{Im} \lambda^{F}\right)>-\eta\right\}$ of $\mathfrak{a}+i \overline{\left(\mathfrak{a}^{F}\right)^{+}}$ in $\mathfrak{a}_{\mathbb{C}}$. Since $\boldsymbol{\pi}_{F}(\lambda)$ and $P_{q}(\lambda)$ have no common factors, we deduce that $P_{q}(\lambda) \varphi_{\lambda, q}^{F}(\exp H)$ vanishes in $T_{\eta}$ whenever $P_{q}(\lambda)$ does. By combining this observation with Lemma 2.2.11.i, we can eliminate successively all factors in $P_{q}(\lambda)$ and obtain as a first conclusion that $\varphi_{\lambda, q}^{F}(x)$ is holomorphic in $\lambda \in T_{\eta}$ and $C^{\infty}$ in $x \in \exp \mathfrak{a}^{+}$. This result extends to $x \in K_{F} \exp \left\{\left(\mathfrak{a}^{F}\right)^{+}+\mathfrak{a}^{F}\right\} K_{F}$ by bi- $K_{F}$-invariance and $\left(\exp \mathfrak{a}^{F}\right)$-homogeneity. Further extension to $x \in M^{F}$ is achieved by density and by reapplying Lemma 2.2.11.i to $P_{q}(\lambda) \varphi_{\lambda, q}^{F}(x)$. Finally the estimate (iii) in Theorem 2.2 .8 is obtained by applying Lemma 2.2.11.ii to the expression $P_{q}(\lambda) \varphi_{\lambda, q}^{F}(\exp H: D) e^{-i\left\langle\lambda^{F}, H\right\rangle}$ and using (2.2.10) for derivatives of $\varphi_{\lambda, q}^{F}$. 
Finally let us recall the particular behavior of the basic spherical function $\varphi_{0}$.

Proposition 2.2.12. (i) Global estimate:

$$
\varphi_{0}(\exp H) \asymp\left\{\prod_{\alpha \in \Sigma^{++}}(1+\langle\alpha, H\rangle)\right\} e^{-\langle\varrho, H\rangle} \quad \forall H \in \overline{\mathfrak{a}^{+}} .
$$

(The symbol $\asymp$, let us recall, means precisely that there exist two constants $0<C_{1} \leq C_{2}<+\infty$ such that $\left.C_{1} \leq \frac{\varphi_{0}(\exp H)}{\left\{\prod_{\alpha \in \Sigma^{++}}(1+\langle\alpha, H\rangle)\right\} e^{-\langle\varrho, H\rangle}} \leq C_{2} \forall H \in \overline{\mathfrak{a}^{+}}\right)$.

(ii) Asymptotics:

$$
\begin{gathered}
\varphi_{0}(\exp H) \sim c_{3}^{F} \boldsymbol{\pi}^{F}(H) \varphi_{0}^{F}(\exp H) e^{-\left\langle\varrho^{F}, H\right\rangle} \\
\text { when }\left\{\begin{array}{l}
H \in \overline{\mathfrak{a}^{+}}, \\
\langle\alpha, H\rangle=\mathrm{o}\left(\omega^{F}(H)\right) \quad \forall \alpha \in F, \\
\omega^{F}(H)=\min _{\alpha \in \Sigma^{+++} \backslash F}\langle\alpha, H\rangle \rightarrow+\infty .
\end{array}\right.
\end{gathered}
$$

(The symbol $\sim$, let us recall, means precisely that $\frac{\varphi_{0}(\exp H)}{\pi^{F}(H) \varphi_{0}^{F}(\exp H) e^{-\left\langle\varrho^{F}, H\right\rangle}} \rightarrow$ $c_{3}^{F}$ under the indicated assumptions.) Here $F$ is a proper subset of $\Sigma^{+++}$ (possibly empty) and $c_{3}^{F}=\boldsymbol{\pi}^{F}(\widetilde{\varrho})^{-1} \mathbf{b}^{F}(0)$ a positive constant, with $\widetilde{\varrho}=$ $\frac{1}{2} \sum_{\alpha \in \Sigma^{++}} \alpha$.

REMARK 2.2.13. (i) Recall that $\varphi_{0}$ controls all spherical functions $\varphi_{\lambda}$ with parameter $\lambda \in \mathfrak{a}$. More precisely, for every $D \in \mathrm{U}(\mathfrak{g})$,

$$
\varphi_{\lambda}(x: D)=\mathrm{O}\left((1+|\lambda|)^{\operatorname{deg} D} \varphi_{0}(x)\right) \quad \forall \lambda \in \mathfrak{a}, \forall x \in G .
$$

This follows easily from (2.2.1) (see for instance [GV, Proposition 4.6.2]).

(ii) We can disregard Euclidean factors in $\mathbb{X}=G / K$, which do not contribute to $\varphi_{0}$, and will do so in the rest of this subsection.

(iii) The asymptotics in Proposition 2.2.12.ii hold in particular when $H \in \overline{\mathfrak{a}^{+}}$tends to infinity in either of the following ways, which are most often considered:

$$
\begin{aligned}
& \left\{\begin{array}{l}
\langle\alpha, H\rangle \text { remains bounded } \forall \alpha \in F, \\
\langle\alpha, H\rangle \rightarrow+\infty \quad \forall \alpha \in \Sigma^{+++} \backslash F,
\end{array}\right. \\
& \left\{\begin{array}{l}
\langle\alpha, H\rangle=\mathrm{o}(|H|) \quad \forall \alpha \in F, \\
\langle\alpha, H\rangle \asymp|H| \quad \forall \alpha \in \Sigma^{+++} \backslash F .
\end{array}\right.
\end{aligned}
$$

These particular asymptotics, for the various functions $\varphi_{0}^{F}$, are actually equivalent to the general asymptotics stated in Proposition 2.2.12.ii . Let us for instance deduce the general case from the particular case (a). In the 
proof of Proposition 2.2.12.ii, we shall do the same with (b). Assume that, for all subsets $F_{1} \subset F_{2}$ in $\Sigma^{+++}$,

$$
\begin{gathered}
\varphi_{0}^{F_{2}}(\exp H) \sim\left\{\prod_{\alpha \in \Sigma_{F_{2}}^{++} \backslash \Sigma_{F_{1}}^{++}}\langle\alpha, \widetilde{\varrho}\rangle^{-1} \mathbf{b}_{\alpha}(0)\langle\alpha, H\rangle\right\} \varphi_{0}^{F_{1}}(\exp H) e^{-\left\langle\varrho_{F_{2}}-\varrho_{F_{1}}, H\right\rangle} \\
\text { when }\left\{\begin{array}{l}
H \in \overline{\mathfrak{a}_{F_{2}}^{+}}, \\
\langle\alpha, H\rangle \text { remains bounded } \forall \alpha \in F_{1}, \\
\langle\alpha, H\rangle \rightarrow+\infty, \quad \forall \alpha \in F_{2} \backslash F_{1},
\end{array}\right.
\end{gathered}
$$

but that the asymptotics in Proposition 2.2.12.ii fail to hold. Thus there exists a proper subset $F_{2}$ of $\Sigma^{+++}$and a sequence $H_{j} \in \overline{\mathfrak{a}^{+}}$such that

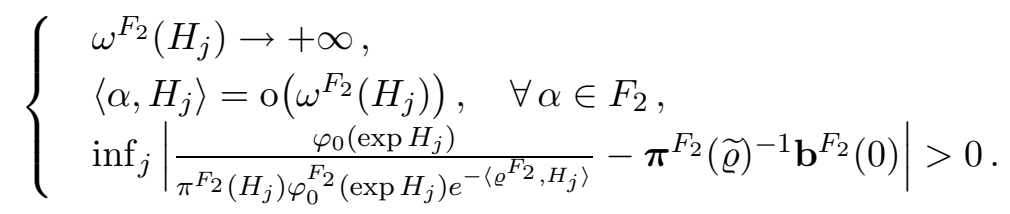

By passing to a subsequence, we can assume that

$$
\left\{\begin{array}{l}
\sup _{j}\left\langle\alpha, H_{j}\right\rangle<+\infty, \quad \forall \alpha \in F_{2}, \\
\left.\left\langle\alpha, H_{j}\right\rangle \rightarrow+\infty, \quad \forall \alpha \in F_{2} \backslash F_{1} \quad \text { (actually } \forall \alpha \in \Sigma^{+++} \backslash F_{1}\right),
\end{array}\right.
$$

for a subset $F_{1}$ of $F_{2}$. According to our assumptions,

$$
\frac{\boldsymbol{\pi}\left(H_{j}\right)^{-1} e^{\left\langle\varrho, H_{j}\right\rangle} \varphi_{0}\left(\exp H_{j}\right)}{\boldsymbol{\pi}_{F_{2}}\left(H_{j}\right)^{-1} e^{\left\langle\varrho_{F_{2}}, H_{j}\right\rangle} \varphi_{0}^{F_{2}}\left(\exp H_{j}\right)} \longrightarrow \frac{\boldsymbol{\pi}(\widetilde{\varrho})^{-1} \mathbf{b}(0)}{\boldsymbol{\pi}_{F_{2}}(\widetilde{\varrho})^{-1} \mathbf{b}_{F_{2}}(0)} .
$$

Hence a contradiction.

(iv) (See also Remark 5.2.2.iii.) The correct asymptotics

$$
\varphi_{0}(\exp H) \sim c_{F} \boldsymbol{\pi}^{F}(H) \varphi_{0}^{F}(\exp H) e^{-\left\langle\varrho^{F}, H\right\rangle}
$$

were announced in [Ol2] under the assumptions

$$
\left\{\begin{array}{l}
H \in \overline{\mathfrak{a}^{+}} \text {tends to infinity, i.e. }|H| \rightarrow+\infty, \\
\text { the } \mathfrak{a}_{F^{-}} \text {-component } H_{F} \text { tends to a vector in } \overline{\left(\mathfrak{a}_{F}\right)^{+}} .
\end{array}\right.
$$

But the analysis along faces relied on a misuse of Harish-Chandra's expansion (recalled in Theorem 2.2.7), as came out in the preprint version of [O13]. The final version contains the weaker result [O13, Proposition 2.6], where asymptotics of $\varphi_{0}(\exp H)$ are stated with a nonexplicit polynomial factor and under the additional assumptions

$$
\left\{\begin{array}{l}
\lim H_{F} \neq 0 \\
\frac{H}{|H|} \text { i.e. } \frac{H^{F}}{\left|H^{F}\right|} \text { tends to a unit vector in } \overline{\left(\mathfrak{a}^{F}\right)^{+}} .
\end{array}\right.
$$

But the proof requires actually more, namely $H \in \mathfrak{a}^{+}$and $\lim H_{F} \in\left(\mathfrak{a}_{F}\right)^{+}$. Besides, notice that error terms, involving derivatives of $f$, are missing in 
[O13, Lemma 2.5]. Thus the gap in Olshanetsky's results about $\varphi_{0}(\exp H)$ consists essentially in tangential asymptotics along faces.

Proof of Proposition 2.2.12. The global estimate (i) was established in [An1], essentially as a consequence of the Harish-Chandra converging expansion away from the walls (Theorem 2.2.7). The same expansion yields the asymptotics (ii) away from the walls, i.e. when $F=\emptyset$. We refer to the proof of $[\mathrm{GV}$, Theorem 4.6.6] for details. In addition we use the identity $\partial(\boldsymbol{\pi}) \boldsymbol{\pi}=|W| \boldsymbol{\pi}(\widetilde{\varrho})$, which is obtained by applying $\left.\boldsymbol{\pi}(\partial / \partial H)\right|_{H=0}$ to the Weyl denominator formula

$$
\prod_{\alpha \in \Sigma^{++}} 2 \sinh \frac{\langle\alpha, H\rangle}{2}=\sum_{w \in W}(\operatorname{det} w) e^{\langle w \cdot \widetilde{\varrho}, H\rangle}
$$

(see for instance [He2, Proposition I.5.15]).

Let us turn to the asymptotics (ii) along a face, i.e. relatively to a nontrivial subset $F$ of $\Sigma^{+++}$and let us first consider the special assumptions (2.2.13.iii.b). In this case we use the Trombi-Varadarajan asymptotic expansion along faces (Theorem 2.2.8), or the simpler Harish-Chandra constant term theory (see for instance [GV, Ch.5]), and more precisely the resulting asymptotics [GV, Theorem 5.9.5]

$$
\begin{aligned}
\varphi_{0}(\exp H)=e^{-\left\langle\varrho^{F}, H\right\rangle} p^{F}\left(-i \frac{\partial}{\partial \lambda}\right) & \left.\right|_{\lambda=0} \varphi_{\lambda}^{F}(\exp H) \\
& +\mathrm{O}\left((1+|H|)^{d} e^{-\langle\varrho, H\rangle-2 \omega^{F}(H)}\right),
\end{aligned}
$$

which holds for $H \in \overline{\mathfrak{a}^{+}}$with $\omega^{F}(H)$ bounded below. Here $p^{F}$ is a polynomial on $\mathfrak{a}$, which is $W$-harmonic and $W_{F}$-invariant, and which is uniquely determined by (2.2.15). Recall that $p^{\emptyset}=c_{3}^{\emptyset} \pi+$ derivatives of $\pi$ (see the proof of $[\mathrm{GV}$, Theorem 4.6.6]). We need a similar information about the other polynomials $p^{F}$. First of all, the space $\mathrm{H}_{W}(\mathfrak{a})^{W_{F}}$ of $W$-harmonic $W_{F}$-invariant polynomials on $\mathfrak{a}$ can be described as follows:

$$
\mathrm{H}_{W}(\mathfrak{a})^{W_{F}}=\partial\left(\mathrm{P}(\mathfrak{a})^{W_{F}}\right) \partial\left(\boldsymbol{\pi}_{F}\right) \boldsymbol{\pi}=\partial\left(\mathrm{H}_{W}(\mathfrak{a})^{W_{F}}\right) \partial\left(\boldsymbol{\pi}_{F}\right) \boldsymbol{\pi} .
$$

This is easily deduced from the case $F=\emptyset$, which is well-known (see for instance [He2, Theorem III.3.6.i]). Thus $\partial\left(\boldsymbol{\pi}_{F}\right) \boldsymbol{\pi}$ has maximal degree in $\mathrm{H}_{W}(\mathfrak{a})^{W_{F}}$ and all other elements are derivatives thereof. In particular

$$
p^{F}=c^{F} \partial\left(\boldsymbol{\pi}_{F}\right) \boldsymbol{\pi}+\partial\left(q^{F}\right) \partial\left(\boldsymbol{\pi}_{F}\right) \boldsymbol{\pi}=c^{F}\left\{\partial\left(\boldsymbol{\pi}_{F}\right) \boldsymbol{\pi}_{F}\right\} \boldsymbol{\pi}^{F}+\ldots,
$$

where $c^{F}$ is a constant, $q^{F}$ is a $W_{F}$-invariant polynomial on $\mathfrak{a}$ with no constant term, $\partial\left(\pi_{F}\right) \pi_{F}=\left|W_{F}\right| \pi_{F}\left(\widetilde{\varrho}_{F}\right)=\left|W_{F}\right| \pi_{F}(\widetilde{\varrho})$ is a positive constant, and the dots stand for a sum of terms obtained by suppressing some $\alpha$ in the product $\boldsymbol{\pi}^{F}=\prod_{\alpha \in \Sigma^{++} \backslash \Sigma_{F}^{++}} \alpha$ or by replacing them by some other 
$\alpha \in \Sigma_{F}^{++}$. Next, let us consider the expression

$\left.p^{F}\left(-i \frac{\partial}{\partial \lambda}\right)\right|_{\lambda=0} \varphi_{\lambda}^{F}(\exp H)=\int_{K_{F}} d k p^{F}\left(H\left\{\left(\exp H_{F}\right) k\right\}+H^{F}\right) e^{-\left\langle\varrho_{F}, H\left\{\left(\exp H_{F}\right) k\right\}\right\rangle}$, which is a consequence of Harish-Chandra's integral formula (2.2.1) for $\varphi_{\lambda}^{F}$. By expanding $p^{F}\left(H\left(\left(\exp H_{F}\right) k\right)+H^{F}\right)$ and estimating

$$
\left|H\left\{\left(\exp H_{F}\right) k\right\}\right| \leq\left|H_{F}\right| \asymp \max _{\alpha \in F}\langle\alpha, H\rangle=\mathrm{o}(|H|),
$$

we obtain

$$
\begin{aligned}
\left.p^{F}\left(-i \frac{\partial}{\partial \lambda}\right)\right|_{\lambda=0} \varphi_{\lambda}^{F}(x)=c^{F}\left|W_{F}\right| \pi_{F}(\widetilde{\varrho}) \pi^{F}\left(H^{F}\right) \varphi_{0}^{F}(\exp H) \\
+\mathrm{o}\left(|H|^{\left|\Sigma^{++}\right|-\left|\Sigma_{F}^{++}\right|} \varphi_{0}^{F}(\exp H)\right),
\end{aligned}
$$

hence

$$
\begin{aligned}
& \varphi_{0}(\exp H)=c^{F}\left|W_{F}\right| \pi_{F}(\widetilde{\varrho}) \pi^{F}(H) \varphi_{0}^{F}(\exp H) e^{-\left\langle\varrho^{F}, H\right\rangle} \\
&+\mathrm{o}\left(|H|^{\left|\Sigma^{++}\right|-\left|\Sigma_{F}^{++}\right|} \varphi_{0}^{F}(\exp H) e^{-\left\langle\varrho^{F}, H\right\rangle}\right) \\
& \sim c^{F}\left|W_{F}\right| \pi_{F}(\widetilde{\varrho}) \pi^{F}(H) \varphi_{0}^{F}(\exp H) e^{-\left\langle\varrho^{F}, H\right\rangle} .
\end{aligned}
$$

Finally the constant $c^{F}=\left|W_{F}\right|^{-1} \boldsymbol{\pi}(\widetilde{\varrho})^{-1} \mathbf{b}^{F}(0)$ is determined by considering a particular sequence $H_{j}$ going to infinity in $\overline{\mathfrak{a}^{+}}$with

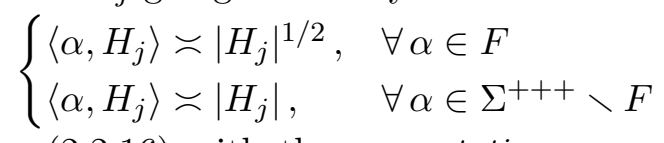

and by comparing (2.2.16) with the asymptotics away from the walls of both $\varphi_{0}\left(\exp H_{j}\right)$ and $\varphi_{0}^{F}\left(\exp H_{j}\right)$. Now that we have established the desired asymptotics in the special case (2.2.13.iii.b), let us extend our result to the general case. Arguing by contradiction, assume that there exist a subset $F_{0} \subsetneq \Sigma^{+++}$and a sequence $H_{j}^{(0)} \in \overline{\mathfrak{a}^{+}}$such that

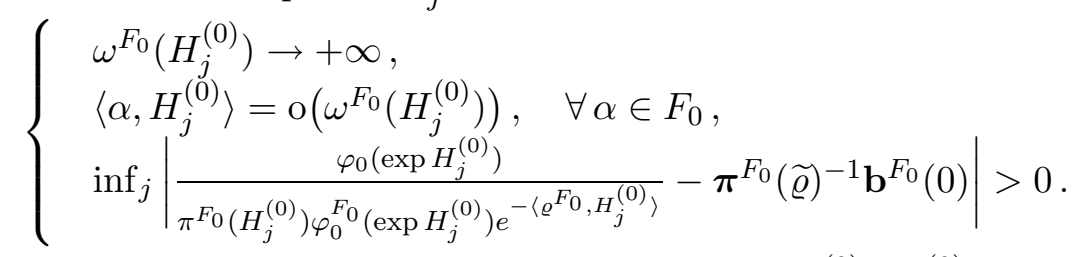

By passing to a subsequence, we can assume that $H_{j}^{(0)} /\left|H_{j}^{(0)}\right|$ tends to a unit vector $H_{\infty}^{(0)} \in \overline{\mathfrak{a}^{+}}$. Then $F_{1}=\left\{\alpha \in \Sigma^{+++} \mid\left\langle\alpha, H_{\infty}^{(0)}\right\rangle=0\right\}$ is a proper subset of $\Sigma^{+++}$containing $F_{0}$ and

$$
\varphi_{0}\left(\exp H_{j}^{(0)}\right) \sim \boldsymbol{\pi}^{F_{1}}(\widetilde{\varrho})^{-1} \mathbf{b}^{F_{1}}(0) \boldsymbol{\pi}^{F_{1}}\left(H_{j}^{(0)}\right) \varphi_{0}^{F_{1}}\left(\exp H_{j}^{(0)}\right) e^{-\left\langle\varrho^{\left.F_{1}, H_{j}^{(0)}\right\rangle},\right.}
$$

since

$$
\left\{\begin{aligned}
\left\langle\alpha, H_{j}^{(0)}\right\rangle=\mathrm{o}\left(\left|H_{j}^{(0)}\right|\right) & & \forall \alpha \in F_{1}, \\
\left\langle\alpha, H_{j}^{(0)}\right\rangle \asymp\left|H_{j}^{(0)}\right| & & \forall \alpha \in \Sigma^{+++} \backslash F_{1} .
\end{aligned}\right.
$$


If $F_{1}=F_{0}$, we have a contradiction. Otherwise $F_{1} \supsetneq F_{0}$ and in this case $H_{j}^{(1)}=\left(H_{j}\right)_{F_{1}}$ is a sequence in $\overline{\mathfrak{a}_{F_{1}}^{+}}$verifying

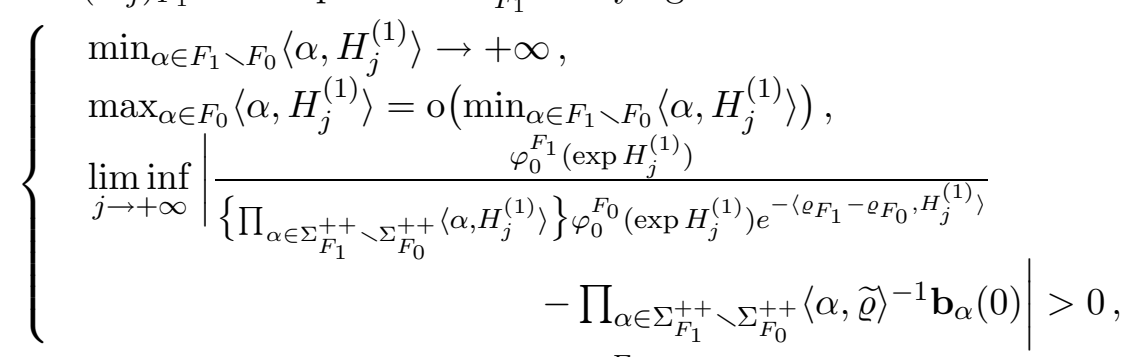

i.e. violating Proposition 2.2.12.ii for $\varphi_{0}^{F_{1}}$. Repeating the same reasoning, we end up with a contradiction, after a finite number of steps. This concludes the proof of Proposition 2.2.12.

\section{Heat Kernel Bounds}

During the last decades heat kernels have become a theme of extensive research in differential geometry, global analysis and probability. Among the vast literature we shall cite $[\mathrm{Ch}],[\mathrm{Da}],[\mathrm{Gr}],[\mathrm{R}],[\mathrm{Va}]$, [VaSCou] (and the bibliographies therein) as general references for the (scalar) heat kernel on Riemannian manifolds or Lie groups, and [An2,5], [CowGM1], [Lu] for the particular case of noncompact symmetric spaces.

It is well known that the heat kernel on $\mathbb{X}=G / K$ is given by

$$
h_{t}(x)=\frac{c_{2}}{|W|} \int_{\mathfrak{a}} \frac{d \lambda}{|\mathbf{c}(\lambda)|^{2}} e^{-t\left(|\lambda|^{2}+|\varrho|^{2}\right)} \varphi_{\lambda}(x)
$$

(see for instance the pioneer work $[\mathrm{G}]$ ). In [An2] the first author conjectured the global upper estimate

$$
\begin{array}{r}
h_{t}(\exp H) \leq C t^{-n / 2}\left\{\prod_{\alpha \in \Sigma^{++}}(1+\langle\alpha, H\rangle)(1+t+\langle\alpha, H\rangle)^{\frac{m_{\alpha}+m_{2 \alpha}}{2}-1}\right\} \\
\cdot e^{-|\varrho|^{2} t-\langle\varrho, H\rangle-\frac{|H|^{2}}{4 t}}
\end{array}
$$

for $t>0$ and $H \in \overline{\mathfrak{a}^{+}}$. This guess was based on some particular cases where specific expressions were available for the heat kernel, namely when $G$ is complex, when $\operatorname{rank} \mathbb{X}=1$, or when $G=\mathrm{SU}(p, q)$. Around the same time the global behavior of the heat kernel on real hyperbolic spaces was determined in $[\mathrm{DaM}]$ :

$$
h_{t}(r) \asymp t^{-\frac{n}{2}}(1+r)(1+t+r)^{\frac{n-3}{2}} e^{-\left(\frac{n-1}{2}\right)^{2} t-\frac{n-1}{2} r-\frac{r^{2}}{4 t}}
$$

for an appropriate normalization of the Riemannian structure, where $r$ denotes the geodesic distance to the origin. Thus the right hand side in (3.2) 
proved to be not only an upper bound but also a lower bound in this case. This result was extended later on to all hyperbolic spaces [GiMau], to the larger class of Damek-Ricci harmonic spaces [AnDY], and actually to a much wider family of radial Laplacians in [LorRo]. Let us turn to the higher rank case. In [An5] the first author obtained global upper bounds of the form

$$
t^{-d_{1}}(1+|H|)^{d_{2}} e^{-|\varrho|^{2} t-\langle\varrho, H\rangle-\frac{|H|^{2}}{4 t}},
$$

where $d_{1}$ and $d_{2}$ are positive constants depending on the position of $H \in \overline{\mathfrak{a}^{+}}$ with respect to the walls and on the relative size of $t>0$ and $1+|H|$. Although quite general and rather precise, this result was clearly not optimal. On the contrary (3.2) was established by specific computations for $G=\operatorname{SL}(n, \mathbb{R}), \operatorname{SL}(n, \mathbb{H})$ and "SL $(3, \mathbb{O})$ " in a series of papers by P. Sawyer ([Sa1-4]); moreover the right-hand side of (3.2) was shown in [Sa1] to be also a lower bound in the particular case $G=\mathrm{SL}(3, \mathbb{R})$. All these results has lead us to update (3.2) as follows.

Conjecture 3.4.

$h_{t}(\exp H) \asymp t^{-\frac{n}{2}}\left\{\prod_{\alpha \in \Sigma^{++}}(1+\langle\alpha, H\rangle)(1+t+\langle\alpha, H\rangle)^{\frac{m_{\alpha}+m_{2 \alpha}}{2}-1}\right\} e^{-|\varrho|^{2} t-\langle\varrho, H\rangle-\frac{|H|^{2}}{4 t}}$ for all $t>0$ and $H \in \overline{\mathfrak{a}^{+}}$.

REMARK 3.5. Let us make some comments about the various factors in this conjectural estimate. The whole expression reduces as expected to $t^{-n / 2} e^{-|H|^{2} / 4 t}$ in the Euclidean case or for small $t$ and $H$ in the general case. The exponential decay $e^{-|\varrho|^{2} t}$ in $t$ is connected with the bottom of the $L^{2}$ spectrum $\left[|\varrho|^{2},+\infty\right)$ of $-\Delta$, while the exponential decay $e^{-\langle\varrho, H\rangle}$ in $H$ is related both to the exponential growth of the volume (see (2.1.5)) and to the temperedness of the spherical functions $\varphi_{\lambda}$ entering (3.1) (see Remark 2.2.13.i). Eventually the expression between braces is related to the behavior of the c-function. More precisely, after dividing by $t^{m / 2}$,

$$
\prod_{\alpha \in \Sigma^{++}} \frac{1+\langle\alpha, H\rangle}{t}\left(1+\frac{1+\langle\alpha, H\rangle}{t}\right)^{\frac{m_{\alpha}+m_{2 \alpha}}{2}-1} \asymp \mathbf{c}\left(-i \frac{H_{1}+H}{\kappa_{1} t}\right)^{-1} \asymp\left|\mathbf{c}\left(-\frac{H_{2}+i H}{\kappa_{2} t}\right)\right|^{-1},
$$

where $H_{1}$ (resp. $\left.H_{2}\right)$ is any fixed element in $\mathfrak{a}^{+}$(resp. any fixed regular element in $\left.\mathfrak{a}+i \overline{\mathfrak{a}^{+}}\right)$and $\kappa_{1}, \kappa_{2}$ are any fixed positive constants.

Beside the heat kernel itself, we have also a conjectural estimate for its derivatives.

Conjecture 3.6. Let $D \in \mathrm{U}(\mathfrak{g})$. Then

$$
h_{t}(x: D)=\mathrm{O}\left(t^{-\operatorname{deg} D}(\sqrt{t}+t+|x|)^{\operatorname{deg} D} h_{t}(x)\right)
$$


for all $t>0$ and $x \in G$.

We shall establish these conjectures for general noncompact symmetric spaces but under some restrictions on the time and space variables, which prove fortunately to be unessential in all applications (see section 4).

Theorem 3.7. (i) Estimates away from the walls: Conjectures 3.4 and 3.6 hold when $\omega(H)=\min _{\alpha \in \Sigma^{+(++)}}\langle\alpha, H\rangle$ is large. This means precisely that there exist positive constants $\kappa, C_{1}, C_{2}$ and $C_{3}$ (the last one depending on $D$ ) such that

$$
C_{1} \leq \frac{h_{t}(\exp H)}{t^{-\frac{n}{2}}\left\{\prod_{\alpha \in \Sigma^{++}}\langle\alpha, H\rangle(t+\langle\alpha, H\rangle)^{\frac{m_{\alpha}+m_{2 \alpha}}{2}-1}\right\} e^{-|\varrho|^{2} t-\langle\varrho, H\rangle-\frac{|H|^{2}}{4 t}}} \leq C_{2}
$$

and

$$
\begin{aligned}
\left|h_{t}(\exp H: D)\right| \leq C_{3} t^{-\frac{n}{2}-\operatorname{deg} D}\left\{\prod_{\alpha \in \Sigma^{++}}\langle\alpha, H\rangle(t+\langle\alpha, H\rangle)^{\frac{m_{\alpha}+m_{2 \alpha}}{2}-1}\right\} \\
\times(t+|H|)^{\operatorname{deg} D} e^{-|\varrho|^{2} t-\langle\varrho, H\rangle-\frac{|H|^{2}}{4 t}}
\end{aligned}
$$

for all $t>0$ and $H \in \mathfrak{a}$ with $\omega(H) \geq \kappa$.

(ii) Global estimates: Conjectures 3.4 and 3.6 hold when $|H| \leq \kappa(1+t)$, $\kappa$ being an arbitrary positive constant. This means precisely that there exist positive constants $C_{1}, C_{2}$ and $C_{3}$ (depending on $\kappa$ and also on $D$ for $C_{3}$ ) such that

$$
C_{1} \leq \frac{h_{t}(\exp H)}{t^{-\frac{n}{2}}(1+t)^{\frac{m}{2}-\left|\Sigma^{++}\right|}\left\{\prod_{\alpha \in \Sigma^{++}}(1+\langle\alpha, H\rangle)\right\} e^{-|\varrho|^{2} t-\langle\varrho, H\rangle-\frac{|H|^{2}}{4 t}}} \leq C_{2}
$$

and

$$
\begin{aligned}
\left|h_{t}(\exp H: D)\right| \leq C_{3} t^{-\frac{n}{2}-\operatorname{deg} D}( & +t)^{\frac{m}{2}-\mid \Sigma^{++}} \mid\left\{\prod_{\alpha \in \Sigma^{++}}(1+\langle\alpha, H\rangle)\right\} \\
& \times(\sqrt{t}+t+|H|)^{\operatorname{deg} D} e^{-|\varrho|^{2} t-\langle\varrho, H\rangle-\frac{|H|^{2}}{4 t}}
\end{aligned}
$$

for all $t>0$ and $H \in \overline{\mathfrak{a}^{+}}$with $|H| \leq \kappa(1+t)$.

REMARK 3.8. Thus Conjectures 3.4 and 3.6 remain to be proved for $H \in \overline{\mathfrak{a}^{+}}$tending to infinity while staying at bounded distance to the walls and for $t \ll|H|$. Actually we shall obtain the upper estimate for all $t>0$ as long as $H$ does not tend to a wall. And we have anyway the upper bound (3.3) in the remaining cases.

The rest of this section will be devoted to the proof of Theorem 3.7, that we outline for the reader's convenience. Using classical results, we reduce in Step 0 to the semisimple setting and to $t+|H|$ large. After these 
preliminaries, we restate in Proposition 3.9 the upper estimates for the range $t \geq$ const. $(1+|H|)$, in a suitable form with regard to induction over the semisimple rank. Then we establish our estimates in eight successive steps, the upper ones in Steps 1-4 and the lower one in Steps 5-8. In both cases, we consider separately the ranges where

- $t \geq$ const. $\left(1+|H|^{2}\right)$,

- $t>0$ and $H$ stays away from the walls, i.e. $\omega(H) \geq$ const.,

- const. $\sqrt{t} \leq|H| \leq$ const. $t$ and $H$ is close to a face, i.e. $H \in \overline{\mathfrak{a}^{+}}(F, \delta, \varepsilon)$.

The lower estimate is more delicate than the upper ones and, in both cases, the main difficulty lies in the analysis along faces, which is performed in Steps 4,7 and 8.

Step 0: Preliminary reductions. (a) By specializing to $\mathbb{X}=G / K$ general heat kernel asymptotics (see for instance $[\mathrm{K}]$ ), we obtain all desirable information about $h_{t}(x)$ for $t$ small and $|x|$ bounded and can in particular check Conjectures 3.4 and 3.6 in this case. Since $h_{t}(x)$ is $C^{\infty}$ in $t \in(0,+\infty)$ and $x \in G$, and moreover positive, we can actually disregard in Theorem 3.7 any bounded range of $t$ and $H$.

(b) Let us get rid of a possible split component in $\mathbb{X}=G / K$. If $\mathbb{X}$ decomposes as $\mathbb{X}=\mathbb{X}^{\prime} \times \mathbb{X}^{\prime \prime}$, with $\mathbb{X}^{\prime}$ of noncompact type and $\mathbb{X}^{\prime \prime}$ Euclidean, then its heat kernel splits accordingly:

$$
h_{t}(\exp H)=h_{t}\left(\exp H^{\prime}\right) h_{t}\left(\exp H^{\prime \prime}\right) \quad \text { (obvious notation) }
$$

with $h_{t}\left(\exp H^{\prime \prime}\right)=(4 \pi t)^{-n / 2} e^{-\left|H^{\prime \prime}\right|^{2} / 4 t},|H|^{2}=\left|H^{\prime}\right|^{2}+\left|H^{\prime \prime}\right|^{2}$, and $\langle\alpha, H\rangle=$ $\left\langle\alpha, H^{\prime}\right\rangle$ for every $\alpha \in \Sigma$. This decomposition extends to derivatives, since $\mathrm{U}(\mathfrak{g})=\mathrm{U}\left(\mathfrak{g}^{\prime}\right) \otimes \mathrm{U}\left(\mathfrak{g}^{\prime \prime}\right)$ (obvious notation) and $h_{t}\left(\exp H: D^{\prime} D^{\prime \prime}\right)=h_{t}\left(\exp H^{\prime}: D^{\prime}\right) h_{t}\left(\exp H^{\prime \prime}: D^{\prime \prime}\right), \forall D^{\prime} \in \mathrm{U}\left(\mathfrak{g}^{\prime}\right), D^{\prime \prime} \in \mathrm{U}\left(\mathfrak{g}^{\prime \prime}\right)$. Consequently the estimates for $h_{t}(\exp H: D)$ in Theorem 3.7 (including $h_{t}(\exp H)$ itself) follow from the corresponding estimates for $h_{t}\left(\exp H^{\prime}: D^{\prime}\right)$.

The main difficulties in the proof of Theorem 3.7 lie in the estimates, especially the lower one, when $H$ tends to infinity along a face in $\overline{\mathfrak{a}^{+}}$. Our tools in this case will be the Trombi \& Varadarajan expansion for spherical functions (Theorem 2.2.8) and Harish-Chandra's reduction to lower rank symmetric spaces. This procedure requires to consider and estimate from above more general expressions, namely

$$
h_{\Omega ; t}(x)=\int_{\mathfrak{a}} \frac{d \lambda}{|\mathbf{c}(\lambda)|^{2}} \Omega(\lambda) e^{-t\left(|\varrho|^{2}+|\lambda|^{2}\right)} \varphi_{\lambda}(x)
$$

and their derivatives, where $\Omega(\lambda)$ are $W$-invariant functions which are holomorphic inside some tube $\left\{\lambda \in \mathfrak{a}_{\mathbb{C}}|| \operatorname{Im} \lambda \mid \leq \kappa\right\}$, which extend continuously 
to its boundary and which have (at most) polynomial growth. Let us denote by $\mathcal{O}_{\kappa}(\mathfrak{a})^{W}$ the space of all such functions $\Omega$, for any fixed $\kappa>0$. In the limit case $\kappa=0, \mathcal{O}_{0}(\mathfrak{a})^{W}$ consists simply of all $W$-invariant $C^{\infty}$ functions on $\mathfrak{a}$ with polynomial growth.

Proposition 3.9. Let $D \in \mathrm{U}(\mathfrak{g})$ and let $\kappa_{1}, \kappa_{2}, \eta$ be positive constants with $\eta<1$. Then there exists a nonnegative constant $C$ such that

$$
\begin{aligned}
\left|h_{\Omega ; t}(\exp H: D)\right| \leq & C\left\{\sup _{|\operatorname{Im} \lambda| \leq \kappa_{2}^{\prime}}|\Omega(\lambda)| e^{-\eta t|\operatorname{Re} \lambda|^{2}}\right\} \\
& \times t^{-\frac{\ell}{2}-\mid \Sigma^{++}}\left\{\prod_{\alpha \in \Sigma^{++}}(1+\langle\alpha, H\rangle)\right\} e^{-|\varrho|^{2} t-\langle\varrho, H\rangle-\frac{|H|^{2}}{4 t}}
\end{aligned}
$$

for every $\Omega \in \mathcal{O}_{\kappa_{2}^{\prime}}(\mathfrak{a})^{W}$ with $0 \leq \kappa_{2}^{\prime} \leq \kappa_{2}$ and for every $t \geq \kappa_{1}, H \in \overline{\mathfrak{a}^{+}}$ with $|H| \leq 2 \kappa_{2}^{\prime} t$.

We shall establish first the upper bounds in the easy cases, which were admittedly more or less known.

Step 1: Upper estimates for $t \geq$ const. $\left(1+|H|^{2}\right)$. Let $\Omega \in \mathcal{O}_{0}(\mathfrak{a})^{W}$, $D \in \mathrm{U}(\mathfrak{g}), \kappa>0$ and $0<\eta<1$. Using 2.2.5, Proposition 2.2.12.i and Remark 2.2.13.i, we estimate easily, for every $H \in \overline{\mathfrak{a}^{+}}$and $t \geq \kappa\left(1+|H|^{2}\right)$,

$$
\begin{aligned}
\left|h_{\Omega ; t}(\exp H: D)\right| \leq & C \int_{\mathfrak{a}} \frac{d \lambda}{|\mathbf{c}(\lambda)|^{2}}|\Omega(\lambda)| e^{-t\left(|\varrho|^{2}+|\lambda|^{2}\right)}\left|\varphi_{\lambda}(\exp H: D)\right| \\
\leq & C A_{t} e^{-|\varrho|^{2} t} \varphi_{0}(\exp H) \\
& \quad \times \int_{\mathfrak{a}} d \lambda \boldsymbol{\pi}(\lambda)^{2}(1+|\lambda|)^{m+\operatorname{deg} D} e^{-(1-\eta) t|\lambda|^{2}} \\
\leq & C A_{t} e^{-|\varrho|^{2} t}\left\{\prod_{\alpha \in \Sigma^{++}}(1+\langle\alpha, H\rangle)\right\} e^{-\langle\varrho, H\rangle} \\
& \quad \times t^{-\frac{\ell}{2}-\left|\Sigma^{++}\right|} \int_{\mathfrak{a}} d \lambda \boldsymbol{\pi}(\lambda)^{2}\left(1+\frac{|\lambda|}{\sqrt{t}}\right)^{m+\operatorname{deg} D} e^{-(1-\eta)|\lambda|^{2}} \\
\leq & C A_{t} t^{-\frac{\ell}{2}-\left|\Sigma^{++}\right|}\left\{\prod_{\alpha \in \Sigma^{++}}(1+\langle\alpha, H\rangle)\right\} e^{-|\varrho|^{2} t-\langle\varrho, H\rangle-\frac{|H|^{2}}{4 t}},
\end{aligned}
$$

where the dependence on $\Omega$ is entirely contained in $A_{t}=\sup _{\lambda \in \mathfrak{a}}|\Omega(\lambda)| e^{-\eta t|\lambda|^{2}}$. Notice that the Gaussian factor $e^{-|H|^{2} / 4 t}$ is trivial under the present assumption $t \geq \kappa|H|^{2}$.

Step 2: Upper estimates in Theorem 3.7.i. We shall restrict here to $t>0$ and $H \in \mathfrak{a}^{+}$with $\omega(H) \geq \kappa$ (this condition goes slightly beyond the actual statement of Theorem 3.7.i) where $\kappa$ is an arbitrary positive 
constant. Let $p \in \mathrm{P}(\mathfrak{a})$ and let us expand the spherical functions in

$$
p\left(\frac{\partial}{\partial H}\right) h_{t}(\exp H)=\frac{c_{2}}{|W|} \int_{\mathfrak{a}} \frac{d \lambda}{|\mathbf{c}(\lambda)|^{2}} e^{-t\left(|\varrho|^{2}+|\lambda|^{2}\right)} p\left(\frac{\partial}{\partial H}\right) \varphi_{\lambda}(\exp H)
$$

according to Theorem 2.2.7. Using the decompositions

$$
|\mathbf{c}(\lambda)|^{-2}=\mathbf{c}(w \cdot \lambda)^{-1} \mathbf{c}(-w \cdot \lambda)^{-1}, \quad \forall \lambda \in \mathfrak{a}, \forall w \in W
$$

and

we obtain

$$
\mathbf{c}(-\lambda)^{-1}=\boldsymbol{\pi}(-i \lambda) \mathbf{b}(-\lambda)^{-1}
$$

where

$$
p\left(\frac{\partial}{\partial H}\right) h_{t}(\exp H)=\sum_{q \in 2 Q} E_{q}
$$

$$
\begin{aligned}
E_{q}=c_{2} e^{-|\varrho|^{2} t-\langle\varrho+q, H\rangle} & \\
& \times \int_{\mathfrak{a}} d \lambda \boldsymbol{\pi}(-i \lambda) \mathbf{b}(-\lambda)^{-1} \gamma_{q}(\lambda) p(i \lambda-\varrho-q) e^{-t|\lambda|^{2}+i\langle\lambda, H\rangle} .
\end{aligned}
$$

Recall the remarkable and well-known cancellation of the remainder in

$$
\boldsymbol{\pi}\left(\frac{i}{2 t} \frac{\partial}{\partial \lambda}\right) e^{-t|\lambda|^{2}}=\boldsymbol{\pi}(-i \lambda) e^{-t|\lambda|^{2}}+\ldots
$$

which is due to the fact (see for instance [He2, Corollary III.3.8]) that there are no nonzero polynomials on $\mathfrak{a}$ which are skew symmetric under $W$ and of degree $<\left|\Sigma^{++}\right|$. Thus (3.10) becomes

$$
\begin{aligned}
& E_{q}=c_{2} 2^{-\left|\Sigma^{++}\right|} t^{-\left|\Sigma^{++}\right|} e^{-|\varrho|^{2} t-\langle\varrho+q, H\rangle} \\
& \times \sum_{\Sigma^{++}=\Sigma^{\prime} \sqcup \Sigma^{\prime \prime}} \int_{\mathfrak{a}} d \lambda e^{-t|\lambda|^{2}}\left\{\prod_{\alpha^{\prime} \in \Sigma^{\prime}}\left\langle\alpha^{\prime}, H\right\rangle\right\} e^{i\langle\lambda, H\rangle} \\
& \quad \times\left\{\prod_{\alpha^{\prime \prime} \in \Sigma^{\prime \prime}}\left(-i \partial_{\alpha^{\prime \prime}}\right)\right\}\left\{\mathbf{b}(-\lambda)^{-1} \gamma_{q}(\lambda) p(i \lambda-\varrho-q)\right\},
\end{aligned}
$$

after integrating by parts, and

$$
\begin{gathered}
E_{q}=c_{2} 2^{-\left|\Sigma^{++}\right|} t^{-\left|\Sigma^{++}\right|} e^{-|\varrho|^{2} t-\langle\varrho+q, H\rangle-\frac{|H|^{2}}{4 t}} \\
\times \sum_{\Sigma^{++}=\Sigma^{\prime} \sqcup \Sigma^{\prime \prime}}\left\{\prod_{\alpha^{\prime} \in \Sigma^{\prime}}\left\langle\alpha^{\prime}, H\right\rangle\right\} \int_{\mathfrak{a}} d \lambda e^{-t|\lambda|^{2}} \\
\times\left\{\prod_{\alpha^{\prime \prime} \in \Sigma^{\prime \prime}}\left(-i \partial_{\alpha^{\prime \prime}}\right)\right\}\left\{\mathbf{b}\left(-\lambda-i \frac{H}{2 t}\right)^{-1} \gamma_{q}\left(\lambda+i \frac{H}{2 t}\right) p\left(i \lambda-\frac{H}{2 t}-\varrho-q\right)\right\},
\end{gathered}
$$

after moving the contour of integration, which produces the expected Gaussian factor $e^{-|H|^{2} / 4 t}$. The last three factors can be estimated as follows:

$$
\begin{aligned}
& \left(\frac{\partial}{\partial \lambda}\right)^{\sigma} \mathbf{b}\left(-\lambda-i \frac{H}{2 t}\right)^{-1} \\
& \quad=\mathrm{O}\left(\left\{\prod_{\alpha \in \Sigma^{+}+}\left(1+\frac{\langle\alpha, H\rangle}{t}\right)^{\frac{m_{\alpha}+m_{2 \alpha}}{2}-1}\right\}(1+\sqrt{t}|\lambda|)^{\frac{m}{2}}\right),
\end{aligned}
$$




$$
\begin{aligned}
& \left(\frac{\partial}{\partial \lambda}\right)^{\sigma} \gamma_{q}\left(\lambda+i \frac{H}{2 t}\right)^{-1}=\mathrm{O}\left((1+|q|)^{d}\right) \\
& \left(\frac{\partial}{\partial \lambda}\right)^{\sigma} p\left(i \lambda-\frac{H}{2 t}-\varrho-q\right) \\
& \quad=\mathrm{O}\left((1+|q|)^{\operatorname{deg} p}\left(1+\frac{|H|}{t}\right)^{\operatorname{deg} p}(1+\sqrt{t}|\lambda|)^{\operatorname{deg} p}\right) .
\end{aligned}
$$

(3.12) follows from Theorem 2.2.8.iii and Cauchy's formula. (3.13) is obvious, except maybe for the elementary estimate

$$
|\lambda| \leq C\left(1+\frac{|H|}{t}\right)(1+\sqrt{t}|\lambda|) .
$$

(3.11) is obtained by combining (2.2.5), (2.2.6) and

$$
\frac{\langle\alpha, H\rangle}{2 t} \leq\left|\left\langle\alpha, \lambda+i \frac{H}{2 t}\right\rangle\right| \leq|\lambda|+\frac{\langle\alpha, H\rangle}{2 t} \leq C\left(1+\frac{\langle\alpha, H\rangle}{t}\right)(1+\sqrt{t}|\lambda|) .
$$

Since

we get finally

$$
\int_{\mathfrak{a}} d \lambda e^{-t|\lambda|^{2}}(1+\sqrt{t}|\lambda|)^{\frac{m}{2}+\operatorname{deg} p}=\mathrm{O}\left(t^{-\frac{\ell}{2}}\right),
$$

$$
\begin{aligned}
& \left|E_{q}\right| \leq C(1+|q|)^{d+\operatorname{deg} p} t^{-\frac{\ell}{2}}\left(1+\frac{|H|}{t}\right)^{\operatorname{deg} p}\left\{\prod_{\alpha \in \Sigma^{++}} \frac{\langle\alpha, H\rangle}{t}\left(1+\frac{\langle\alpha, H\rangle}{t}\right)^{\frac{m_{\alpha}+m_{2 \alpha}}{2}-1}\right\} \\
& \times e^{-|\varrho|^{2} t-\langle\varrho+q, H\rangle-\frac{|H|^{2}}{4 t}}
\end{aligned}
$$

and

$$
\begin{aligned}
\left|p\left(\frac{\partial}{\partial H}\right) h_{t}(\exp H)\right| \leq C t^{-\frac{\ell}{2}}\left(1+\frac{|H|}{t}\right)^{\operatorname{deg} p}\left\{\prod_{\alpha \in \Sigma^{++}} \frac{\langle\alpha, H\rangle}{t}\right. & \left.\left(1+\frac{\langle\alpha, H\rangle}{t}\right)^{\frac{m_{\alpha}+m_{2 \alpha}}{2}-1}\right\} \\
& \times e^{-|\varrho|^{2} t-\langle\varrho, H\rangle-\frac{|H|^{2}}{4 t}},
\end{aligned}
$$

after summing up over $q$. This estimate extends to all derivatives $h_{t}(\exp H: D)$ in a standard way. Recall indeed that, for every $D \in \mathrm{U}(\mathfrak{g})$, there exist polynomials $p_{1}, \ldots, p_{N} \in \mathrm{P}(\mathfrak{a})$ of degree $\leq \operatorname{deg} D$ and functions $a_{1}, \ldots, a_{N} \in C^{\infty}\left(\mathfrak{a}^{+}\right)$which are bounded away from the walls (i.e. when $\omega(H) \geq \kappa>0)$ such that

$$
f(\exp H: D)=\sum_{j=1}^{N} a_{j}(H) p_{j}\left(\frac{\partial}{\partial H}\right) f(\exp H) \quad \forall f \in C^{\infty}(G)^{\natural}, \forall H \in \mathfrak{a}^{+}
$$

(see for instance [GV, pp. 128-129]).

Step 3: Proof of Proposition 3.9 away from the walls. We shall restrict here to $t>0$ and $H \in \mathfrak{a}^{+}$with $\omega(H) \geq \kappa_{1}(1+\sqrt{t}$ ) (this condition goes again slightly beyond our actual needs for Proposition 3.9) and $|H| \leq 2 \kappa_{2}^{\prime} t$. Let us expand as in Step 2

$$
p\left(\frac{\partial}{\partial H}\right) h_{\Omega ; t}(\exp H)=\sum_{q \in 2 Q} E_{q}
$$


with

$$
\begin{aligned}
E_{q}=c_{2} e^{-|\varrho|^{2} t-\langle\varrho+q, H\rangle} & \\
& \times \int_{\mathfrak{a}} d \lambda \boldsymbol{\pi}(-i \lambda) \mathbf{b}(-\lambda)^{-1} \gamma_{q}(\lambda) p(i \lambda-\varrho-q) \Omega(\lambda) e^{-t|\lambda|^{2}+i\langle\lambda, H\rangle},
\end{aligned}
$$

but let us now handle these expressions differently in order to avoid differentiating $\Omega$. We move first the contour of integration and estimate next

$$
\begin{aligned}
& E_{q}=c_{2} e^{-|\varrho|^{2}} t-\langle\varrho+q, H\rangle-\frac{|H|^{2}}{4 t} \\
& \times \int_{\mathfrak{a}} d \lambda \boldsymbol{\pi}\left(\frac{H}{2 t}-i \lambda\right) \mathbf{b}\left(-\lambda-i \frac{H}{2 t}\right)^{-1} \gamma_{q}\left(\lambda+i \frac{H}{2 t}\right) \\
& \times p\left(i \lambda-\frac{H}{2 t}-\varrho-q\right) \Omega\left(\lambda+i \frac{H}{2 t}\right) e^{-t|\lambda|^{2}},
\end{aligned}
$$

using (3.10), (3.12), (3.13) (with $\sigma=0)$ and

$$
\boldsymbol{\pi}\left(\frac{H}{2 t}-i \lambda\right)=\mathrm{O}\left(\boldsymbol{\pi}\left(\frac{H}{t}\right)(1+\sqrt{t}|\lambda|)\right)
$$

which is obtained by expanding

$$
\boldsymbol{\pi}\left(\frac{H}{2 t}-i \lambda\right)=\prod_{\alpha \in \Sigma^{++}}\left\langle\alpha, \frac{H}{2 t}-i \frac{\sqrt{t} \lambda}{\sqrt{t}}\right\rangle
$$

and making use of the assumption $1 / \sqrt{t}=\mathrm{O}(\omega(H) / t)$. As a result

$$
\begin{aligned}
\left|E_{q}\right| \leq & C(1+|q|)^{d+\operatorname{deg} p}\left\{\sup _{|\operatorname{Im} \lambda| \leq \kappa_{2}^{\prime}}|\Omega(\lambda)| e^{-\eta t|\operatorname{Re} \lambda|^{2}}\right\} \\
& \times t^{-\frac{\ell}{2}}\left(1+\frac{|H|}{t}\right)^{\operatorname{deg} p}\left\{\prod_{\alpha \in \Sigma^{++}} \frac{\langle\alpha, H\rangle}{t}\left(1+\frac{\langle\alpha, H\rangle}{t}\right)^{\frac{m_{\alpha}+m_{2 \alpha}}{2}-1}\right\} \\
& \times e^{-|\varrho|^{2} t-\langle\varrho+q, H\rangle-\frac{|H|^{2}}{4 t}}
\end{aligned}
$$

We conclude as in Step 2.

As far as upper bounds are concerned, it remains for us to prove Proposition 3.9 along a face $\left(\mathfrak{a}^{F}\right)^{+}$, more precisely for $H \in \overline{\mathfrak{a}^{+}}(F, \delta, \varepsilon)$ with $\delta \ll \varepsilon$ and const. $\sqrt{t} \leq|H| \leq$ const.t. This will be achieved by induction on the semisimple rank. Thus let us assume that Proposition 3.9 holds for all proper symmetric subspaces with no Euclidean factor in $\mathbb{X}=G / K$.

Step 4: Upper estimates along faces. Let $\kappa_{1}, \kappa_{2}, \eta$ be positive constants with $\eta<1, \Omega \in \mathcal{O}_{\kappa_{2}^{\prime}}(\mathfrak{a})^{W}$ with $0 \leq \kappa_{2}^{\prime} \leq \kappa_{2}$, and $D \in \mathrm{U}(\mathfrak{g})$. We shall estimate

$$
h_{\Omega ; t}(\exp H: D)=\int_{\mathfrak{a}} \frac{d \lambda}{|\mathbf{c}(\lambda)|^{2}} \Omega(\lambda) e^{-t\left(|\varrho|^{2}+|\lambda|^{2}\right)} \varphi_{\lambda}(\exp H: D)
$$

for $t>0$ and $H \in \overline{\mathfrak{a}^{+}}(F, \delta, \varepsilon)$ with $\kappa_{1} \sqrt{t} \leq|H| \leq 2 \kappa_{2}^{\prime} t$. Here $F$ denotes any nontrivial subset of $\Sigma^{+++}$and $\varepsilon / \delta$ can be taken as large as we wish, 
according to Lemma 2.1.7.ii, let say $\varepsilon / \delta \geq\left(1+2 \kappa_{2}\right) c_{1}$, where $c_{1}$ is the constant occurring in Lemmas 2.1.6.ii and 2.1.7.i. Notice that all these assumptions imply that

$$
\begin{aligned}
& \text { t, } H \text { and } \omega^{F}(H) \text { are bounded below, } \\
& H_{F} \in \overline{\left(\mathfrak{a}_{F}\right)^{+}} \text {, } \\
& H^{F} \in\left(\mathfrak{a}^{F}\right)^{+} \text {, specifically } \omega^{F}\left(H^{F}\right) \geq \omega^{F}(H) \geq \varepsilon|H|, \\
& \left|H_{F}\right| \leq c_{1} \delta|H| \leq \varepsilon|H|, \\
& \frac{\left|H_{F}\right|^{2}}{t} \leq \frac{|H|}{t}\left|H_{F}\right| \leq 2 \kappa_{2} c_{1} \delta|H| \leq \varepsilon|H| .
\end{aligned}
$$

Let us restrict first to derivatives $D \in \mathrm{U}\left(\mathfrak{m}^{F}\right)=\mathrm{U}\left(\mathfrak{m}_{F}\right) \otimes \mathrm{U}\left(\mathfrak{a}^{F}\right)$, hence to $D=D_{F} \partial\left(p^{F}\right)$ with $D_{F} \in \mathrm{U}\left(\mathfrak{m}_{F}\right)$ and $p^{F} \in \mathrm{P}\left(\mathfrak{a}^{F}\right)$. By expanding the spherical functions in (3.15) according to Theorem 2.2 .8 with $N \geq 1+\frac{\kappa_{2}}{2 \varepsilon}$, we obtain

$$
h_{\Omega ; t}\left(\exp H: D_{F} \partial\left(p^{F}\right)\right)=\sum_{\substack{q \in 2 Q^{F} \\ \varkappa(q)<N}} E_{q}^{F}+R_{N}^{F}
$$

where

$$
\begin{aligned}
& E_{q}^{F}=\frac{c_{2}}{\left|W_{F}\right|} e^{-|\varrho|^{2} t-\left\langle\varrho^{F}, H\right\rangle} \\
\times & \int_{\mathfrak{a}} d \lambda\left|\mathbf{c}_{F}(\lambda)\right|^{-2} \mathbf{c}^{F}(-\lambda)^{-1} \Omega(\lambda) e^{-t|\lambda|^{2}} p^{F}\left(i \lambda^{F}-\varrho^{F}-q^{F}\right) \varphi_{\lambda, q}^{F}\left(\exp H: D_{F}\right)
\end{aligned}
$$

and $R_{N}^{F}$ denotes the remainder. $E_{0}^{F}$ is the main term, the most delicate to estimate, and the only one which does require the induction hypothesis. After decomposing the integral over $\mathfrak{a}=\mathfrak{a}_{F} \oplus \mathfrak{a}^{F}$ and moving the contour of integration, it can be rewritten

$$
\begin{aligned}
E_{0}^{F}= & \frac{c_{2}}{\left|W_{F}\right|} e^{-|\varrho|^{2} t-\left\langle\varrho^{F}, H\right\rangle-\frac{\left|H^{F}\right|^{2}}{4 t}} \\
& \times \int_{\mathfrak{a}_{F}} d \lambda_{F}\left|\mathbf{c}_{F}\left(\lambda_{F}\right)\right|^{-2} \Omega_{F ; t, H}\left(\lambda_{F}\right) e^{-t\left|\lambda_{F}\right|^{2}} \varphi_{\lambda_{F}}^{F}\left(\exp H_{F}: D_{F}\right),
\end{aligned}
$$

with

$$
\begin{aligned}
\Omega_{F ; t, H}\left(\lambda_{F}\right)= & \int_{\mathfrak{a}^{F}} d \lambda^{F} \boldsymbol{\pi}^{F}\left(\frac{H^{F}}{2 t}-i \lambda_{F}-i \lambda^{F}\right) \mathbf{b}^{F}\left(-\lambda_{F}-\lambda^{F}-i \frac{H^{F}}{2 t}\right)^{-1} \\
& \times p^{F}\left(i \lambda^{F}-\frac{H^{F}}{2 t}-\varrho^{F}\right) \Omega\left(\lambda_{F}+\lambda^{F}+i \frac{H^{F}}{2 t}\right) e^{-t\left|\lambda^{F}\right|^{2}} .
\end{aligned}
$$

We claim that $\Omega_{F ; t, H}$ belongs to $\mathcal{O}_{\kappa_{F}}\left(\mathfrak{a}_{F}\right)^{W_{F}}$ and that

$$
\sup _{\left|\operatorname{Im} \lambda_{F}\right| \leq \kappa_{F}}\left|\Omega_{F ; t, H}\left(\lambda_{F}\right)\right| e^{-\eta_{F} t\left|\operatorname{Re} \lambda_{F}\right|^{2}} \leq C A_{t} t^{-\frac{\ell^{F}}{2}}\left(\frac{|H|}{t}\right)^{\left|\Sigma^{++}\right|-\left|\Sigma_{F}^{++}\right|},
$$

where $\kappa_{F}=\frac{\left|H_{F}\right|}{2 t} \in\left[0, \kappa_{2}\right], \eta_{F}=\frac{1+\eta}{2} \in(\eta, 1), A_{t}=\sup _{|\operatorname{Im} \lambda| \leq \kappa_{2}^{\prime}}|\Omega(\lambda)| e^{-\eta t|\operatorname{Re} \lambda|^{2}}$ and $C$ is a nonnegative constant, which may depend on $\kappa_{1}, \kappa_{2}, \delta, \varepsilon, \eta$ or $p^{F}$ 
but not on $\kappa_{2}^{\prime}, \Omega, t$ or $H$ (subject to all conditions above). Let us elaborate. First of all $\Omega_{F ; t, H}\left(\lambda_{F}\right)$ is well defined when $\left|\operatorname{Im} \lambda_{F}\right| \leq \kappa_{F}$, since

$$
\begin{gathered}
\left|\operatorname{Im} \lambda_{F}+\frac{H^{F}}{2 t}\right| \leq \frac{|H|}{2 t} \leq \kappa_{2}^{\prime}, \\
\omega^{F}\left(\operatorname{Im} \lambda_{F}+\frac{H^{F}}{2 t}\right) \geq \frac{\omega^{F}\left(H^{F}\right)}{2 t}-\left|\operatorname{Im} \lambda_{F}\right| \geq \frac{\omega^{F}(H)-\left|H_{F}\right|}{2 t} \geq \frac{\left(\varepsilon-c_{1} \delta\right)|H|}{2 t} \geq 0,
\end{gathered}
$$

and hence neither $\Omega$ nor $b^{F}$ makes a problem in (3.19). Next $\Omega_{F ; t, H}$ is clearly $W_{F}$-invariant and has (at most) polynomial growth. Finally (3.20) follows from the estimates

$$
\begin{aligned}
& \left|p^{F}\left(i \lambda^{F}-\frac{H^{F}}{2 t}-\varrho^{F}\right)\right| \leq C\left(1+\left|\lambda^{F}\right|\right)^{\operatorname{deg} p^{F}}, \\
& \left|\mathbf{b}^{F}\left(-\lambda_{F}-\lambda^{F}-i \frac{H^{F}}{2 t}\right)\right|^{-1} \leq C\left(1+\left|\lambda_{F}\right|\right)^{m / 2}\left(1+\left|\lambda^{F}\right|\right)^{m / 2}, \\
& \left|\boldsymbol{\pi}^{F}\left(\frac{H^{F}}{2 t}-i \lambda_{F}-i \lambda^{F}\right)\right| \leq C\left(\frac{|H|}{t}\right)^{\left|\Sigma^{++}\right|-\left|\Sigma_{F}^{++}\right|} \\
& \quad \times\left(1+\sqrt{t}\left|\lambda_{F}\right|\right)^{\left|\Sigma^{++}\right|-\left|\Sigma_{F}^{++}\right|}\left(1+\sqrt{t}\left|\lambda^{F}\right|\right)^{\left|\Sigma^{++}\right|-\left|\Sigma_{F}^{++}\right|},
\end{aligned}
$$

the last one being established like (3.14) and the constants $C$ depending possibly on $\kappa_{1}, \kappa_{2}, \delta, \varepsilon$ or $p^{F}$ but not on $t$ or $H$. This proves our claim about $\Omega_{F ; t, H}$. By applying the induction hypothesis to the integral in (3.18), we obtain the desired estimate

$$
\left|E_{0}^{F}\right| \leq C A_{t} t^{-\frac{\ell}{2}-\mid \Sigma^{++}}\left\{\prod_{\alpha \in \Sigma^{++}}(1+\langle\alpha, H\rangle)\right\} e^{-|\varrho|^{2} t-\langle\varrho, H\rangle-\frac{|H|^{2}}{4 t}} .
$$

The other terms $E_{q}^{F}$ in (3.17) are easier to estimate. After moving the contour of integration, they become

$$
\begin{aligned}
& E_{q}^{F}=\frac{c_{2}}{\left|W_{F}\right|} e^{-|\varrho|^{2} t-\left\langle\varrho^{F}, H\right\rangle+\frac{\left|H^{F}\right|^{2}}{4 t}} \\
& \times \int_{\mathfrak{a}} d \lambda\left|\mathbf{c}_{F}\left(\lambda_{F}\right)\right|^{-2} \mathbf{c}^{F}\left(-\lambda-i \frac{H^{F}}{2 t}\right)^{-1} \times \Omega\left(\lambda+i \frac{H^{F}}{2 t}\right) e^{-t|\lambda|^{2}-i\left\langle\lambda, H^{F}\right\rangle} \\
& \quad \times p^{F}\left(i \lambda^{F}-\frac{H^{F}}{2 t}-\varrho^{F}-q^{F}\right) \varphi_{\lambda+i \frac{H^{F}}{2 t}, q}^{F}\left(\exp H: D_{F}\right) .
\end{aligned}
$$

Hence, using Theorem 2.2.8.iii and (3.16),

$$
\begin{aligned}
\left|E_{q}^{F}\right| & \leq C A_{t} e^{-|\varrho|^{2} t+\frac{\varepsilon}{4}|H|-\langle\varrho+q, H\rangle-\frac{\left|H^{F}\right|^{2}}{4 t}} \\
& \leq C A_{t} e^{-\frac{\varepsilon}{2}|H|} e^{-|\varrho|^{2} t-\langle\varrho, H\rangle-\frac{|H|^{2}}{4 t}}
\end{aligned}
$$

where the constant $C$ may depend on $\kappa_{1}, \kappa_{2}, \delta, \varepsilon, N, \eta$ or $D=D_{F} \partial\left(p^{F}\right)$ but not on $\kappa_{2}^{\prime}, \Omega, t$ or $H$. The remainder $R_{N}^{F}$ in (3.17) is also easy to estimate, using Theorem 2.2.8.iv and the assumption $N \geq 1+\frac{\kappa_{2}}{2 \varepsilon}$ :

$$
\left|R_{N}^{F}\right| \leq C \int_{\mathfrak{a}} \frac{d \lambda}{|\mathbf{c}(\lambda)|^{2}}|\Omega(\lambda)| e^{-t\left(|\varrho|^{2}+|\lambda|^{2}\right)}(1+|\lambda|)^{d}
$$




$$
\begin{aligned}
& \times(1+|H|)^{d} e^{-\langle\varrho, H\rangle-N \omega^{F}(H)} \\
\leq C & A_{t}(1+|H|)^{d} e^{-\varepsilon|H|} e^{-|\varrho|^{2} t-\langle\varrho, H\rangle-\frac{\kappa_{2}}{2}|H|} \\
\times & \int_{\mathfrak{a}} \frac{d \lambda}{|\mathbf{c}(\lambda)|^{2}}(1+|\lambda|)^{d} e^{-(1-\eta) t|\lambda|^{2}} \\
\leq C & A_{t} e^{-\frac{\varepsilon}{2}|H|} e^{-|\varrho|^{2} t-\langle\varrho, H\rangle-\frac{|H|^{2}}{4 t}} .
\end{aligned}
$$

By putting (3.17), (3.21), (3.22) and (3.23) together, we obtain eventually

$$
\left|h_{\Omega ; t}\left(\exp H: D_{F} \partial\left(p^{F}\right)\right)\right| \leq C A_{t} t^{-\frac{\ell}{2}-\mid \Sigma^{++}}\left\{\prod_{\alpha \in \Sigma^{++}}(1+\langle\alpha, H\rangle)\right\} e^{-|\varrho|^{2} t-\langle\varrho, H\rangle-\frac{|H|^{2}}{4 t}} .
$$

This estimate extends to all derivatives $h_{\Omega ; t}(\exp H: D)$ by an argument similar to the one used at the end of Step 2. For every $D \in \mathrm{U}(\mathfrak{g})$, there exist indeed differential operators $D_{1}, \ldots, D_{N} \in \mathrm{U}\left(\mathfrak{m}^{F}\right)$ of degree $\leq \operatorname{deg} D$ and continuous functions $a_{1}, \ldots, a_{N}$ on $\left\{H \in \overline{\mathfrak{a}^{+}} \mid \omega^{F}(H)>0\right\}$, which are bounded when $\omega^{F}(H) \geq \varepsilon$, such that

$$
f(\exp H: D)=\sum_{j=1}^{N} a_{j}(H) f\left(\exp H: D_{j}\right)
$$

for all $f \in C^{\infty}(G)^{\natural}$ and $H \in \overline{\mathfrak{a}^{+}}$with $\omega^{F}(H)>0$ (see for instance [GV, Lemma 7.1.1]).

This concludes the proof of the upper estimates in Theorem 3.7 and Proposition 3.9. Let us turn to the lower estimate in Theorem 3.7.

Step 5: Lower estimate for $t \gg 1+|H|^{2}$. We will obtain here the lower bound by elementary means, as in Step 1, under the assumption that $\frac{t}{1+|H|^{2}}$ is large. Let us first rescale

$$
h_{t}(\exp H)=\frac{c_{2}}{|W|} t^{-\frac{\ell}{2}-\left|\Sigma^{++}\right|} e^{-|\varrho|^{2} t} \int_{\mathfrak{a}} d \lambda \boldsymbol{\pi}(\lambda)^{2}\left|\mathbf{b}\left(\frac{\lambda}{\sqrt{t}}\right)\right|^{-2} e^{-|\lambda|^{2}} \varphi_{\frac{\lambda}{\sqrt{t}}}(\exp H)
$$

and next decompose

$$
\begin{aligned}
h_{t}(\exp H)= & I I+I I I, \\
I= & \frac{c_{2}}{|W|} \mathbf{b}(0)^{-2} t^{-\frac{\ell}{2}-\left|\Sigma^{++}\right|} e^{-|\varrho|^{2} t} \varphi_{0}(\exp H) \int_{\mathfrak{a}} d \lambda \boldsymbol{\pi}(\lambda)^{2} e^{-|\lambda|^{2}}, \\
I I= & \frac{c_{2}}{|W|} \mathbf{b}(0)^{-2} t^{-\frac{\ell}{2}-\left|\Sigma^{++}\right|} e^{-|\varrho|^{2} t} \\
\quad & \quad \times \int_{\mathfrak{a}} d \lambda \boldsymbol{\pi}(\lambda)^{2} e^{-|\lambda|^{2}}\left\{\varphi_{\frac{\lambda}{\sqrt{t}}}(\exp H)-\varphi_{0}(\exp H)\right\}, \quad \text { and } \\
I I I= & \frac{c_{2}}{|W|} t^{-\frac{\ell}{2}-\left|\Sigma^{++}\right|} e^{-|\varrho|^{2} t} \\
& \quad \times \int_{\mathfrak{a}} d \lambda \boldsymbol{\pi}(\lambda)^{2}\left\{\left|\mathbf{b}\left(\frac{\lambda}{\sqrt{t}}\right)\right|^{-2}-\mathbf{b}(0)^{-2}\right\} e^{-|\lambda|^{2}} \varphi_{\frac{\lambda}{\sqrt{t}}}(\exp H) .
\end{aligned}
$$


Notice that $I$ is a positive expression. We claim that $I I$ and $I I I$ are small in comparison, let say $|I I| \leq \frac{1}{3} I$ and $|I I I| \leq \frac{1}{3} I$, provided $\frac{t}{1+|H|^{2}}$ is sufficiently large. This follows indeed from the estimates

$$
\begin{gathered}
\varphi_{\frac{\lambda}{\sqrt{t}}}(\exp H)-\varphi_{0}(\exp H)=\mathrm{O}\left(t^{-1 / 2}|\lambda||H| \varphi_{0}(\exp H)\right), \\
\left|\mathbf{b}\left(\frac{\lambda}{\sqrt{t}}\right)\right|^{-2}-\mathbf{b}(0)^{-2}=\mathrm{O}\left(t^{-1 / 2}|\lambda|(1+|\lambda|)^{m}\right)
\end{gathered}
$$

which are easily deduced from (2.2.1), respectively (2.2.5) and (2.2.6). As a conclusion we obtain

$$
h_{t}(\exp H) \geq \frac{1}{3} I \geq C t^{-\frac{\ell}{2}-\left|\Sigma^{++}\right|} e^{-|\varrho|^{2} t} \varphi_{o}(\exp H),
$$

which is comparable to the expected bound

$$
t^{-\frac{\ell}{2}-\mid \Sigma^{++}}\left\{\prod_{\alpha \in \Sigma^{++}}(1+\langle\alpha, H\rangle)\right\} e^{-|\varrho|^{2} t-\langle\alpha, H\rangle-\frac{|H|^{2}}{4 t}}
$$

according to Proposition 2.2.12.i and to the assumption $|H|=\mathrm{O}(\sqrt{t})$.

Step 6: Lower estimate away from the walls. We shall restrict here to $t>0$ and $H \in \mathfrak{a}^{+}$with $\omega(H)$ large. Let us resume the computations carried out in Step 2. We have

where

$$
h_{t}(\exp H)=\sum_{q \in 2 Q} E_{q}
$$

$$
E_{0}=c_{2} e^{-|\varrho|^{2} t-\langle\varrho, H\rangle} \int_{\mathfrak{a}} d \lambda e^{-t|\lambda|^{2}} \boldsymbol{\pi}\left(-\frac{i}{2 t} \frac{\partial}{\partial \lambda}\right)\left\{\mathbf{b}(-\lambda)^{-1} e^{i\langle\lambda, H\rangle}\right\}
$$

and

$$
\left|E_{q}\right| \leq C(1+|q|)^{d} t^{-\frac{\ell}{2}} \mathbf{c}\left(-i \frac{H}{t}\right)^{-1} e^{-|\varrho|^{2} t-\langle\varrho+q, H\rangle-\frac{|H|^{2}}{4 t}},
$$

the constant $C$ depending possibly on a lower bound $\kappa>0$ for $\omega(H)$ but not on $q, t$ or $H$ (subject to the conditions above). Moreover

$$
\begin{gathered}
E_{0}=c_{2} t^{-\frac{\ell}{2}} e^{-|\varrho|^{2} t-\langle\varrho, H\rangle-\frac{|H|^{2}}{4 t}} \\
\times \sum_{\Sigma^{++}=\Sigma^{\prime} \sqcup \Sigma^{\prime \prime}}\left\{\prod_{\alpha^{\prime} \in \Sigma^{\prime}} \frac{\left\langle\alpha^{\prime}, H\right\rangle}{2 t}\right\} \int_{\mathfrak{a}} d \lambda e^{-|\lambda|^{2}}\left\{\prod_{\alpha^{\prime \prime} \in \Sigma^{\prime \prime}}\left(-\frac{i}{2 t} \partial_{\alpha^{\prime \prime}}\right)\right\}\left(\mathbf{b}^{-1}\right)\left(-\frac{\lambda}{\sqrt{t}}-i \frac{H}{2 t}\right) \\
=I_{0}+I I_{0}+I I I_{0},
\end{gathered}
$$

where

$$
\begin{aligned}
I_{0}=c_{2} & \underbrace{\left\{\int_{\mathfrak{a}} d \lambda e^{-|\lambda|^{2}}\right\}}_{\pi^{\ell / 2}} t^{-\frac{\ell}{2}} \mathbf{c}\left(-i \frac{H}{2 t}\right)^{-1} e^{-|\varrho|^{2} t-\langle\varrho, H\rangle-\frac{|H|^{2}}{4 t}}, \\
I I_{0}=c_{2} t^{-\frac{\ell}{2}} \boldsymbol{\pi}\left(\frac{H}{2 t}\right) e^{-|\varrho|^{2} t-\langle\varrho, H\rangle-\frac{|H|^{2}}{4 t}} & \times \int_{\mathfrak{a}} d \lambda e^{-|\lambda|^{2}}\left\{\mathbf{b}\left(-\frac{\lambda}{\sqrt{t}}-i \frac{H}{2 t}\right)^{-1}-\mathbf{b}\left(-i \frac{H}{2 t}\right)^{-1}\right\}
\end{aligned}
$$


and the remaining sum over $\Sigma^{\prime} \subsetneq \Sigma^{++}$verifies

$$
\left|I I I_{0}\right| \leq C \omega(H)^{-1} t^{-\frac{\ell}{2}} \mathbf{c}\left(-i \frac{H}{t}\right)^{-1} e^{-|\varrho|^{2} t-\langle\varrho, H\rangle-\frac{|H|^{2}}{4 t}},
$$

with a constant $C$ as above. Let us establish a similar estimate for $I I_{0}$ with $\omega(H)^{-\frac{1}{2}}$ instead of $\omega(H)^{-1}$. Since

$$
\left|\mathbf{b}\left(-\frac{\lambda}{\sqrt{t}}-i \frac{H}{2 t}\right)^{-1}-\mathbf{b}\left(-i \frac{H}{2 t}\right)^{-1}\right| \leq \frac{|\lambda|}{\sqrt{t}} \sup _{\lambda^{\prime} \in\left[0, \frac{\lambda}{\sqrt{t}}\right]}\left|\nabla\left(\mathbf{b}^{-1}\right)\left(-\lambda^{\prime}-i \frac{H}{2 t}\right)\right|,
$$

we are left with a gradient estimate of $\mathbf{b}^{-1}$. When $\omega(H) \leq t$, we use simply (3.11) and obtain

$$
\mathbf{b}\left(-\frac{\lambda}{\sqrt{t}}-i \frac{H}{2 t}\right)^{-1}-\mathbf{b}\left(-i \frac{H}{2 t}\right)^{-1}=\mathrm{O}\left(\omega(H)^{-\frac{1}{2}} \mathbf{b}\left(-i \frac{H}{t}\right)^{-1}(1+|\lambda|)^{\frac{m}{2}+1}\right) .
$$

Recall that (3.11) relied on (2.2.6), which was itself deduced from (2.2.5) by applying Cauchy's formula to $\mathbf{b}^{-1}$ in a small torus. When $\omega(H) \geq t$, we can use a larger torus $\mathbb{T}^{\ell} \subset \mathfrak{a}-i \overline{\mathfrak{a}^{+}}$, centered at the point $-\lambda^{\prime}-i \frac{H}{2 t}$ and of radius $r$ proportional to $\omega(H) / t$ (for instance $r=\omega(H) / 2 \sqrt{\ell} t$ ) and obtain this way

$$
\nabla\left(\mathbf{b}^{-1}\right)\left(-\lambda^{\prime}-i \frac{H}{2 t}\right)=\mathrm{O}\left(\frac{t}{\omega(H)} \mathbf{b}\left(-i \frac{H}{t}\right)^{-1}\left(1+\sqrt{t}\left|\lambda^{\prime}\right|\right)^{m / 2}\right)
$$

hence

$$
\mathbf{b}\left(-\frac{\lambda}{\sqrt{t}}-i \frac{H}{2 t}\right)^{-1}-\mathbf{b}\left(-i \frac{H}{2 t}\right)^{-1}=\mathrm{O}\left(\omega(H)^{-\frac{1}{2}} \mathbf{b}\left(-i \frac{H}{t}\right)^{-1}(1+|\lambda|)^{\frac{m}{2}+1}\right) .
$$

Thus in both cases

$$
\left|I I_{0}\right| \leq C \omega(H)^{-\frac{1}{2}} t^{-\frac{\ell}{2}} \mathbf{c}\left(-i \frac{H}{t}\right)^{-1} e^{-|\varrho|^{2} t-\langle\varrho, H\rangle-\frac{|H|^{2}}{4 t}},
$$

with a constant $C$ as above. As a conclusion, $I_{0}$ proves to be the leading term in the expansion

$$
h_{t}(\exp H)=I_{0}+I I_{0}+I I I_{0}+\sum_{q \in 2 Q \backslash\{0\}} E_{q},
$$

as $\omega(H) \rightarrow+\infty$, and in particular

$$
h_{t}(\exp H) \geq \frac{1}{2} I_{0} \geq C t^{-\frac{\ell}{2}} \mathbf{c}\left(-i \frac{H}{t}\right)^{-1} e^{-|\varrho|^{2} t-\langle\varrho, H\rangle-\frac{|H|^{2}}{4 t}}
$$

provided $\omega(H)$ is sufficiently large. This concludes Step 6 .

Step \%: Lower estimate along faces $I$. We shall restrict here to $t>0$ and $H \in \overline{\mathfrak{a}^{+}}(F, \delta, \varepsilon)$ with $\kappa_{1} \leq|H| \leq \kappa_{2} \sqrt{t}$, where $\kappa_{1} \in(0,+\infty)$ is large, $\kappa_{2} \in(0,+\infty)$ is arbitrary, $0<\delta \leq \varepsilon<+\infty$ are arbitrarily small, as well as $\delta / \varepsilon$, and $F$ denotes any nontrivial subset of $\Sigma^{+++}$. We will expand again the heat kernel along faces, but differently from Step 4. This time we need only the first order expansion in Theorem 2.2.8, we use no induction argument and we perform an integration by parts as in Step 2. To begin with, as in Step 4 we deduce easily from Theorem 2.2.8 that

$$
h_{t}(\exp H)=E^{F}+R^{F} \text {, }
$$


where the leading term writes

$$
E^{F}=c_{2}\left|W_{F}\right|^{-1} e^{-|\varrho|^{2} t-\left\langle\varrho^{F}, H\right\rangle} \int_{\mathfrak{a}} d \lambda\left|\mathbf{c}_{F}(\lambda)\right|^{-2} \mathbf{c}^{F}(-\lambda)^{-1} e^{-t|\lambda|^{2}} \varphi_{\lambda}^{F}(\exp H)
$$

and the remainder can be estimated as follows:

$$
\left|R^{F}\right| \leq C e^{-\frac{\varepsilon}{2}|H|} e^{-|\varrho|^{2} t-\langle\varrho, H\rangle},
$$

with a constant $C \geq 0$ depending possibly on $\varepsilon$ and on lower bounds for $t$ and $|H|$, hence on $\kappa_{2}$ and on a lower bound for $\kappa_{1}$, but not on $\delta$. Let us analyze the expression $E^{F}$. By writing

$$
\left|\mathbf{c}_{F}(\lambda)\right|^{-2} \mathbf{c}^{F}(-\lambda)^{-1}=\boldsymbol{\pi}(-i \lambda) \boldsymbol{\pi}_{F}(i \lambda)\left|\mathbf{b}_{F}(\lambda)\right|^{-2} \mathbf{b}^{F}(-\lambda)^{-1}
$$

and by performing an integration by parts based on

as in Step 2, we obtain first

$$
\boldsymbol{\pi}(-i \lambda) e^{-t|\lambda|^{2}}=\boldsymbol{\pi}\left(\frac{i}{2 t} \frac{\partial}{\partial \lambda}\right) e^{-t|\lambda|^{2}}
$$

$$
\begin{aligned}
E^{F} & =c_{2} 2^{-\left|\Sigma^{++}\right|}\left|W_{F}\right|^{-1} t^{-\left|\Sigma^{++}\right|} e^{-|\varrho|^{2} t-\left\langle\varrho^{F}, H\right\rangle} \\
& \times \int_{\mathfrak{a}} d \lambda e^{-t|\lambda|^{2}} \boldsymbol{\pi}\left(-i \frac{\partial}{\partial \lambda}\right)\left\{\boldsymbol{\pi}_{F}(i \lambda)\left|\mathbf{b}_{F}(\lambda)\right|^{-2} \mathbf{b}^{F}(-\lambda)^{-1} \varphi_{\lambda}^{F}(\exp H)\right\} .
\end{aligned}
$$

By using

by expanding

$$
\varphi_{\lambda}^{F}(\exp H)=\varphi_{\lambda}^{F}\left(\exp H_{F}\right) e^{i\left\langle\lambda, H^{F}\right\rangle}
$$

$$
\begin{aligned}
& \boldsymbol{\pi}\left(-i \frac{\partial}{\partial \lambda}\right)\left\{\boldsymbol{\pi}_{F}(i \lambda)\left|\mathbf{b}_{F}(\lambda)\right|^{-2} \mathbf{b}^{F}(-\lambda)^{-1} \varphi_{\lambda}^{F}(\exp H)\right\} \\
& =\sum_{\Sigma^{++} \backslash \Sigma_{F}^{++}=\Sigma^{\prime} \sqcup \Sigma^{\prime \prime}}\left\{\prod_{\alpha^{\prime} \in \Sigma^{\prime}}\left\langle\alpha^{\prime}, H^{F}\right\rangle\right\} e^{i\left\langle\lambda, H^{F}\right\rangle} \\
& \times\left\{\prod_{\alpha^{\prime \prime} \in \Sigma^{\prime \prime} \sqcup \Sigma_{F}^{++}}\left(-i \partial_{\alpha^{\prime \prime}}\right)\right\}\left\{\boldsymbol{\pi}_{F}(i \lambda)\left|\mathbf{b}_{F}(\lambda)\right|^{-2} \mathbf{b}^{F}(-\lambda)^{-1} \varphi_{\lambda}^{F}\left(\exp H_{F}\right)\right\}
\end{aligned}
$$

and by moving the contour of integration, we obtain next

$$
\begin{aligned}
& E^{F}=c_{2} 2^{-\left|\Sigma^{++}\right|}\left|W_{F}\right|^{-1} t^{-\left|\Sigma^{++}\right|} e^{-|\varrho|^{2} t-\left\langle\varrho^{F}, H\right\rangle-\frac{\left|H^{F}\right|^{2}}{4 t}} \\
& \times \sum_{\Sigma^{++} \backslash \Sigma_{F}^{++}=\Sigma^{\prime} \sqcup \Sigma^{\prime \prime}}\left\{\prod_{\alpha^{\prime} \in \Sigma^{\prime}}\left\langle\alpha^{\prime}, H^{F}\right\rangle\right\} \times \int_{\mathfrak{a}} d \lambda e^{-t|\lambda|^{2}}\left\{\prod_{\alpha^{\prime \prime} \in \Sigma^{\prime \prime} \sqcup \Sigma_{F}^{++}}\left(-i \partial_{\alpha^{\prime \prime}}\right)\right\} \\
& \quad \times\left\{\boldsymbol{\pi}_{F}(i \lambda)\left|\mathbf{b}_{F}(\lambda)\right|^{-2} \mathbf{b}^{F}\left(-\lambda-i \frac{H^{F}}{2 t}\right)^{-1} \varphi_{\lambda}^{F}\left(\exp H_{F}\right)\right\} . \quad(3.26)
\end{aligned}
$$

Let us concentrate on the expression

$$
\begin{aligned}
c_{4}^{F} t^{-\left|\Sigma^{++}\right|} & \boldsymbol{\pi}^{F}\left(H^{F}\right) e^{-|\varrho|^{2} t-\left\langle\varrho^{F}, H\right\rangle-\frac{\left|H^{F}\right|^{2}}{4 t}} \\
& \times \int_{\mathfrak{a}} d \lambda e^{-t|\lambda|^{2}}\left|\mathbf{b}_{F}(\lambda)\right|^{-2} \mathbf{b}^{F}\left(-\lambda-i \frac{H^{F}}{2 t}\right)^{-1} \varphi_{\lambda}^{F}\left(\exp H_{F}\right),
\end{aligned}
$$

with

$$
c_{4}^{F}=c_{2} 2^{-\left|\Sigma^{++}\right|}\left|W_{F}\right|^{-1} \partial\left(\boldsymbol{\pi}_{F}\right) \boldsymbol{\pi}_{F}=c_{2} 2^{-\left|\Sigma^{++}\right|} \boldsymbol{\pi}_{F}(\widetilde{\varrho}),
$$


which will prove to be the main term in (3.26), and which is obtained by taking $\Sigma^{\prime}=\Sigma^{++} \backslash \Sigma_{F}^{++}$, i.e. $\Sigma^{\prime \prime}=\emptyset$ in the above sum and by applying all remaining derivatives $\boldsymbol{\pi}_{F}\left(-i \frac{\partial}{\partial \lambda}\right)=\prod_{\alpha^{\prime \prime} \in \Sigma_{F}^{++}}\left(-i \partial_{\alpha^{\prime \prime}}\right)$ to the factor $\boldsymbol{\pi}_{F}(i \lambda)$. We decompose the integral in (3.27) as a sum

$$
I^{F}+I I^{F}+I I I^{F}
$$

where

$$
\begin{aligned}
& I^{F}=\mathbf{b}_{F}(0)^{-2} \mathbf{b}^{F}(0)^{-1} \varphi_{0}^{F}\left(\exp H_{F}\right) \underbrace{\int_{\mathfrak{a}} d \lambda e^{-t|\lambda|^{2}}}_{(\pi / t)^{\ell / 2}}, \\
& I I^{F}=\mathbf{b}_{F}(0)^{-2} \mathbf{b}^{F}(0)^{-1} \int_{\mathfrak{a}} d \lambda e^{-t|\lambda|^{2}}\left\{\varphi_{\lambda}^{F}\left(\exp H_{F}\right)-\varphi_{0}^{F}\left(\exp H_{F}\right)\right\}, \\
& I I I^{F}=\int_{\mathfrak{a}} d \lambda e^{-t|\lambda|^{2}}\left\{\left|\mathbf{b}_{F}(\lambda)\right|^{-2} \mathbf{b}^{F}\left(-\lambda-i \frac{H^{F}}{2 t}\right)^{-1}\right. \\
&\left.-\left|\mathbf{b}_{F}(0)\right|^{-2} \mathbf{b}^{F}(0)^{-1}\right\} \varphi_{\lambda}^{F}\left(\exp H_{F}\right) .
\end{aligned}
$$

Using the estimates

$$
\begin{gathered}
\left|\varphi_{\lambda}^{F}\left(\exp H_{F}\right)-\varphi_{0}^{F}\left(\exp H_{F}\right)\right| \leq|\lambda|\left|H_{F}\right| \varphi_{0}^{F}\left(\exp H_{F}\right) \leq c_{1} \delta|\lambda||H| \varphi_{0}^{F}(\exp H), \\
\left|\mathbf{b}_{F}(\lambda)\right|^{-2} \mathbf{b}^{F}\left(-\lambda-i \frac{H^{F}}{2 t}\right)^{-1} a-\left|\mathbf{b}_{F}(0)\right|^{-2} \mathbf{b}^{F}(0)^{-1}=\mathrm{O}\left(\left\{|\lambda|+\frac{|H|}{t}\right\}(1+|\lambda|)^{m}\right),
\end{gathered}
$$

which follow from (2.2.1) and Lemma 2.1.7.i , respectively (2.2.5) and (2.2.6), we get

$$
\begin{aligned}
\left|I I^{F}\right| & \leq C \delta t^{-\ell / 2} \varphi_{0}^{F}(\exp H), \\
\left|I I I^{F}\right| & \leq C t^{-\frac{\ell+1}{2}} \varphi_{0}^{F}(\exp H),
\end{aligned}
$$

with constants $C \geq 0$ depending on $\kappa_{2}$ and on a lower bound for $t$, hence for $\kappa_{1}$, but not on $\delta$ or $\varepsilon$. Thus $I^{F}$ is the main term in (3.27) and we have for instance

$$
\left|I I^{F}\right|+\left|I I I^{F}\right| \leq \frac{1}{2} I^{F}
$$

provided $\delta$ is sufficiently small and $\kappa_{1}$ hence $t$ is sufficiently large. As a consequence (3.27) is bounded below by

$$
\begin{aligned}
& c_{4}^{F} t^{-\left|\Sigma^{++}\right|} \pi^{F}\left(H^{F}\right) e^{-|\varrho|^{2} t-\left\langle\varrho^{F}, H\right\rangle-\frac{\left|H^{F}\right|^{2}}{4 t}} \times \frac{1}{2} I^{F} \\
& \geq C t^{-\frac{\ell}{2}-\left|\Sigma^{++}\right|} \boldsymbol{\pi}^{F}\left(H^{F}\right) \varphi_{0}^{F}(\exp H) e^{-|\varrho|^{2} t-\left\langle\varrho^{F}, H\right\rangle},
\end{aligned}
$$

with a constant $C>0$ depending on $\kappa_{2}$, but not on $\kappa_{1}, \delta$ or $\varepsilon$. We claim that the other terms in (3.26) are

$$
\mathrm{O}\left(\left\{\delta+\frac{\delta}{\varepsilon}+\frac{1}{\varepsilon \kappa_{1}}+t^{-\frac{1}{2}}\right\} t^{-\frac{\ell}{2}-\left|\Sigma^{++}\right|} \boldsymbol{\pi}^{F}\left(H^{F}\right) \varphi_{0}^{F}(\exp H) e^{-|\varrho|^{2} t-\left\langle\varrho^{F}, H\right\rangle}\right),
$$

with the constant in $\mathrm{O}$ depending possibly on $\kappa_{2}$, on a lower bound for $\kappa_{1}$ and on an upper bound for $\varepsilon$, but not on $\delta$. The proof relies on the 
estimates

$$
\begin{aligned}
& \left(\frac{\partial}{\partial \lambda}\right)^{\sigma} \boldsymbol{\pi}_{F}(i \lambda)=\left\{\begin{array}{cll}
\mathrm{O}\left(|\lambda|^{\left|\Sigma_{F}^{++}\right|-|\sigma|}\right) & \text { if } & |\sigma| \leq\left|\Sigma_{F}^{++}\right|, \\
0 & \text { if } & |\sigma|>\left|\Sigma_{F}^{++}\right|,
\end{array}\right. \\
& \left(\frac{\partial}{\partial \lambda}\right)^{\sigma}\left\{\left|\mathbf{b}_{F}(\lambda)\right|^{-2} \mathbf{b}^{F}\left(-\lambda-i \frac{H^{F}}{2 t}\right)^{-1}\right\}=\mathrm{O}\left(\{1+|\lambda|\}^{m}\right), \\
& \left(\frac{\partial}{\partial \lambda}\right)^{\sigma} \varphi_{\lambda}^{F}\left(\exp H_{F}\right)=\mathrm{O}\left(\left|H_{F}\right|^{|\sigma|} \varphi_{0}^{F}(\exp H)\right) .
\end{aligned}
$$

(3.31.1) is immediate, (3.31.2) follows again from (2.2.5) and (2.2.6), and (3.31.3) from (2.2.1). Let us elaborate on (3.30). Consider first the terms in (3.26) which are obtained by taking $\Sigma^{\prime}=\Sigma^{++} \backslash \Sigma_{F}^{++}$and by applying some derivatives $\partial_{\alpha^{\prime \prime}}$ to $\left|\mathbf{b}_{F}(\lambda)\right|^{-2} \mathbf{b}^{F}\left(-\lambda-i \frac{H^{F}}{2 t}\right)^{-1} \varphi_{\lambda}^{F}\left(\exp H_{F}\right)$. Using the estimates (3.31), we get in this case an upper bound

$$
\mathrm{O}\left(\left\{\frac{1+\left|H_{F}\right|}{\sqrt{t}}\right\}^{N} t^{-\frac{\ell}{2}-\left|\Sigma^{++}\right|} \boldsymbol{\pi}^{F}\left(H^{F}\right) \varphi_{0}^{F}(\exp H) e^{-|\varrho|^{2} t-\left\langle\varrho^{F}, H\right\rangle}\right)
$$

as expected, with $\left\{\frac{1+\left|H_{F}\right|}{\sqrt{t}}\right\}^{N}=\mathrm{O}\left(t^{-\frac{1}{2}}+\delta\right)$ since $\left|H_{F}\right| \leq c_{1} \delta|H|$ (Lemma 2.1.7.i) and $\kappa_{1} \leq|H| \leq \kappa_{2} \sqrt{t}$ by assumption. Consider next the nonzero terms in (3.26) with $\Sigma^{\prime} \subsetneq \Sigma^{++} \backslash \Sigma_{F}^{++}$. In this case there are on one hand some missing factors $\left\langle\alpha^{\prime}, H^{F}\right\rangle$ in $\boldsymbol{\pi}^{F}\left(H^{F}\right)$, and on the other hand at least as many derivatives $\partial_{\alpha^{\prime \prime}}$ applied to $\left|\mathbf{b}_{F}(\lambda)\right|^{-2} \mathbf{b}^{F}\left(-\lambda-i \frac{H^{F}}{2 t}\right)^{-1} \varphi_{\lambda}^{F}\left(\exp H_{F}\right)$. Using the estimates (3.31), we get this time an upper bound

$$
\mathrm{O}\left(\left\{\frac{1+\left|H_{F}\right|}{\left\langle\alpha^{\prime}, H^{F}\right\rangle}\right\}^{N} t^{-\frac{\ell}{2}-\left|\Sigma^{++}\right|} \pi^{F}\left(H^{F}\right) \varphi_{0}^{F}(\exp H) e^{-|\varrho|^{2} t-\left\langle\varrho^{F}, H\right\rangle}\right)
$$

with $\left\{\frac{1+\left|H_{F}\right|}{\left\langle\alpha^{\prime}, H^{F}\right\rangle}\right\}^{N}=\mathrm{O}\left(\frac{1}{\varepsilon \kappa_{1}}+\frac{\delta}{\varepsilon}\right)$, since $\left\langle\alpha^{\prime}, H^{F}\right\rangle \geq \omega^{F}\left(H^{F}\right) \geq \omega^{F}(H) \geq$ $\varepsilon|H| \geq \varepsilon \kappa_{1}$ and $\left|H_{F}\right| \leq c_{1} \delta|H|$. This proves our claim (3.30). By putting everything together, we can now conclude that

$$
h_{t}(\exp H) \geq C t^{-\frac{\ell}{2}-\left|\Sigma^{++}\right|} \pi^{F}\left(H^{F}\right) \varphi_{0}^{F}(\exp H) e^{-|\varrho|^{2} t-\left\langle\varrho^{F}, H\right\rangle} .
$$

More precisely, given $\varepsilon$ and $\kappa_{2}$, we choose $\delta$ sufficiently small and $\kappa_{1}$ sufficiently large so that (3.29) holds, which implies the lower estimate (3.32) for the expression (3.27). By possibly reducing $\delta$ or increasing $\kappa_{1}$, this estimate can be extended to $E^{F}$ and furthermore to $h_{t}(\exp H)$, according to (3.30) and (3.25). Finally notice that, under the present assumptions, the right hand side of (3.32) is comparable to the expected bound

$$
t^{-\frac{\ell}{2}-\mid \Sigma^{++}}\left\{\prod_{\alpha \in \Sigma^{++}}(1+\langle\alpha, H\rangle)\right\} e^{-|\varrho|^{2} t-\langle\alpha, H\rangle-\frac{|H|^{2}}{4 t}} .
$$

This follows from Proposition 2.2.12.i, applied to $\varphi_{0}^{F}$, and from the fact that the expressions

$$
\prod_{\alpha \in \Sigma^{++} \backslash \Sigma_{F}^{++}}(1+\langle\alpha, .\rangle), \boldsymbol{\pi}^{F},\left(\omega^{F}\right)^{\left|\Sigma^{++}\right|-\left|\Sigma_{F}^{++}\right|} \text {and }|\cdot|^{\left|\Sigma^{++}\right|-\left|\Sigma_{F}^{++}\right|},
$$


applied to $H$ or $H^{F}$, are all comparable.

REMARK 3.34. Let us indicate another proof of the lower estimate along faces

$$
h_{t}(\exp H) \geq C t^{-\frac{\ell}{2}-\left|\Sigma^{++}\right|} e^{-|\varrho|^{2} t} \varphi_{0}(\exp H)
$$

when $t \geq \kappa\left(1+|H|^{2}\right)$, which is simpler than Step 7, but which has the disadvantage of yielding only estimates and no asymptotics (and which is moreover not in the spirit of asymptotic methods used throughout this paper). The idea consists in reducing to the estimate (3.35) away from the walls, which has been established in Step 6. Take $H_{0} \in \mathfrak{a}^{+}$at distance $>2$ from the walls and consider the balls

$$
B_{r}=\left\{H \in \mathfrak{a}|| H-r H_{0} \mid \leq r\right\} \subset B_{r}^{\prime}=\left\{H \in \mathfrak{a}|| H-r H_{0} \mid \leq 2 r\right\}
$$

in $\mathfrak{a}^{+}$. According to Lemma 2.1.2, the set $K\left(\exp B_{r}\right) K\left[\operatorname{resp} . K\left(\exp B_{r}^{\prime}\right) K\right]$ consists of all points in $\mathbb{X}=G / K$ at distance $\leq r$ [resp. $\leq 2 r]$ from the $K$-orbit $K\left(\exp r H_{0}\right) K$. Denote by $\chi_{r}\left[\right.$ resp. $\left.\chi_{r}^{\prime}\right]$ its characteristic function. Letting $r(t)=\sqrt{t / \kappa}$, we can estimate

$$
\begin{aligned}
h_{t}=h_{t / 2} * h_{t / 2} & \geq\left\{\chi_{r(t)} h_{t / 2}\right\} *\left\{\chi_{r(t)}^{\prime} h_{t / 2}\right\} \\
& \geq C t^{-\ell-2\left|\Sigma^{++}\right|} e^{-|\varrho|^{2} t}\left\{\chi_{r(t)} \varphi_{0}\right\} *\left\{\chi_{r(t)}^{\prime} \varphi_{0}\right\},
\end{aligned}
$$

using the semigroup property of the heat kernel, its positivity, and (3.35) away from the walls. On one hand

$$
\left\{\chi_{r(t)} \varphi_{0}\right\} *\left\{\chi_{r(t)}^{\prime} \varphi_{0}\right\}(\exp H)=\int_{K\left(\exp B_{r(t)}\right) K} d x \varphi_{0}\left(x^{ \pm 1}\right)\left\{\chi_{r(t)}^{\prime} \varphi_{0}\right\}(x \exp H)
$$

coincides with

$$
\left\{\chi_{r(t)} \varphi_{0}\right\} * \varphi_{0}(\exp H)=\int_{K\left(\exp B_{r(t)}\right) K} d x \varphi_{0}\left(x^{ \pm 1}\right) \varphi_{0}(x \exp H)
$$

for $|H| \leq r(t)$, since

$$
\begin{aligned}
& d\left(x(\exp H) K, K\left\{\exp r(t) H_{0}\right\} K\right) \\
& \quad \leq d(x(\exp H) K, x K)+d\left(x K, K\left\{\exp r(t) H_{0}\right\} K\right) \\
& \quad \leq|H|+r(t) \leq 2 r(t) .
\end{aligned}
$$

On the other hand

$$
\left\{\chi_{r(t)} \varphi_{0}\right\} * \varphi_{0}=\left\{\int_{K\left(\exp B_{r(t)}\right) K} d x \varphi_{0}(x)^{2}\right\} \varphi_{0}
$$

by the functional equation of $\varphi_{0}$. Thus we are left with the last brace, which is easily estimated using (2.1.5) and Proposition 2.2.12.i:

$$
\int_{K\left(\exp B_{r(t)}\right) K} d x \varphi_{0}(x)^{2} \asymp \int_{B_{r(t)}} d H^{\prime}\left|H^{\prime}\right|^{2\left|\Sigma^{++}\right|} \asymp t^{\frac{\ell}{2}+\left|\Sigma^{++}\right|} .
$$

This concludes Remark 3.34 . 
Step 8: Lower estimate along faces II. In this last step, we restrict to $t>0$ and $H \in \overline{\mathfrak{a}^{+}}(F, \delta, \varepsilon)$ with $\kappa_{1} \sqrt{t} \leq|H| \leq \kappa_{2} t$. Here $\kappa_{1} \in(0,+\infty)$ is large and $\kappa_{2} \in(0,+\infty)$ is arbitrary. As usual, $F$ denotes any nontrivial subset of $\Sigma^{+++}$and the constants $0<\delta \leq \varepsilon<+\infty$ can be taken as small as we wish, as well as $\delta / \varepsilon$. We will establish the lower estimate

$$
h_{t}(\exp H) \geq C t^{-\frac{\ell}{2}-\mid \Sigma^{++}} \mid\left\{\prod_{\alpha \in \Sigma^{++}}(1+\langle\alpha, H\rangle)\right\} e^{-|\varrho|^{2} t-\langle\alpha, H\rangle-\frac{|H|^{2}}{4 t}}
$$

by proceeding as in Step 4. Starting with the induction hypothesis, assume that the lower estimate in Theorem 3.7.ii holds for all proper symmetric subspaces with no Euclidean factor in $\mathbb{X}=G / K$. Recall the heat kernel expansion investigated in Step 4:

$$
h_{t}(\exp H)=\sum_{\substack{q \in 2 Q^{F} \\ \varkappa(q)<N}} E_{q}^{F}+R_{N}^{F}
$$

where

$$
\begin{aligned}
E_{0}^{F}=\frac{c_{2}}{\left|W_{F}\right|} e^{-|\varrho|^{2} t} & -\left\langle\varrho^{F}, H\right\rangle-\frac{\left|H^{F}\right|^{2}}{4 t} \\
& \times \int_{\mathfrak{a}_{F}} d \lambda_{F}\left|\mathbf{c}_{F}\left(\lambda_{F}\right)\right|^{-2} \Omega_{F ; t, H}\left(\lambda_{F}\right) e^{-t\left|\lambda_{F}\right|^{2}} \varphi_{\lambda_{F}}^{F}\left(\exp H_{F}\right),
\end{aligned}
$$

with

and

$$
\Omega_{F ; t, H}\left(\lambda_{F}\right)=\int_{\mathfrak{a}^{F}} d \lambda^{F} \mathbf{c}^{F}\left(-\lambda_{F}-\lambda^{F}-i \frac{H^{F}}{2 t}\right)^{-1} e^{-t\left|\lambda^{F}\right|^{2}}
$$

$$
\sum_{\substack{q \in 2 Q^{F} \\ 0<\varkappa(q)<N}}\left|E_{q}^{F}\right|+\left|R_{N}^{F}\right| \leq C e^{-\frac{\varepsilon}{2}|H|} e^{-|\varrho|^{2} t-\langle\varrho, H\rangle-\frac{|H|^{2}}{4 t}},
$$

provided $\frac{\varepsilon}{\delta} \geq\left(1+2 \kappa_{2}\right) c_{1}$ and $N \geq 1+\frac{\kappa_{2}}{4 \varepsilon}$, the constant $C$ depending possibly on $\kappa_{2}, \delta, \varepsilon, N$ and on a lower bound for $\kappa_{1}$. Thus (3.36) will follow from a similar estimate for $E_{0}^{F}$, provided $\kappa_{1}$ hence $t$ and $H$ are sufficiently large. Let us therefore concentrate on $E_{0}^{F}$. Consider the decomposition

$$
E_{0}^{F}=I_{0}^{F}+I I_{0}^{F}+I I I_{0}^{F},
$$

where

$$
\begin{aligned}
& I_{0}^{F}=\frac{c_{2}}{\left|W_{F}\right|} \underbrace{\left\{\int_{\mathfrak{a}^{F}} d \lambda^{F} e^{\left.-\left|\lambda^{F}\right|^{2}\right\}}\right.}_{\pi^{\ell F / 2}} \\
& \times t^{-\frac{\ell^{F}}{2}} \mathbf{c}^{F}\left(-i \frac{H^{F}}{2 t}\right)^{-1} e^{-|\varrho|^{2} t-\left\langle\varrho^{F}, H\right\rangle-\frac{\left|H^{F}\right|^{2}}{4 t}} \\
& \quad \times \int_{\mathfrak{a}_{F}} d \lambda_{F}\left|\mathbf{c}_{F}\left(\lambda_{F}\right)\right|^{-2} e^{-t\left|\lambda_{F}\right|^{2}} \varphi_{\lambda_{F}}^{F}\left(\exp H_{F}\right),
\end{aligned}
$$




$$
\begin{aligned}
I I_{0}^{F}= & \frac{c_{2}}{\left|W_{F}\right|} \boldsymbol{\pi}^{F}\left(\frac{H^{F}}{2 t}\right) e^{-|\varrho|^{2} t-\left\langle\varrho^{F}, H\right\rangle-\frac{\left|H^{F}\right|^{2}}{4 t}} \\
& \times \int_{\mathfrak{a}_{F}} d \lambda_{F}\left|\mathbf{c}_{F}\left(\lambda_{F}\right)\right|^{-2} \Omega_{F ; t, H}^{\prime \prime}\left(\lambda_{F}\right) e^{-t\left|\lambda_{F}\right|^{2}} \varphi_{\lambda_{F}}^{F}\left(\exp H_{F}\right), \\
I I I_{0}^{F}= & \frac{c_{2}}{\mid W_{F}} e^{-|\varrho|^{2} t-\left\langle\varrho^{F}, H\right\rangle-\frac{\left|H^{F}\right|^{2}}{4 t}} \\
& \times \int_{\mathfrak{a}_{F}} d \lambda_{F}\left|\mathbf{c}_{F}\left(\lambda_{F}\right)\right|^{-2} \Omega_{F ; t, H}^{\prime \prime \prime}\left(\lambda_{F}\right) e^{-t\left|\lambda_{F}\right|^{2}} \varphi_{\lambda_{F}}^{F}\left(\exp H_{F}\right),
\end{aligned}
$$

with

$$
\begin{gathered}
\Omega_{F ; t, H}^{\prime \prime}\left(\lambda_{F}\right)=\int_{\mathfrak{a}^{F}} d \lambda^{F}\left\{\mathbf{b}^{F}\left(-\lambda_{F}-\lambda^{F}-i \frac{H^{F}}{2 t}\right)^{-1}-\mathbf{b}^{F}\left(-i \frac{H^{F}}{2 t}\right)^{-1}\right\} e^{-t\left|\lambda^{F}\right|^{2}}, \\
\Omega_{F ; t, H}^{\prime \prime \prime}\left(\lambda_{F}\right)=\int_{\mathfrak{a}^{F}} d \lambda^{F}\left\{\boldsymbol{\pi}^{F}\left(\frac{H^{F}}{2 t}-i \lambda_{F}-i \lambda^{F}\right)-\boldsymbol{\pi}^{F}\left(\frac{H^{F}}{2 t}\right)\right\} \\
\times \mathbf{b}^{F}\left(-\lambda_{F}-\lambda^{F}-i \frac{H^{F}}{2 t}\right)^{-1} e^{-t\left|\lambda^{F}\right|^{2}} .
\end{gathered}
$$

Using the induction hypothesis, together with $\mathbf{c}^{F}\left(-i \frac{H^{F}}{2 t}\right)^{-1} \asymp \boldsymbol{\pi}^{F}\left(\frac{H^{F}}{t}\right)$, we obtain the desired estimate for the leading term:

$$
I_{0}^{F} \geq C t^{-\frac{\ell}{2}-\mid \Sigma^{++}} \mid\left\{\prod_{\alpha \in \Sigma_{F}^{++}}(1+\langle\alpha, H\rangle)\right\} \boldsymbol{\pi}^{F}\left(H^{F}\right) e^{-|\varrho|^{2} t-\langle\varrho, H\rangle-\frac{|H|^{2}}{4 t}},
$$

with a positive constant $C$ depending possibly on $\kappa_{2}$ and on a lower bound for $\kappa_{1}$, but not on $\delta, \varepsilon$ or $N$. The other terms $I I_{0}^{F}$ and $I I I_{0}^{F}$ are comparatively smaller, as we shall see next by applying Proposition 3.9. On one hand

$$
\begin{aligned}
& \sup _{\left|\operatorname{Im} \lambda_{F}\right| \leq \frac{\left|H_{F}\right|}{2 t}}\left|\Omega_{F ; t, H}^{\prime \prime}\left(\lambda_{F}\right)\right| e^{-\frac{t}{2}\left|\operatorname{Re} \lambda_{F}\right|^{2}} \\
& \leq\left\{\int_{\mathfrak{a}^{F}} d \lambda^{F} e^{-\frac{t}{2}\left|\lambda^{F}\right|^{2}}\right\}_{|\operatorname{Im} \lambda| \leq \frac{\left|H_{F}\right|}{2 t}}\left|\mathbf{b}^{F}\left(-\lambda-i \frac{H^{F}}{2 t}\right)^{-1}-\mathbf{b}^{F}\left(-i \frac{H^{F}}{2 t}\right)^{-1}\right| e^{-\frac{t}{2}|\operatorname{Re} \lambda|^{2}} \\
& \leq C\left\{\delta+t^{-1 / 2}\right\} t^{-\ell^{F} / 2}, \\
& \text { since } \\
& \left|\mathbf{b}^{F}\left(-\lambda-i \frac{H^{F}}{2 t}\right)^{-1}-\mathbf{b}^{F}\left(-i \frac{H^{F}}{2 t}\right)^{-1}\right| \leq|\lambda| \sup _{\lambda^{\prime} \in[0, \lambda]}\left|\nabla_{\lambda^{\prime}} \mathbf{b}^{F}\left(-\lambda^{\prime}-i \frac{H^{F}}{2 t}\right)^{-1}\right| \\
& \leq C\{|\operatorname{Re} \lambda|+|\operatorname{Im} \lambda|\}(1+|\operatorname{Re} \lambda|)^{m / 2}
\end{aligned}
$$

and $|\operatorname{Re} \lambda| \leq \frac{\sqrt{t}|\operatorname{Re} \lambda|}{\sqrt{t}},|\operatorname{Im} \lambda| \leq \frac{\left|H_{F}\right|}{2 t} \leq \frac{c_{1} \delta|H|}{2 t} \leq \frac{c_{1} \kappa_{2}}{2} \delta$. Hence

$$
\left|I I_{0}^{F}\right| \leq C\left\{\delta+t^{-\frac{1}{2}}\right\} t^{-\frac{\ell}{2}-\mid \Sigma^{++}} \mid\left\{\prod_{\alpha \in \Sigma_{F}^{++}}(1+\langle\alpha, H\rangle)\right\} \boldsymbol{\pi}^{F}\left(H^{F}\right) e^{-|\varrho|^{2} t-\langle\varrho, H\rangle-\frac{|H|^{2}}{4 t}}
$$


with a constant $C$ as above. On the other hand

$$
\begin{aligned}
& \quad \sup _{\left|\operatorname{Im} \lambda_{F}\right| \leq \frac{\left|H_{F}\right|}{2 t}}\left|\Omega_{F ; t, H}^{\prime \prime \prime}\left(\lambda_{F}\right)\right| e^{-\frac{t}{2}\left|\operatorname{Re} \lambda_{F}\right|^{2}} \\
& \leq\left\{\int_{\mathfrak{a}^{F}} d \lambda^{F} e^{-\frac{t}{2}\left|\lambda^{F}\right|^{2}}\right\} \\
& \quad \times \sup _{|\operatorname{Im} \lambda| \leq \frac{\left|H_{F}\right|}{2 t}}\left|\boldsymbol{\pi}^{F}\left(\frac{H^{F}}{2 t}-i \lambda\right)-\boldsymbol{\pi}^{F}\left(\frac{H^{F}}{2 t}\right)\right|\left|\mathbf{b}^{F}\left(-\lambda-i \frac{H^{F}}{2 t}\right)\right|^{-1} e^{-\frac{t}{2}|\operatorname{Re} \lambda|^{2}} \\
& \quad \leq C\left\{\frac{\delta}{\varepsilon}+\frac{1}{\kappa_{1} \varepsilon}\right\} t^{-\frac{\ell^{F}}{2}-\left|\Sigma^{++}\right|+\left|\Sigma_{F}^{++}\right|} \boldsymbol{\pi}^{F}\left(H^{F}\right),
\end{aligned}
$$

since

$$
\begin{gathered}
\mathbf{b}^{F}\left(-\lambda-i \frac{H^{F}}{2 t}\right)^{-1}=\mathrm{O}\left((1+|\operatorname{Re} \lambda|)^{\frac{m}{2}}\right) \\
|\langle\alpha, \operatorname{Im} \lambda\rangle| \leq|\operatorname{Im} \lambda| \leq \frac{\left|H_{F}\right|}{2 t} \leq \frac{c_{1}}{2} \frac{\delta}{\varepsilon} \frac{\varepsilon|H|}{t} \leq \frac{c_{1}}{2} \frac{\delta}{\varepsilon} \frac{\omega^{F}(H)}{t} \leq \frac{c_{1}}{2} \frac{\delta}{\varepsilon} \frac{\left\langle\alpha, H^{F}\right\rangle}{t}
\end{gathered}
$$

for every $\alpha \in \Sigma^{++} \backslash \Sigma_{F}^{++}$, and similarly

$$
|\langle\alpha, \operatorname{Re} \lambda\rangle| \leq|\operatorname{Re} \lambda| \leq \frac{1}{\kappa_{1} \varepsilon} \frac{\varepsilon|H|}{t} \sqrt{t}|\operatorname{Re} \lambda| \leq \frac{1}{\kappa_{1} \varepsilon} \frac{\left\langle\alpha, H^{F}\right\rangle}{t} \sqrt{t}|\operatorname{Re} \lambda| .
$$

Hence

$$
\left|I I I_{0}^{F}\right| \leq C\left\{\frac{\delta}{\varepsilon}+\frac{1}{\kappa_{1} \varepsilon}\right\} t^{-\frac{\ell}{2}-\mid \Sigma^{++}} \mid\left\{\prod_{\alpha \in \Sigma_{F}^{++}}(1+\langle\alpha, H\rangle)\right\} \boldsymbol{\pi}^{F}\left(H^{F}\right) e^{-|\varrho|^{2} t-\langle\varrho, H\rangle-\frac{|H|^{2}}{4 t}}
$$

with $C$ as above again. Thus $I_{0}^{F}$ is the leading term in (3.39), as claimed, and we have

hence

$$
\left|I I_{0}^{F}\right|+\left|I I I_{0}^{F}\right| \leq \frac{1}{2} I_{0}^{F}
$$

$$
E_{0}^{F} \geq \frac{1}{2} I_{0}^{F}
$$

provided $\delta, \delta / \varepsilon, 1 / \kappa_{1} \varepsilon$ and $t^{-1 / 2}$ are sufficiently small, which can be achieved by choosing $\delta \varepsilon$ appropriately (see Lemma 2.1.7.ii) and by taking $\kappa_{1}$ sufficiently large. The lower estimate (3.36) follows eventually from (3.37), (3.38), (3.40), (3.41) and from

$$
\boldsymbol{\pi}^{F}\left(H^{F}\right) \asymp \prod_{\alpha \in \Sigma^{++} \backslash \Sigma_{F}^{++}}(1+\langle\alpha, H\rangle)
$$

see (3.33). This concludes Step 8.

Our heat kernel estimates are finally obtained by putting together all cases dealt with in the previous steps.

REMARK. This is the second application known as yet of the Trombi \& Varadarajan expansion for spherical functions (Theorem 2.2.8). Recall that the first one in $[\mathrm{TrV}]$ dealt with the spherical Fourier transform of the $L^{p}$ Schwartz spaces $\mathcal{S}^{p}(G)^{\natural}$, and that this result was later on obtained 
in a much simpler way [An4]. Here too the recourse to the Trombi \& Varadarajan expansion could be avoided, as we realized lately [AnJ2]. One uses instead the parabolic Harnack inequality of Li \& Yau ([LiY], see also [Da, Section 5.3]), which allows to shift heat kernel estimates away from the walls. Of course this simplification works only for bounds and not for asymptotics (subsection 5.1). Besides we think that the delicate analysis carried out in this section is interesting in its own and may be helpful for other problems.

\section{Some Applications}

4.1 Heat propagation. In this subsection we shall assume that $\mathbb{X}=$ $G / K$ has no Euclidean factor. (Actually all results for $1 \leq p \leq 2$ hold provided $\mathbb{X}$ is not trivially Euclidean.) Recall that $h_{t}$ denotes the heat kernel on $\mathbb{X}$. The behavior of its $L^{p}$-norm $\left\|h_{t}\right\|_{p}$ can be easily deduced from the pointwise estimates established or recalled in Section 3, specifically Theorem 3.7.ii and (3.3). This result was obtained in a more abstract way in [CowGM1].

Proposition 4.1.1. (i) For $t$ small,

$$
\left\|h_{t}\right\|_{p} \asymp t^{-n / 2 p^{\prime}} \quad \forall 1 \leq p \leq+\infty,
$$

where $p^{\prime}=\frac{p}{p-1}$ denotes the dual index.

(ii) For $t$ large,

$$
\left\|h_{t}\right\|_{p} \asymp \begin{cases}t^{-\frac{\ell}{2 p^{\prime}}} e^{-\frac{4}{p p^{\prime}}|\varrho|^{2} t} & \text { if } 1 \leq p<2, \\ t^{-\frac{\ell}{4}-\frac{\left|\Sigma^{++}\right|}{2}} e^{-|\varrho|^{2} t} & \text { if } \quad p=2, \\ t^{-\frac{\ell}{2}-\mid \Sigma^{++}} e^{-|\varrho|^{2} t} & \text { if } 2<p \leq+\infty .\end{cases}
$$

Davies [Da, Corollary 5.7.3] observed that heat diffusion on real hyperbolic spaces propagates asymptotically with finite speed. This striking phenomenon was later on extended to noncompact symmetric spaces, by analytic means in [AnSe] and probabilistic ones in [B2]. It reflects the interplay between the heat kernel decay on one hand and the volume growth on the other hand. This becomes more apparent if we go beyond $L^{1}$ and consider the general $L^{p}$ setting.

Theorem 4.1.2. (i) Case $1 \leq p<2$ : For $t$ large, consider the box

$$
B_{p}(t)=\left\{H \in \mathfrak{a}|2| \varrho_{p}|t-r(t) \leq| H|\leq 2| \varrho_{p} \mid t+r(t), \varangle(H, \varrho) \leq \theta(t)\right\}
$$

centered at the point $2 t \varrho_{p}=4\left(\frac{1}{p}-\frac{1}{2}\right)$ t@ in $\mathfrak{a}$, where $r(t)$ and $\theta(t)$ are positive 
functions such that

$$
\left\{\begin{array} { l } 
{ \frac { r ( t ) } { t } \rightarrow 0 } \\
{ \frac { r ( t ) } { \sqrt { t } } \rightarrow + \infty }
\end{array} \quad \text { and } \quad \left\{\begin{array}{l}
\theta(t) \rightarrow 0 \\
\sqrt{t} \theta(t) \rightarrow+\infty
\end{array} \quad \text { as } t \rightarrow+\infty .\right.\right.
$$

Then the $L^{p}$ norm of $h_{t}$ concentrates asymptotically in the bi- $K$-orbit of $\exp B_{p}(t)$ :

$$
\left\|h_{t}\right\|_{p}^{-1}\left\{\int_{G \backslash K\left\{\exp B_{p}(t)\right\} K} d x\left|h_{t}(x)\right|^{p}\right\}^{1 / p} \longrightarrow 0 \quad \text { as } t \rightarrow+\infty .
$$

(ii) Case $p=2$ : We have a similar concentration

with respect to boxes

$$
\left\|h_{t}\right\|_{2}^{-1}\left\{\int_{G \backslash K\left\{\exp B_{2}(t)\right\} K} d x\left|h_{t}(x)\right|^{2}\right\}^{1 / 2} \longrightarrow 0
$$

where

$$
B_{2}(t)=\left\{H \in \mathfrak{a}\left|r_{1}(t) \leq\right| H \mid \leq r_{2}(t), \omega(H) \geq r_{3}(t)\right\},
$$

$$
\left\{\begin{array} { l } 
{ r _ { 1 } ( t ) \rightarrow + \infty } \\
{ \frac { r _ { 1 } ( t ) } { \sqrt { t } } \rightarrow 0 }
\end{array} \quad , \quad \frac { r _ { 2 } ( t ) } { \sqrt { t } } \rightarrow + \infty \quad \text { and } \quad \left\{\begin{array}{l}
r_{3}(t) \rightarrow+\infty \\
\frac{r_{3}(t)}{\sqrt{t}} \rightarrow 0
\end{array}\right.\right.
$$

(iii) Case $2<p<+\infty$ :

$$
\left\|h_{t}\right\|_{p}^{-1}\left\{\int_{|x| \geq r(t)} d x\left|h_{t}(x)\right|^{p}\right\}^{1 / p} \longrightarrow 0
$$

if $r(t) \rightarrow+\infty$ as $t \rightarrow+\infty$.

(iv) Case $p=+\infty: h_{t}(x)$ reaches its maximum at $x=e$ and

$$
\left\|h_{t}\right\|_{\infty}^{-1} \sup _{|x| \geq r(t)} h_{t}(x) \longrightarrow 0
$$

again if $r(t) \rightarrow+\infty$.

Notice that $p=2$ is the only case which resembles the Euclidean setting. Sketch of proof. As a positive-definite function, $h_{t}=h_{t / 2} * h_{t / 2}^{\vee}$ reaches its maximum at the origin. The rest of the proof is similar to the $L^{1}$ case treated in [AnSe, Section 2]. It relies on the integral formula (2.1.5) for bi- $K$-invariant functions:

$$
\int_{G} d x f(x)=\text { const. } \int_{\mathfrak{a}^{+}} d H \boldsymbol{\delta}(H) f(\exp H) \quad \text { with } \quad \delta(H)=\mathrm{O}\left(e^{2\langle\varrho, H\rangle}\right),
$$
and on the upper bounds in Theorem 3.7.ii for $t$ large (and $|H|=\mathrm{O}(t)$ ):

$$
h_{t}(\exp H) \leq C t^{-\frac{\ell}{2}-\mid \Sigma^{++}} \mid\left\{\prod_{\alpha \in \Sigma^{++}}(1+\langle\alpha, H\rangle)\right\} e^{-|\varrho|^{2} t-\langle\varrho, H\rangle-\frac{|H|^{2}}{4 t}},
$$

and in (3.3) for $|H| \gg t$ large (or any other Gaussian upper estimate):

$$
h_{t}(\exp H) \leq C|H|^{d} e^{-|\varrho|^{2} t-\langle\varrho, H\rangle-\frac{|H|^{2}}{4 t}} .
$$


4.2 Bessel-Green-Riesz kernels. Let $\Delta$ be the Laplace-Beltrami operator on $\mathbb{X}=G / K$. Recall that its $L^{2}$-spectrum consists of the half-line $\left(-\infty,-|\varrho|^{2}\right]$ and that its spectral decomposition is given by

$$
E\left(\left[-|\varrho|^{2}-R_{2}^{2},-|\varrho|^{2}-R_{1}^{2}\right]\right) f=\frac{c_{2}}{|W|} \int_{\left\{\lambda \in \mathfrak{a}\left|R_{1} \leq\right| \lambda \mid \leq R_{2}\right\}} \frac{d \lambda}{|\mathbf{c}(\lambda)|^{2}} f * \varphi_{\lambda} .
$$

The Bessel-Green-Riesz potentials are the following spectrally defined functions of $\Delta$ :

$$
\left(-\Delta-|\varrho|^{2}+\zeta^{2}\right)^{-\sigma / 2} \quad \forall \zeta \in(0,+\infty)+i \mathbb{R}, \forall \sigma \in \mathbb{C} .
$$

Their (Schwartz) kernel will be denoted by $k_{\zeta, \sigma}$, and simply by $k_{\zeta}$ when $\sigma=2$ (Green function).

These expressions extend to the limit case $\zeta=0$ provided $\sigma \notin \ell+$ $2\left|\Sigma^{++}\right|+2 \mathbb{N}$ [CowGM1, Section 6]. Notice that the first singularity $\sigma=$ $\ell+2\left|\Sigma^{++}\right|$is related to the vanishing of the spectral measure (4.2.1) at $-|\varrho|^{2}$, i.e. of the Plancherel measure at the origin.

Theorem 4.2.2. (i) Let $\zeta>0, \sigma>0$ and $D \in \mathrm{U}(\mathfrak{g})$. Then

and

$$
k_{\zeta, \sigma}(x) \asymp|x|^{\frac{\sigma-\ell-1}{2}-\left|\Sigma^{++}\right|} \varphi_{0}(x) e^{-\zeta|x|}
$$

away from the origin.

$$
k_{\zeta, \sigma}(x: D)=\mathrm{O}\left(k_{\zeta, \sigma}(x)\right)
$$

(ii) In the limit case $\zeta=0$ and for $0<\sigma<\ell+2\left|\Sigma^{++}\right|$, we have instead

$$
k_{0, \sigma}(x) \asymp|x|^{\sigma-\ell-2 \mid \Sigma^{++}} \varphi_{0}(x) .
$$

REMARK 4.2.3. (i) Recall the behavior of $\varphi_{0}$ (Proposition 2.2.12.i):

$$
\varphi_{0}(\exp H) \asymp\left\{\prod_{\alpha \in \Sigma^{++}}(1+\langle\alpha, H\rangle)\right\} e^{-\langle\varrho, H\rangle} \quad \forall H \in \overline{\mathfrak{a}^{+}} .
$$

(ii) Notice the jump in the polynomial factor between the cases $\zeta>0$ and $\zeta=0$.

(iii) The behavior of $k_{\zeta, \sigma}$ at the origin is classical and well known:

$$
k_{\zeta, \sigma}(x) \asymp\left\{\begin{array}{cl}
|x|^{\sigma-n} & \text { if } 0<\sigma<n \\
\log \frac{1}{|x|} & \text { if } \sigma=n \\
1 & \text { if } \sigma>n
\end{array}\right.
$$

and

$$
k_{\zeta, \sigma}(x: D)= \begin{cases}\mathrm{O}\left(|x|^{\sigma-n-\operatorname{deg} D}\right) & \text { if } 0<\sigma<n+\operatorname{deg} D \\ \mathrm{O}\left(\log \frac{1}{|x|}\right) & \text { if } \sigma=n+\operatorname{deg} D \\ \mathrm{O}(1) & \text { if } \sigma>n+\operatorname{deg} D\end{cases}
$$

with the restriction $0<\sigma<\ell+2\left|\Sigma^{++}\right|$if $\zeta=0$. 
(iv) As mentioned in the introduction, the Green function estimates

$$
k_{\zeta}(x) \asymp \begin{cases}|x|^{\frac{1-\ell}{2}-\left|\Sigma^{++}\right|} e^{-\zeta|x|} \varphi_{0}(x) & \text { if } \zeta>0 \\ |x|^{2-\ell-2 \mid \Sigma^{++}} \varphi_{0}(x) & \text { if } \zeta=0\end{cases}
$$

away from the origin (especially when $\zeta>0$ ) constitute the key analytic ingredient in the determination of the Martin compactification of $\mathbb{X}=G / K$ $([\mathrm{Gu}],[\mathrm{GuJT} 1,2])$. They play a further role in the study of PattersonSullivan measures on $\mathbb{X}[\mathrm{A}]$.

Proof of Theorem 4.2.2. Recall the Mellin type expression

$$
\left(-\Delta-|\varrho|^{2}+\zeta^{2}\right)^{-\frac{\sigma}{2}}=\frac{1}{\Gamma\left(\frac{\sigma}{2}\right)} \int_{0}^{+\infty} \frac{d t}{t} t^{\frac{\sigma}{2}} e^{\left(|\varrho|^{2}-\zeta^{2}\right) t} e^{t \Delta}
$$

of the Bessel-Green-Riesz potentials in terms of the heat semigroup $e^{t \Delta}$, which writes

$$
k_{\zeta, \sigma}(x)=\frac{1}{\Gamma\left(\frac{\sigma}{2}\right)} \int_{0}^{+\infty} \frac{d t}{t} t^{\frac{\sigma}{2}} e^{\left(|\varrho|^{2}-\zeta^{2}\right) t} h_{t}(x)
$$

on the kernel level.

(i) When $\zeta>0$, the main contribution in (4.2.4.2) comes from the integral where $t \sim|x| / 2 \zeta$. Thus let us split

$$
\int_{0}^{+\infty}=\int_{0}^{\kappa^{-1} \frac{|x|}{2 \zeta}}+\int_{\kappa^{-1} \frac{|x|}{2 \zeta}}^{\kappa \frac{|x|}{2 \zeta}}+\int_{\kappa \frac{|x|}{2 \zeta}}^{+\infty}
$$

for some $\kappa>1$. According to Theorem 3.7.ii, the second integral is comparable to

hence to

$$
|x|^{\frac{\sigma-\ell}{2}-\left|\Sigma^{++}\right|-1} \varphi_{0}(x) \int_{\kappa^{-1} \frac{|x|}{2 \zeta}}^{\kappa \frac{|x|}{2 \zeta}} d t e^{-\zeta^{2} t-\frac{|x|^{2}}{4 t}}
$$

since

$$
|x|^{\frac{\sigma-\ell-1}{2}-\left|\Sigma^{++}\right|} \varphi_{0}(x) e^{-\zeta|x|},
$$

$$
\int_{\kappa^{-1} \frac{|x|}{2 \zeta}}^{\kappa \frac{|x|}{2 \zeta}} d t e^{-\zeta^{2} t-\frac{|x|^{2}}{4 t}}=\frac{|x|}{2 \zeta} \int_{\kappa^{-1}}^{\kappa} d t e^{-\zeta|x| \frac{t+t^{-1}}{2}} \sim \sqrt{\frac{\pi}{2}} \zeta^{-3 / 2}|x|^{\frac{1}{2}} e^{-\zeta|x|}
$$

by an elementary application of the Laplace method (see for instance [Co, Ch. 5]). Similarly, the third integral is bounded by

$$
C \varphi_{0}(x) \int_{\kappa \frac{|x|}{2 \zeta}}^{+\infty} d t t^{\frac{\sigma-\ell}{2}-\left|\Sigma^{++}\right|-1} e^{-\zeta^{2} t-\frac{|x|^{2}}{4 t}}
$$

which is a $\mathrm{O}\left(\varphi_{0}(x) e^{-(\zeta+\eta)|x|}\right)$ for some $\eta>0$ depending on $\zeta, \sigma$ and $\kappa$. For the first one, we use instead of Theorem 3.7 any heat kernel Gaussian 
estimate, for instance the result (3.3) borrowed from [An5], and obtain as an upper bound

$$
C|x|^{d} \varphi_{0}(x) \int_{0}^{\kappa^{-1} \frac{|x|}{2 \zeta}} \frac{d t}{t} t^{\frac{\sigma}{2}} e^{-\zeta^{2} t-\frac{|x|^{2}}{4 t}}
$$

which is also a $\mathrm{O}\left(\varphi_{0}(x) e^{-(\zeta+\eta)|x|}\right)$. This establishes the expected behavior of $\kappa_{\zeta, \sigma}$. Derivatives are estimated in the same way.

(ii) When $\zeta=0$, the main contribution in (4.2.4.2) comes instead from the integral where $t \asymp|x|^{2}$. Let $\eta$ be a small positive constant. On one hand, using Theorem 3.7.ii,

with

$$
\int_{|x|^{2-\eta}}^{+\infty} \frac{d t}{t} t^{\frac{\sigma}{2}} e^{|\varrho|^{2} t} h_{t}(x) \asymp \varphi_{0}(x) \int_{|x|^{2-\eta}}^{+\infty} \frac{d t}{t} t^{\frac{\sigma-\ell}{2}-\left|\Sigma^{++}\right|} e^{-\frac{|x|^{2}}{4 t}}
$$

$$
\int_{|x|^{2-\eta}}^{+\infty} \frac{d t}{t} t^{\frac{\sigma-\ell}{2}-\left|\Sigma^{++}\right|} e^{-\frac{|x|^{2}}{4 t}} \sim \Gamma\left(\frac{\ell-\sigma}{2}+\left|\Sigma^{++}\right|\right)\left(\frac{|x|}{2}\right)^{\sigma-\ell-2\left|\Sigma^{++}\right|} .
$$

On the other hand, using (3.3),

with

$$
\int_{0}^{|x|^{2-\eta}} \frac{d t}{t} t^{\frac{\sigma}{2}} e^{|\varrho|^{2} t} h_{t}(x) \leq C|x|^{d} \varphi_{0}(x) \int_{0}^{|x|^{2-\eta}} \frac{d t}{t} t^{\frac{\sigma}{2}} e^{-\frac{|x|^{2}}{4 t}}
$$

Derivatives of $k_{0, \sigma}$ are estimated in the same way.

$$
\int_{0}^{|x|^{2-\eta}} \frac{d t}{t} t^{\frac{\sigma}{2}} e^{-\frac{|x|^{2}}{4 t}}=\mathrm{O}\left(|x|^{-\infty}\right)
$$

4.3 Poisson kernel. Beside the heat semigroup $e^{t \Delta}$, another classical semigroup in harmonic analysis is the Poisson semigroup $e^{-t \sqrt{-\Delta}}$. It has been studied in various settings, including noncompact symmetric spaces (see for instance [An5], [AnDY], [CowGM2], [Lo1-5], [St]). In this subsection we shall determine the behavior of its kernel $p_{t}(x)$. Actually we shall consider a slightly more general expression, namely the kernel $p_{t, \zeta}(x)$ of $e^{-t \sqrt{-\Delta-|\varrho|^{2}+\zeta^{2}}}$, where $\zeta \geq 0$.

Theorem 4.3.1. (i) Assume $\zeta>0$. Then

$$
p_{t, \zeta}(x) \asymp \begin{cases}t(t+|x|)^{-n-1} & \text { if } t+|x| \leq 1 \\ t(t+|x|)^{-\frac{\ell}{2}-\left|\Sigma^{++}\right|-1} \varphi_{0}(x) e^{-\zeta \sqrt{t^{2}+|x|^{2}}} & \text { if } t+|x| \geq 1\end{cases}
$$

for $t>0$ and $x \in G$. Moreover

$$
\left\{\begin{aligned}
p_{t, \zeta}(x: D) & =\mathrm{O}\left(\min \{1, t+|x|\}^{-\operatorname{deg} D} p_{t, \zeta}(x)\right) \\
\frac{\partial}{\partial t} p_{t, \zeta}(x: D) & =\mathrm{O}\left(\min \left\{t, 1+\frac{|x|}{t}\right\}^{-1} \min \{1, t+|x|\}^{-\operatorname{deg} D} p_{t, \zeta}(x)\right)
\end{aligned}\right.
$$

for every $D \in \mathrm{U}(\mathfrak{g})$. 
(ii) In the limit case $\zeta=0$, we have instead

$$
p_{t, 0}(x) \asymp \begin{cases}t(t+|x|)^{-n-1} & \text { if } t+|x| \leq 1 \\ t(t+|x|)^{-\ell-2\left|\Sigma^{++}\right|-1} \varphi_{0}(x) & \text { if } \quad t+|x| \geq 1\end{cases}
$$

and

$$
\left\{\begin{array}{l}
p_{t, 0}(x: D)=\mathrm{O}\left(\min \{1, t+|x|\}^{-\operatorname{deg} D} p_{t, 0}(x)\right) \\
\frac{\partial}{\partial t} p_{t, 0}(x: D)=\mathrm{O}\left(t^{-1} \min \{1, t+|x|\}^{-\operatorname{deg} D} p_{t, 0}(x)\right)
\end{array}\right.
$$

Remarks. (i) Recall again the behavior of $\varphi_{0}$ (Proposition 2.2.12.i):

$$
\varphi_{0}(\exp H) \asymp\left\{\prod_{\alpha \in \Sigma^{++}}(1+\langle\alpha, H\rangle)\right\} e^{-\langle\varrho, H\rangle} \quad \forall H \in \overline{\mathfrak{a}^{+}} .
$$

(ii) Notice again the jump in the polynomial factors between the cases $\zeta>0$ and $\zeta=0$.

Proof of Theorem 4.3.1. Theorem 4.3.1 is proved like Theorem 4.2.2. Instead of (4.2.4) we use the subordination formula

$$
e^{-t \sqrt{-\Delta-|\varrho|^{2}+\zeta^{2}}}=\frac{t}{2 \sqrt{\pi}} \int_{0}^{+\infty} d s s^{-\frac{3}{2}} e^{-\frac{t^{2}}{4 s}} e^{-s\left(-\Delta-|\varrho|^{2}+\zeta^{2}\right)}
$$

which writes

$$
p_{t, \zeta}(x)=\frac{t}{2 \sqrt{\pi}} \int_{0}^{+\infty} d s s^{-\frac{3}{2}} e^{\left(|\varrho|^{2}-\zeta^{2}\right) s} e^{-\frac{t^{2}}{4 s}} h_{s}(x)
$$

on the kernel level. Assume first $\zeta>0$ and $t+|H| \geq 1$. In (4.3.2.2) the main contribution comes from the integral where $s \sim \sqrt{t^{2}+|x|^{2}} / 2 \zeta$. Thus let us split

$$
\int_{0}^{+\infty}=\int_{0}^{\kappa^{-1} \frac{\sqrt{t^{2}+|x|^{2}}}{2 \zeta}}+\int_{\kappa^{-1}}^{\kappa \frac{\sqrt{t^{2}+|x|^{2}}}{2 \zeta}}+\int_{\kappa}^{+\infty} \frac{\sqrt{t^{2}+|x|^{2}}}{2 \zeta}
$$

for some $\kappa>1$. According to the heat kernel estimate in Theorem 3.7.ii, the second integral is comparable to

$$
t(t+|x|)^{-\frac{\ell+3}{2}-\left|\Sigma^{++}\right|} \varphi_{0}(x) \int_{\kappa^{-1}}^{\kappa \frac{\sqrt{t^{2}+|x|^{2}}}{2 \zeta}} \frac{\sqrt{t^{2}+|x|^{2}}}{2 \zeta} d s e^{-\zeta^{2} s} e^{-\frac{t^{2}+|x|^{2}}{4 s}},
$$

hence to

$$
(t+|x|)^{-\frac{\ell}{2}-\left|\Sigma^{++}\right|-1} \varphi_{0}(x) e^{-\zeta \sqrt{t^{2}+|x|^{2}}},
$$

since

$$
\begin{aligned}
& \int_{\kappa^{-1}}^{\kappa \frac{\sqrt{t^{2}+|x|^{2}}}{2 \zeta}} \frac{\sqrt{t^{2}+|x|^{2}}}{2 \zeta} d s e^{-\zeta^{2} s} e^{-\frac{t^{2}+|x|^{2}}{4 s}}=\frac{\sqrt{t^{2}+|x|^{2}}}{2 \zeta} \int_{\kappa^{-1}}^{\kappa} d s e^{-\frac{\zeta}{2} \sqrt{t^{2}+|x|^{2}}\left(s+s^{-1}\right)} \\
& \sim \sqrt{\frac{\pi}{2}} \zeta^{-\frac{3}{2}}\left(t^{2}+|x|^{2}\right)^{\frac{1}{4}} e^{-\zeta \sqrt{t^{2}+|x|^{2}}}
\end{aligned}
$$


as $t+|x| \rightarrow+\infty$, again by the Laplace method [Co, Ch. 5]. Altogether

$$
\begin{aligned}
& t \int_{\kappa^{-1}}^{\kappa \frac{\sqrt{t^{2}+|x|^{2}}}{2 \zeta}} \frac{\sqrt{t^{2}+|x|^{2}}}{2 \zeta} d s s^{-\frac{3}{2}} e^{\left(|\varrho|^{2}-\zeta^{2}\right) s} e^{-\frac{t^{2}}{4 s}} h_{s}(x) \\
& \asymp t(t+|x|)^{-\frac{\ell}{2}-\left|\Sigma^{++}\right|-1} \varphi_{0}(x) e^{-\zeta \sqrt{t^{2}+|x|^{2}}} .
\end{aligned}
$$

As far as they are concerned, the first and the last integrals in (4.3.3) are

$$
\mathrm{O}\left(\varphi_{0}(x) e^{-(\zeta+\eta) \sqrt{t^{2}+|x|^{2}}}\right) \text {, }
$$

for some $\eta>0$. This is obtained by elementary computations as above, using the heat kernel upper estimates in (3.3) and in Theorem 3.7.ii .

The other cases are simpler. When $\zeta=0$ and $t+|x| \geq 1$, the main contribution in (4.3.2.2) comes from the integral where $s \asymp t^{2}+|x|^{2}$. We split therefore

$$
\int_{0}^{+\infty}=\int_{0}^{\left(t^{2}+|x|^{2}\right)^{1-\eta}}+\int_{\left(t^{2}+|x|^{2}\right)^{1-\eta}}^{+\infty}
$$

with $\eta>0$ small. The second integral is then comparable to

$$
t(t+|x|)^{-\ell-2\left|\Sigma^{++}\right|-1} \varphi_{0}(x)
$$

while the first one is $\mathrm{O}\left(t(t+|x|)^{-\infty} \varphi_{0}(x)\right)$. Derivatives are estimated in the same way.

4.4 Heat maximal operator on $S=(\exp \mathfrak{a}) \boldsymbol{N}$. Recall that the Laplace-Beltrami operator $\Delta$ on $\mathbb{X}=G / K$ is defined by (the left or right action of) the Casimir operator $\Omega \in \mathrm{U}(\mathfrak{g})$ :

$$
\Delta f(x K)=f(\Omega: x)=f(x: \Omega) \quad \forall x \in G .
$$

Given bases $\left\{H_{i}\right\}$ of $\mathfrak{a},\left\{Z_{j}\right\}$ of $\mathfrak{m}$, and $\left\{X_{k}\right\}$ of $\mathfrak{n}$, which are orthonormal with respect to the inner product (2.1.1)) and the latter basis being compatible with the root space decomposition $\mathfrak{n}=\oplus_{\alpha \in \Sigma^{+}} \mathfrak{g}_{\alpha}$, we have

$$
\begin{aligned}
\Omega & =\sum_{i} H_{i}^{2}-\sum_{j} Z_{j}^{2}-\sum_{k}\left\{X_{k} \theta\left(X_{k}\right)+\theta\left(X_{k}\right) X_{k}\right\} \\
& \equiv \sum_{i} H_{i}^{2}-2 \varrho+2 \sum_{k} X_{k}^{2} \quad \text { modulo } \mathrm{U}(\mathfrak{g}) \mathfrak{k} .
\end{aligned}
$$

Thus (4.4.1) rewrites

$$
\Delta f(x K)=f\left(x: \sum_{i} H_{i}^{2}-2 \varrho+2 \sum_{k} X_{k}^{2}\right) \quad \forall x \in G .
$$

Owing to the Iwasawa decomposition $G=S K, \mathbb{X}=G / K$ can be identified with the solvable Lie group $S=(\exp \mathfrak{a}) N=N(\exp \mathfrak{a})$, equipped with the left-invariant Riemannian structure induced by

$$
\left(H+X, H^{\prime}+X^{\prime}\right) \longmapsto\left\langle H, H^{\prime}\right\rangle+\frac{1}{2}\left\langle X, X^{\prime}\right\rangle
$$


for every $H+X$ and $H^{\prime}+X^{\prime}$ in $\mathfrak{s}=\mathfrak{a} \oplus \mathfrak{n}$. Instead of the Laplace-Beltrami operator (4.4.2), consider the following left-invariant differential operator on $S$ :

$$
\widetilde{\Delta} f(x)=f(x: \widetilde{\Omega}) \quad \forall x \in S, \quad \text { where } \quad \widetilde{\Omega}=\sum_{i} H_{i}^{2}+2 \sum_{k} X_{k}^{2} \in \mathrm{U}(\mathfrak{s}) .
$$

This distinguished Laplacian is (essentially) self-adjoint with respect to the right-invariant Haar measure

$$
\int_{S} d_{r} x f(x)=\int_{\mathfrak{a}} d H e^{2\langle\varrho, H\rangle} \int_{N} d n f((\exp H) n)=\int_{N} d n \int_{\mathfrak{a}} d H f(n \exp H),
$$

which will be our reference measure on $S$ henceforth. It has been considered earlier by several authors ([Bo3], [CowGGM], [CowGHM], [DeGav], [GaSj], [GiMau], $[\mathrm{H}],[\mathrm{Hu}],[\mathrm{Va}], \ldots)$, some of them interchanging left and right sides. For clarity let us indicate the relations between functions of $\Delta$ and $\widetilde{\Delta}$, and between the corresponding convolution kernels $k$ and $\widetilde{k}$ :

$$
\begin{aligned}
& m\left(\Delta+|\varrho|^{2}\right)=\widetilde{\delta}^{-1 / 2} \circ m(\widetilde{\Delta}) \circ \widetilde{\delta}^{1 / 2}, \\
& k\left(K x^{ \pm 1} K\right)=\widetilde{\delta}(x)^{-1 / 2} \widetilde{k}(x) \quad \forall x \in S,
\end{aligned}
$$

where $\widetilde{\delta}((\exp H) n)=\widetilde{\delta}(n \exp H)=e^{-2\langle\varrho, H\rangle}$ denotes the modular function of $S$. In particular the heat kernels $h_{t}$ and $\widetilde{h}_{t}$ associated to $\Delta$ and $\widetilde{\Delta}$ are related by

$$
\widetilde{h}_{t}(x)=\widetilde{\delta}(x)^{\frac{1}{2}} e^{|\varrho|^{2} t} h_{t}(K x K) \quad \forall x \in S .
$$

The estimates established or recalled in section 3 enable us to prove the weak type $(1,1)$ inequality for the heat maximal operator

$$
\left\{H_{*} f\right\}(x)=\sup _{t>0}\left|\left\{e^{t \widetilde{\Delta}} f\right\}(x)\right|
$$

on $S$, which was looked for in [CowGGM] and obtained there in some particular cases.

Theorem 4.4.4. There exists a positive constant $C$ such that

$$
\left|\left\{x \in S \mid H_{*} f(x)>\tau\right\}\right| \leq C \tau^{-1}\|f\|_{L^{1}} \quad \forall f \in L^{1}(S), \forall \tau>0 .
$$

Proof of Theorem 4.4.4. We shall resume the clever method pursued in [CowGGM]. By using (4.4.3), let us first transfer certain estimates of section 3 from $h_{t}$ to $\widetilde{h}_{t}$ :

$$
\begin{array}{ll}
\widetilde{h}_{t}(x) \asymp t^{-\frac{n}{2}}(1+t)^{\frac{m}{2}-\mid \Sigma^{++}} \mid \widetilde{\delta}(x)^{\frac{1}{2}} \varphi_{0}(x) e^{-\frac{|x|^{2}}{4 t}} \quad \text { if } \quad|x| \leq 1+t, \\
\widetilde{h}_{t}(x)=\mathrm{O}\left((1+|x|)^{d} \widetilde{\delta}(x)^{\frac{1}{2}} \varphi_{0}(x) e^{-\frac{|x|^{2}}{4 t}}\right) \quad \text { if } \quad|x| \geq \max \{1, t\} .
\end{array}
$$

The weak type $(1,1)$ estimate is known to hold for the local maximal function

$$
\left\{H_{*}^{0} f\right\}(x)=\sup _{0<t<1}\left|\left\{e^{t \widetilde{\Delta}} f\right\}(x)\right| .
$$


Thus we are left with the global maximal function

$$
\left\{H_{*}^{\infty} f\right\}(x)=\sup _{t \geq 1}\left|\left\{e^{t \widetilde{\Delta}} f\right\}(x)\right|,
$$

which is estimated as follows:

$$
\left\{H_{*}^{\infty} f\right\}(x) \leq\left\{|f| * \widetilde{h}_{*}\right\}(x)+\sup _{t \geq 1}\left|f *\left\{\chi_{t} \widetilde{h}_{t}\right\}(x)\right|,
$$

where $\widetilde{h}_{*}(x)=\sup _{1 \leq t \leq|x|} \widetilde{h}_{t}(x)$ and $\chi_{t}(x)$ denotes the characteristic function of the ball $|x| \leq t$ in $S$. On one hand (4.4.7) yields

$$
\widetilde{h}_{*}(x)=\mathrm{O}\left((1+|x|)^{d} \widetilde{\delta}(x)^{1 / 2} \varphi_{0}(x) e^{-|x| / 4}\right),
$$

which implies the integrability of $\widetilde{h}_{*}$. For any $\eta>0$, we have indeed

$$
\begin{aligned}
\int_{S} d_{r} x \widetilde{\delta}(x)^{\frac{1}{2}} \varphi_{0}(x) e^{-\eta|x|} & =\int_{N} d n \int_{\mathfrak{a}} d H e^{-\langle\varrho, H\rangle} \varphi_{0}(n \exp H) e^{-\eta|n \exp H|} \\
& =2^{\frac{m}{2}} \int_{G} d x e^{-\left\langle\varrho, H\left(x^{ \pm 1}\right)\right\rangle} \varphi_{0}(x) e^{-\eta|x|} \\
& =2^{\frac{m}{2}} \int_{G} d x\left\{\int_{K} d k e^{-\langle\varrho, H(x k)\rangle}\right\} \varphi_{0}(x) e^{-\eta|x|} \\
& =2^{\frac{m}{2}} \int_{G} d x \varphi_{0}(x)^{2} e^{-\eta|x|} \\
& \leq C \int_{\overline{\mathfrak{a}^{+}}} d H e^{-\frac{\eta}{2}|H|}<+\infty,
\end{aligned}
$$

using (2.1.4), (2.1.5), (2.2.1) and Proposition 2.2.12.i. As a consequence the operator $f \mapsto|f| * \widetilde{h}_{*}$ is of strong type $(1,1)$. On the other hand we deduce from (4.4.6) that

$$
\chi_{t}(x) \widetilde{h}_{t}(x) \leq C t^{-1} \int_{t}^{2 t} d s \widetilde{h}_{s}(x) \quad \forall t \geq 1, \forall x \in S,
$$

and the averaged convolution kernel yields a weak type $(1,1)$ maximal operator, according to the Hopf-Dunford-Schwartz ergodic theorem [DunSch, Ch. VIII]. Altogether $H_{*}^{\infty}$ is a weak type $(1,1)$ operator and this concludes the proof of Theorem 4.4.4.

REmarks. (i) The analogous result for the heat diffusion on $G / K$ is proved quite differently [An5].

(ii) The kernel behavior of the Bessel-Green-Riesz potentials $\left(-\widetilde{\Delta}+\zeta^{2}\right)^{-\sigma / 2}$ and of the Poisson semigroup $e^{-t \sqrt{-\widetilde{\Delta}+\zeta^{2}}}$ can be deduced similarly from the corresponding results for $\Delta$ in subsections 4.2 and 4.3 . 


\section{Asymptotics}

In this section we refine the results of section 3, subsection 4.2 and subsection 4.3 by obtaining asymptotics at infinity instead of global estimates for the heat kernel $h_{t}(\exp H)$, for the Bessel-Green-Riesz kernels $k_{\zeta, \sigma}(\exp H)$ and for the Poisson kernel $p_{t, \zeta}(\exp H)$ on $\mathbb{X}=G / K$. Such precise informations, notably the asymptotics along faces, were previously unavailable in the literature, except for some particular cases where specific expressions could be used (rank $\mathbb{X}=1, G$ complex, $G$ real normal, i.e. totally split, $G=\mathrm{SU}(p, q), G=\mathrm{SL}(n, \mathbb{H}), G=" \mathrm{SL}(3, \mathbb{O}) ", \ldots)$.

Recall the meaning of the symbols

$$
\left\{\begin{array}{llc}
x_{j} \sim y_{j} & \Longleftrightarrow & \lim _{j} \frac{x_{j}}{y_{j}}=1 \\
x_{j} \asymp y_{j} & \Longleftrightarrow & 0<\inf _{j} \frac{x_{j}}{y_{j}} \leq \sup _{j} \frac{x_{j}}{y_{j}}<+\infty
\end{array}\right.
$$

between two sequences of positive numbers.

\subsection{Heat kernel $h_{t}$.}

Theorem 5.1.1. The following asymptotics hold:

$h_{t_{j}}\left(\exp H_{j}\right) \sim c_{5} \mathbf{b}\left(-i \frac{H_{j}}{2 t_{j}}\right)^{-1} t_{j}^{-\frac{\ell}{2}-\left|\Sigma^{++}\right|} \varphi_{0}\left(\exp H_{j}\right) e^{-|\varrho|^{2} t_{j}-\frac{\left|H_{j}\right|^{2}}{4 t_{j}}}$ as $j \rightarrow+\infty$, for all sequences $t_{j} \rightarrow+\infty$ and $H_{j} \in \overline{\mathfrak{a}^{+}}$satisfying one of the following two conditions:

(i) $\left\langle\alpha, H_{j}\right\rangle \rightarrow+\infty \quad \forall \alpha \in \Sigma^{+(++)}$,

(ii) $\left|H_{j}\right|=\mathrm{O}\left(t_{j}\right)$.

Here $c_{5}=c_{2} 2^{-\left|\Sigma^{++}\right|} \pi^{\ell / 2} \boldsymbol{\pi}(\varrho) \mathbf{b}(0)^{-1}$ is a positive constant.

Remarks 5.1.2. (i) The expressions $\mathbf{b}\left(-i \frac{H_{j}}{2 t_{j}}\right)^{-1}$ and $\varphi_{0}\left(\exp H_{j}\right)$ entering these asymptotics are positive and their behavior was recalled in subsection 2.2.

(ii) We conjecture that these asymptotics hold for all sequences $t_{j} \rightarrow$ $+\infty$ and $H_{j} \in \overline{\mathfrak{a}^{+}}$, without the condition $\left|H_{j}\right|=\mathrm{O}\left(t_{j}\right)$. As in Theorem 3.7.ii, our restricted assumption comes from the Trombi-Varadarajan expansion (Theorem 2.2.8).

(iii) When $G$ is complex, spherical analysis is elementary and the heat kernel behavior is easily read off from the explicit formula $[\mathrm{G}]$

$$
\begin{gathered}
h_{t}(x)=(4 \pi t)^{-\frac{n}{2}} \varphi_{0}(x) e^{-|\varrho|^{2} t-\frac{|x|^{2}}{4 t}} \quad \forall t>0, \quad \forall x \in G, \\
\text { with } \quad \varphi_{0}(\exp H)=\prod_{\alpha \in \Sigma^{+}} \frac{\langle\alpha, H\rangle}{\sinh \langle\alpha, H\rangle} \quad \forall H \in \mathfrak{a} .
\end{gathered}
$$


(iv) Theorem 5.1.1 yields complete heat kernel asymptotics in rank 1:

$$
h_{t_{j}}\left(\exp r_{j} H_{0}\right) \sim c_{5}^{\prime} \gamma\left(\frac{\kappa}{2} \frac{r_{j}}{t_{j}}\right) t_{j}^{-\frac{3}{2}} r_{j} e^{-\frac{\varrho^{2}}{\kappa^{2}} t_{j}-\varrho r_{j}-\frac{\kappa^{2}}{4} \frac{r_{j}^{2}}{t_{j}}}
$$

as $t_{j} \rightarrow+\infty$ and $r_{j} \rightarrow+\infty$. Here $\alpha$ is the short positive root, $H_{0}$ is the generator of $\mathfrak{a}$ normalized by $\left\langle\alpha, H_{0}\right\rangle=1, \kappa=\left|H_{0}\right|=|\alpha|^{-1}=\sqrt{2 m_{\alpha}+8 m_{2 \alpha}}$, $\varrho=\frac{m_{\alpha}}{2}+m_{2 \alpha}, c_{5}^{\prime}=2^{m_{2 \alpha}-1} \pi^{-\frac{n}{2}} \kappa^{3-n}$ and

$$
\gamma(s)=\frac{\Gamma\left(s+\frac{m_{\alpha}}{2}\right)}{\Gamma(s+1)} \frac{\Gamma\left(\frac{s}{2}+\frac{\rho}{2}\right)}{\Gamma\left(\frac{s}{2}+\frac{m_{\alpha}}{4}\right)}
$$

is a positive expression when $s \geq 0$, with $\gamma(0)=2^{\frac{m_{\alpha}}{2}-1} \pi^{-\frac{1}{2}} \Gamma\left(\frac{\varrho}{2}\right) \Gamma\left(\frac{m_{\alpha}}{4}+\frac{1}{2}\right)$ and

$$
\gamma(s) \sim 2^{-\frac{m_{2 \alpha}}{2}} s^{\frac{n-3}{2}} \quad \text { as } \quad s \rightarrow+\infty .
$$

This result extends obviously to Damek-Ricci spaces (see [AnDY] and the references therein).

(v) The weak asymptotic behavior of the measures $h_{t_{j}}(x) d x$ on $\mathbb{X}=$ $G / K$, for iterated times $t_{j}=j t_{1} \rightarrow+\infty$, was determined in ([Bo1,2]), as a particular case of a central limit theorem for random walks on $G$.

The proof of Theorem 5.1.1 relies on the analysis carried out in section 3 and will take up the rest of this subsection. Let us first deal with the easy cases:

(5.1.3) As noticed in Step 0 of section 3, the Euclidean setting is elementary and we can always reduce to the semisimple one.

(5.1.4) The heat kernel asymptotics in case (i) follow immediately from Step 6 in section 3 . Notice that the convergence

$$
\frac{h_{t_{j}}\left(\exp H_{j}\right)}{\mathbf{b}\left(-i \frac{H_{j}}{2 t_{j}}\right)^{-1} t_{j}^{-\frac{\ell}{2}-\mid \Sigma^{++}} \varphi_{0}\left(\exp H_{j}\right) e^{-|\varrho|^{2} t_{j}-\left(\left|H_{j}\right|^{2} / 4 t_{j}\right)}} \longrightarrow c_{5}
$$

depends only on the rate $\omega\left(H_{j}\right)=\min _{\alpha \in \Sigma^{+(++)}}\left\langle\alpha, H_{j}\right\rangle$ tends to $+\infty$. (5.1.5) The heat kernel asymptotics when $\left|H_{j}\right|=\mathrm{o}\left(\sqrt{t_{j}}\right)$ follow similarly from Step 5 in section 3 and from the formula

$$
\int_{\mathfrak{a}} d \lambda \boldsymbol{\pi}(\lambda)^{2} e^{-|\lambda|^{2}}=2^{-\left|\Sigma^{++}\right|} \pi^{\frac{\ell}{2}}|W| \boldsymbol{\pi}(\widetilde{\varrho}),
$$

which is obtained by applying $\left.\boldsymbol{\pi}\left(\frac{\partial}{\partial H}\right)^{2}\right|_{H=0}$ to $\int_{\mathfrak{a}} d \lambda e^{-|\lambda|^{2}} e^{i\langle\lambda, H\rangle}=$ $\pi^{\frac{\ell}{2}} e^{-\frac{1}{4}|H|^{2}}$ and using $\partial(\boldsymbol{\pi}) \boldsymbol{\pi}=|W| \boldsymbol{\pi}(\widetilde{\varrho})$ (see the proof of Proposition 2.2.12.ii).

(5.1.6) Let us now consider the more general case where $\left|H_{j}\right|=\mathrm{O}\left(\sqrt{t_{j}}\right)$. Assume that the heat kernel asymptotics fail to hold for two such sequences $t_{j} \in(0,+\infty)$ and $H_{j} \in \overline{\mathfrak{a}^{+}}$. According to (5.1.5), $\left|H_{j}\right| / \sqrt{t_{j}} \nrightarrow$ 0 . By passing to subsequences, we can assume that inf $f_{j}\left|H_{j}\right| / \sqrt{t_{j}}>0$, 
hence $\left|H_{j}\right| \rightarrow+\infty$. By compactness, we can furthermore assume that $H_{j} /\left|H_{j}\right|$ tends to a unit vector $H_{\infty}$ in $\overline{\mathfrak{a}^{+}}$. Because of case (i), $H_{\infty}$ cannot lie in $\mathfrak{a}^{+}$and must therefore belong to some face $\left(\mathfrak{a}^{F}\right)^{+}$. Then Step 7 in section 3 yields the heat kernel asymptotics asymptotics

$$
\begin{aligned}
h_{t_{j}}\left(\exp H_{j}\right) & \sim c_{2} 2^{-\left|\Sigma^{++}\right|} \pi^{\frac{\ell}{2}} \boldsymbol{\pi}_{F}(\widetilde{\varrho}) \mathbf{b}(0)^{-1} \mathbf{b}_{F}(0)^{-1} \\
& \times t_{j}^{-\frac{\ell}{2}-\left|\Sigma^{++}\right|} \boldsymbol{\pi}_{F}\left(H_{j}^{F}\right) \varphi_{0}^{F}\left(\exp H_{j}\right) e^{-|\varrho|^{2} t_{j}-\left\langle\varrho^{F}, H_{j}\right\rangle-\frac{\left|H_{j}^{F}\right|^{2}}{4 t_{j}}},
\end{aligned}
$$

which agree with Theorem 5.1.1, since

$$
\left\{\begin{array}{l}
\left\langle\alpha, H_{j}\right\rangle=\mathrm{o}\left(\left|H_{j}\right|\right) \quad \forall \alpha \in F \\
\left\langle\alpha, H_{j}\right\rangle \asymp\left|H_{j}\right| \quad \forall \alpha \in \Sigma^{+++} \backslash F,
\end{array}\right.
$$

hence

$$
\begin{aligned}
& \left\langle\alpha, H_{j}\right\rangle \sim\left\langle\alpha, H_{j}^{F}\right\rangle \quad \forall \alpha \in \Sigma^{++} \backslash \Sigma_{F}^{++}, \\
& \left|\left(H_{j}\right)_{F}\right|=\mathrm{o}\left(\left|H_{j}\right|\right),
\end{aligned}
$$

and

$$
\varphi_{0}\left(\exp H_{j}\right) \sim \boldsymbol{\pi}^{F}(\widetilde{\varrho})^{-1} \mathbf{b}^{F}(0) \boldsymbol{\pi}^{F}\left(H_{j}^{F}\right) \varphi_{0}^{F}\left(\exp H_{j}\right) e^{-\left\langle\varrho^{F}, H_{j}\right\rangle}
$$

according to (2.2.13.iii). This contradicts our assumptions.

Next assume that the heat kernel asymptotics hold in case (ii) for all proper symmetric subspaces with no Euclidean factor in $\mathbb{X}$, but not for $\mathbb{X}$ itself. Consider two sequences $t_{j} \in(0,+\infty)$ and $H_{j} \in \overline{\mathfrak{a}^{+}}$such that $t_{j} \rightarrow+\infty,\left|H_{j}\right|=\mathrm{O}\left(t_{j}\right)$ and

$$
\inf _{j}\left|\frac{h_{t_{j}}\left(\exp H_{j}\right)}{t_{j}^{-\frac{\ell}{2}-\left|\Sigma^{++}\right|} \varphi_{0}\left(\exp H_{j}\right) e^{-|\varrho|^{2} t_{j}-\left(\left|H_{j}\right|^{2} / 4 t_{j}\right)}}-c_{5}\right|>0 .
$$

By passing to subsequences as above, we can assume that $\left|H_{j}\right| / \sqrt{t_{j}} \rightarrow+\infty$, because of (5.1.6), and that $H_{j} /\left|H_{j}\right|$ tends to a unit vector in some face $\left(\mathfrak{a}^{F}\right)^{+}$, by compactness and because of case (i). Under these assumptions, we can expand the heat kernel as in Steps 4 and 8 of section 3:

$$
h_{t_{j}}\left(\exp H_{j}\right)=\underbrace{I_{0}^{F}+I I_{0}^{F}+I I I_{0}^{F}}_{E_{0}^{F}}+\sum_{\substack{q \in 2 Q^{F} \\ 0<\varkappa(q)<N}} E_{q}^{F}+R_{N}^{F},
$$

with leading term

$$
\begin{aligned}
I_{0}^{F}=c_{2} \pi^{\frac{\ell^{F}}{2}}\left|W_{F}\right|^{-1} & t_{j}^{-\frac{\ell^{F}}{2}} \mathbf{c}^{F}\left(-i \frac{H_{j}^{F}}{2 t_{j}}\right)^{-1} e^{-|\varrho|^{2} t_{j}-\left\langle\varrho^{F}, H_{j}\right\rangle-\frac{\left|H_{j}^{F}\right|^{2}}{4 t_{j}}} \\
& \times \int_{\mathfrak{a}_{F}} d \lambda_{F}\left|\mathbf{c}_{F}\left(\lambda_{F}\right)\right|^{-2} e^{-t_{j}\left|\lambda_{F}\right|^{2}} \varphi_{\lambda_{F}}^{F}\left(\exp H_{j}\right)
\end{aligned}
$$


and remainder estimates

$$
\begin{gathered}
I I_{0}^{F}+I I I_{0}^{F}=\mathrm{o}\left(t_{j}^{-\frac{\ell}{2}-\left|\Sigma_{F}^{++}\right|}\left\{\prod_{\alpha \in \Sigma^{++}}\left(1+\left\langle\alpha, H_{j}\right\rangle\right)\right\} e^{-|\varrho|^{2} t_{j}-\left\langle\varrho, H_{j}\right\rangle-\frac{\left|H_{j}\right|^{2}}{4 t_{j}}}\right), \\
\sum_{\substack{q \in 2 Q^{F} \\
0<\varkappa(q)<N}} E_{q}^{F}+R_{N}^{F}=\mathrm{O}\left(t_{j}^{-\infty} e^{-|\varrho|^{2} t_{j}-\left\langle\varrho, H_{j}\right\rangle-\frac{\left|H_{j}\right|^{2}}{t_{j}}}\right) .
\end{gathered}
$$

According to the induction hypothesis, the integral in (5.1.8) behaves asymptotically like

$$
2^{-\left|\Sigma_{F}^{++}\right|} \pi^{\frac{\ell_{F}}{2}}\left|W_{F}\right| \boldsymbol{\pi}_{F}(\widetilde{\varrho}) \mathbf{b}_{F}(0)^{-2} t_{j}^{-\frac{\ell_{F}}{2}-\left|\Sigma_{F}^{++}\right|} \varphi_{0}^{F}\left(\exp H_{j}\right) e^{-\frac{\left|\left(H_{j}\right)_{F}\right|^{2}}{4 t_{j}}} .
$$

Putting everything together, we obtain

$$
\begin{aligned}
h_{t_{j}}\left(\exp H_{j}\right) \sim c_{2} 2^{-\left|\Sigma^{++}\right|} \pi^{\frac{\ell}{2}} \boldsymbol{\pi}_{F}(\widetilde{\varrho}) \mathbf{b}_{F}(0)^{-2} \mathbf{b}^{F}\left(-i \frac{H_{j}}{2 t_{j}}\right)^{-1} \\
\quad \times t_{j}^{-\frac{\ell}{2}-\left|\Sigma^{++}\right|} \boldsymbol{\pi}^{F}\left(H_{j}^{F}\right) \varphi_{0}^{F}\left(\exp H_{j}\right) e^{-|\varrho|^{2} t_{j}-\left\langle\varrho^{F}, H_{j}\right\rangle-\frac{\left|H_{j}\right|^{2}}{4 t_{j}}},
\end{aligned}
$$

which agrees with the asymptotics in Theorem 5.1.1, as observed at the end of (5.1.6). This contradicts (5.1.7) and concludes the proof of Theorem 5.1.1.

\subsection{Bessel-Green-Riesz kernels $\boldsymbol{k}_{\zeta}, \boldsymbol{\sigma}$.}

Theorem 5.2.1. (i) Let $\zeta>0$ and $\sigma>0$. Then the following asymptotics hold, for all sequences $H_{j} \in \overline{\mathfrak{a}^{+}}$tending to infinity, i.e. such that $\left|H_{j}\right| \rightarrow+\infty$ :

$k_{\zeta, \sigma}\left(\exp H_{j}\right) \sim c_{6} \mathbf{b}\left(-i \zeta \frac{H_{j}}{\left|H_{j}\right|}\right)^{-1}\left|H_{j}\right|^{\frac{\sigma-\ell-1}{2}-\left|\Sigma^{++}\right|} \varphi_{0}\left(\exp H_{j}\right) e^{-\zeta\left|H_{j}\right|}$ as $j \rightarrow+\infty$, where $c_{6}=c_{2} 2^{\frac{\ell+1-\sigma}{2}} \pi^{\frac{\ell+1}{2}} \boldsymbol{\pi}(\varrho) \mathbf{b}(0)^{-1} \Gamma\left(\frac{\sigma}{2}\right)^{-1} \zeta^{\frac{\ell-\sigma-1}{2}+\left|\Sigma^{++}\right|}$is a positive constant.

(ii) In the limit case $\zeta=0$ and for $0<\sigma<\ell+2\left|\Sigma^{++}\right|$, we have instead

$$
k_{0, \sigma}\left(\exp H_{j}\right) \sim c_{7}\left|H_{j}\right|^{\sigma-\ell-2\left|\Sigma^{++}\right|} \varphi_{0}\left(\exp H_{j}\right),
$$

with $c_{7}=c_{2} 2^{\ell+\left|\Sigma^{++}\right|-\sigma} \pi^{\frac{\ell}{2}} \boldsymbol{\pi}(\widetilde{\varrho}) \mathbf{b}(0)^{-2} \Gamma\left(\frac{\ell-\sigma}{2}+\left|\Sigma^{++}\right|\right) \Gamma\left(\frac{\sigma}{2}\right)^{-1}$.

REMARK 5.2.2. (i) The expressions $\mathbf{b}\left(-i \zeta \frac{H_{j}}{\left|H_{j}\right|}\right)^{-1}$ and $\varphi_{0}\left(\exp H_{j}\right)$ entering these asymptotics are positive and their behavior was recalled in subsection 2.2. For instance, let us specialize Theorem 5.2.1 to sequences $H_{j} \in \overline{\mathfrak{a}^{+}}$tending to infinity as in Proposition 2.2.12.ii:

$$
\left\{\begin{array}{l}
\left\langle\alpha, H_{j}\right\rangle=\mathrm{o}\left(\omega^{F}\left(H_{j}\right)\right) \quad \forall \alpha \in F, \\
\omega^{F}\left(H_{j}\right)=\min _{\alpha \in \Sigma^{+++} \backslash F}\left\langle\alpha, H_{j}\right\rangle \rightarrow+\infty,
\end{array}\right.
$$

where $F$ is a proper subset of $\Sigma^{+++}$(possibly empty). Then 
- $\zeta>0$ and $\sigma>0$ :

$$
k_{\zeta, \sigma}\left(\exp H_{j}\right) \sim
$$

$c_{6}^{F} \mathbf{b}^{F}\left(-i \zeta \frac{H_{j}}{\left|H_{j}\right|}\right)^{-1}\left|H_{j}\right|^{\frac{\sigma-\ell-1}{2}-\left|\Sigma^{++}\right|} \boldsymbol{\pi}^{F}\left(H_{j}\right) \varphi_{0}^{F}\left(\exp H_{j}\right) e^{-\left\langle\varrho^{F}, H_{j}\right\rangle-\zeta\left|H_{j}\right|}$,

with $c_{6}^{F}=c_{2} 2^{\frac{\ell+1-\sigma}{2}} \pi^{\frac{\ell+1}{2}} \boldsymbol{\pi}_{F}(\widetilde{\varrho}) \mathbf{b}_{F}(0)^{-2} \Gamma\left(\frac{\sigma}{2}\right)^{-1} \zeta^{\frac{\ell-\sigma-1}{2}+\left|\Sigma^{++}\right|}$,

- $\zeta=0$ and $0<\sigma<\ell+2\left|\Sigma^{++}\right|$:

$$
k_{0, \sigma}\left(\exp H_{j}\right) \sim c_{7}^{F}\left|H_{j}\right|^{\sigma-\ell-2\left|\Sigma^{++}\right|} \varphi_{0}\left(\exp H_{j}\right)
$$

with $c_{7}^{F}=c_{2} 2^{\ell+\left|\Sigma^{++}\right|-\sigma} \pi^{\frac{\ell}{2}} \boldsymbol{\pi}_{F}(\widetilde{\varrho}) \mathbf{b}(0)^{-1} \mathbf{b}_{F}(0)^{-1} \Gamma\left(\frac{\ell-\sigma}{2}+\left|\Sigma^{++}\right|\right) \Gamma\left(\frac{\sigma}{2}\right)^{-1}$.

(ii) When $G$ is complex, the behavior of $k_{\zeta, \sigma}$ can be easily read off from the explicit formulas

- $\zeta>0$ and $\sigma>0$ :

$$
k_{\zeta, \sigma}(x)=2^{1-\frac{n+\sigma}{2}} \pi^{-\frac{n}{2}} \Gamma\left(\frac{\sigma}{2}\right)^{-1} \zeta^{\frac{n-\sigma}{2}}|x|^{\frac{\sigma-n}{2}} \varphi_{0}(x) K_{ \pm \frac{n-\sigma}{2}}(\zeta|x|),
$$

- $\zeta=0$ and $0<\sigma<n$ :

$$
k_{0, \sigma}=2^{-\sigma} \pi^{-\frac{n}{2}} \Gamma\left(\frac{n-\sigma}{2}\right) \Gamma\left(\frac{\sigma}{2}\right)^{-1}|x|^{\sigma-n} \varphi_{0}(x),
$$

for $x \in G \backslash K$, which are obtained by combining (4.2.4.2) with Remark 5.1.2.iii. Here

$$
\varphi_{0}(\exp H)=\prod_{\alpha \in \Sigma^{+}} \frac{\langle\alpha, H\rangle}{\sinh \langle\alpha, H\rangle} \quad \forall H \in \mathfrak{a}
$$

and $K_{\nu}(z)$ denotes the classical Bessel $K$-function, also known as modified Bessel function of the third kind or Macdonald's function (see for instance [L, Ch.45]).

(iii) In rank 1, the asymptotics in Theorem 5.2.1 read as follows, resuming the notation of Remark 5.1.2.iv:

- $\zeta>0$ and $\sigma>0$ :

$$
k_{\zeta, \sigma}\left(\exp r_{j} H_{0}\right) \sim c_{6}^{\prime} r_{j}^{\frac{\sigma}{2}-1} e^{-(\varrho+\kappa \zeta) r_{j}},
$$

with $c_{6}^{\prime}=2^{m_{2 \alpha}+1-\frac{\sigma}{2}} \pi^{\frac{1-n}{2}} \kappa^{\frac{\sigma}{2}+1-n} \Gamma\left(\frac{\sigma}{2}\right)^{-1} \zeta^{1-\frac{\sigma}{2}} \gamma(\zeta)$,

- $\zeta=0$ and $0<\sigma<3$ :

$$
k_{0, \sigma}\left(\exp r_{j} H_{0}\right) \sim c_{7}^{\prime} r_{j}^{\sigma-2} e^{-\varrho r_{j}},
$$

with $c_{7}^{\prime}=2^{\varrho+1-\sigma} \pi^{-\frac{n+1}{2}} \kappa^{\sigma-n} \Gamma\left(\frac{\varrho}{2}\right) \Gamma\left(\frac{m_{\alpha}}{4}+\frac{1}{2}\right) \Gamma\left(\frac{3-\sigma}{2}\right) \Gamma\left(\frac{\sigma}{2}\right)^{-1}$.

These results extend again to Damek-Ricci spaces.

(iv) Let us specialize Theorem 5.2.1 to the Green function $k_{\zeta}=k_{\zeta, 2}$ :

- $\zeta>0$ :

$$
k_{\zeta}\left(\exp H_{j}\right) \sim c_{6}^{\prime \prime} \mathbf{b}\left(-i \zeta \frac{H_{j}}{\left|H_{j}\right|}\right)^{-1}\left|H_{j}\right|^{\frac{1-\ell}{2}-\left|\Sigma^{++}\right|} \varphi_{0}\left(\exp H_{j}\right) e^{-\zeta\left|H_{j}\right|},
$$

with $c_{6}^{\prime \prime}=c_{2} 2^{\frac{\ell-1}{2}} \pi^{\frac{\ell+1}{2}} \boldsymbol{\pi}(\widetilde{\varrho}) \mathbf{b}(0)^{-1} \zeta^{\frac{\ell-3}{2}+\left|\Sigma^{++}\right|}$, 
- $\zeta=0$ :

$$
k_{0}\left(\exp H_{j}\right) \sim c_{7}^{\prime \prime}\left|H_{j}\right|^{2-\ell-2\left|\Sigma^{++}\right|} \varphi_{0}\left(\exp H_{j}\right),
$$

with $c_{7}^{\prime \prime}=c_{2} 2^{\ell+\left|\Sigma^{++}\right|-2} \pi^{\frac{\ell}{2}} \Gamma\left(\frac{\ell}{2}+\left|\Sigma^{++}\right|-1\right) \boldsymbol{\pi}(\widetilde{\varrho}) \mathbf{b}(0)^{-2}$.

Recall that these asymptotics are closely related to the Martin compactification of $\mathbb{X}=G / K$ (see [GuJT2] and the references cited therein). Complete Green function asymptotics had been obtained previously in some particular cases: when $G$ is complex ([Dy], [No]), when $\mathbb{X}$ is a product of hyperbolic spaces [GiW], or when $G$ is real normal, i.e. totally split [Cha]. (Notice by comparison that $\mathbf{b}(-i \lambda)^{-1}$ coincides, up to a positive constant, with the expression $\kappa(\lambda)=\int_{K_{\mathbb{C}}} d h e^{-\langle\varrho+\lambda, H(h)\rangle}$ used in [Cha].) The general case was actually announced a long time ago in [Ol1] (see also Remark 2.2.13.iv). But the delicate analysis along faces relied on a misuse of Harish-Chandra's expansion (recalled in Theorem 2.2.7), as came out in the preprint version of [Ol3]. In the final version, asymptotics of $k_{\zeta}\left(\exp H_{j}\right)$ are stated in [Ol3, Theorem 3.2] under the following restricted assumptions: $G$ is semisimple, $\zeta>0$ and

$$
\left\{\begin{array}{l}
H_{j} \in \overline{\mathfrak{a}^{+}} \text {tends to infinity i.e. }\left|H_{j}\right| \rightarrow+\infty, \\
\left(H_{j}\right)_{F} \text { tends to a nonzero vector in } \overline{\left(\mathfrak{a}_{F}\right)^{+}}, \\
\frac{H_{j}}{\left|H_{j}\right|} \text { i.e. } \frac{\left(H_{j}\right)^{F}}{\left|\left(H_{j}\right)^{F}\right|} \text { tends to a unit vector in } \overline{\left(\mathfrak{a}^{F}\right)^{+}},
\end{array}\right.
$$

for some proper subset $F$ of $\Sigma^{+++}$. But the proof requires actually more, namely

$$
H_{j} \in \mathfrak{a}^{+}, \quad \lim \left(H_{j}\right)_{F} \in\left(\mathfrak{a}_{F}\right)^{+} \quad \text { and } \quad \lim \frac{\left(H_{j}\right)^{F}}{\left|\left(H_{j}\right)^{F}\right|} \in\left(\mathfrak{a}^{F}\right)^{+} .
$$

The first two conditions come from the Harish-Chandra expansion. The last condition is needed to ensure the nonvanishing of the expressions $c_{z}\left(-i a^{\prime} \frac{\widetilde{\mathfrak{r}}}{|\mathfrak{r}|}\right)^{-1}$ in $[\mathrm{Ol} 3,(3.47)]$ and $c_{z}\left(-i a \frac{\widetilde{\mathfrak{r}}}{|\mathfrak{r}|}\right)^{-1}$ in $[\mathrm{Ol} 3,(3.51)]$. Otherwise the asymptotic analysis carried out in [Ol3, Section 3] yields no leading term and is not conclusive. Thus the gap in Olshanetsky's asymptotics of $k_{\zeta}\left(\exp H_{j}\right)$ consists essentially in the cases where

$$
\left\{\begin{array}{l}
\text { some }\left\langle\alpha, H_{j}\right\rangle \text { tend to } 0 \\
\text { some }\left\langle\alpha, H_{j}\right\rangle \text { tend to }+\infty \text { at different rates }
\end{array}\right.
$$

Finally, let us mention that Green potential asymptotics have also been obtained for random walks on $\mathbb{X}$, along directions $x_{j}=k_{j}\left(\exp H_{j}\right) K$ with radial part

$$
\left\{\begin{array}{l}
H_{j} \in \mathfrak{a}^{+} \\
\left|H_{j}\right| \rightarrow+\infty \\
\left\langle\alpha, H_{j}\right\rangle \asymp\left|H_{j}\right| \quad \forall \alpha \in \Sigma^{+(++)}
\end{array}\right.
$$


$([\mathrm{B} 1,3],[\mathrm{Bo} 1,4])$.

The proof of Theorem 5.2.1 relies on the material developed in subsections 4.2 and 5.1. Recall the formula

$$
k_{\zeta, \sigma}\left(\exp H_{j}\right)=\frac{1}{\Gamma\left(\frac{\sigma}{2}\right)} \int_{0}^{+\infty} \frac{d t}{t} t^{\frac{\sigma}{2}} e^{\left(|\varrho|^{2}-\zeta^{2}\right) t} h_{t}\left(\exp H_{j}\right) .
$$

(i) When $\zeta>0$, we know that the main contribution in (4.2.4.2) comes from the integral $\int_{\kappa^{-1} \frac{\left|H_{j}\right|}{2 \zeta}}^{\kappa \frac{\left|H_{j}\right|}{2 \zeta}}$ around $\frac{\left|H_{j}\right|}{2 \zeta}$, which yields

$$
\begin{gathered}
\Gamma\left(\frac{\sigma}{2}\right)^{-1} \varphi_{0}\left(\exp H_{j}\right) \int_{\kappa^{-1} \frac{\left|H_{j}\right|}{2 \zeta}}^{\kappa \frac{\left|H_{j}\right|}{2 \zeta}} d t t^{\frac{\sigma-\ell}{2}-\left|\Sigma^{++}\right|-1} \mathbf{b}\left(-i \frac{H_{j}}{2 t}\right)^{-1} e^{-\zeta^{2} t-\frac{\left|H_{j}\right|^{2}}{4 t}} h\left(t, H_{j}\right) \\
=\Gamma\left(\frac{\sigma}{2}\right)^{-1}\left(\frac{\left|H_{j}\right|}{2 \zeta}\right)^{\frac{\sigma-\ell}{2}-\left|\Sigma^{++}\right|} \mathbf{b}\left(-i \zeta \frac{H_{j}}{\left|H_{j}\right|}\right)^{-1} \varphi_{0}\left(\exp H_{j}\right) \\
\times \int_{\kappa^{-1}}^{\kappa} d t e^{-\zeta\left|H_{j}\right| \frac{t+t^{-1}}{2}} t^{\frac{\sigma-\ell}{2}-\left|\Sigma^{++}\right|-1} \frac{\mathbf{b}\left(-i \zeta \frac{H_{j}}{\left|H_{j}\right|}\right)}{\mathbf{b}\left(-i \zeta \frac{H_{j}}{\left|H_{j}\right|} t^{-1}\right)} h\left(\frac{\left|H_{j}\right|}{2 \zeta} t, H_{j}\right),
\end{gathered}
$$

where

$$
h(t, H)=t^{\frac{\ell}{2}+\mid \Sigma^{++}} \mathbf{b}\left(-i \frac{H}{2 t}\right) \varphi_{0}(\exp H)^{-1} e^{|\varrho|^{2} t+\frac{|H|^{2}}{4 t}} h_{t}(\exp H) .
$$

The asymptotic behavior

$$
I_{j} \sim c_{5} \sqrt{\frac{2 \pi}{\zeta}}\left|H_{j}\right|^{-\frac{1}{2}} e^{-\zeta\left|H_{j}\right|}
$$

of the last integral is obtained by the Laplace method. Notice that

(5.2.3.1) $\mathbf{b}\left(-i \zeta \frac{H_{j}}{\left|H_{j}\right|} t^{-1}\right)$ is bounded above and below, uniformly in $j$ and $t$,

(5.2.3.2) $\mathbf{b}\left(-i \zeta \frac{H_{j}}{\left|H_{j}\right|} t^{-1}\right) \sim \mathbf{b}\left(-i \zeta \frac{H_{j}}{\left|H_{j}\right|}\right)$ as $t \rightarrow 1$, uniformly in $j$,

(5.2.3.3) $h\left(\frac{\left|H_{j}\right|}{2 \zeta} t, H_{j}\right) \rightarrow c_{5}$ as $j \rightarrow+\infty$, uniformly in $t$.

While (5.2.3.1) and (5.2.3.2) are elementary, (5.2.3.3) follows from Theorem 5.1 .1 by contradiction.

(ii) When $\zeta=0$, the main contribution in (4.2.4.2) comes instead from the integral $\int_{\left|H_{j}\right|^{2-\eta}}^{\left|H_{j}\right|^{2+\eta}}$ around $\left|H_{j}\right|^{2}$, which yields

$$
\begin{aligned}
\Gamma\left(\frac{\sigma}{2}\right)^{-1} \varphi_{0}\left(\exp H_{j}\right) \int_{\left|H_{j}\right|^{2-\eta}}^{\left|H_{j}\right|^{2+\eta}} \frac{d t}{t} t^{\frac{\sigma-\ell}{2}-\left|\Sigma^{++}\right|} e^{-\frac{\left|H_{j}\right|^{2}}{4 t}} h^{\prime}\left(t, H_{j}\right) \\
=\Gamma\left(\frac{\sigma}{2}\right)^{-1}\left(\frac{\left|H_{j}\right|}{2}\right)^{\sigma-\ell-2\left|\Sigma^{++}\right|} \varphi_{0}\left(\exp H_{j}\right) \\
\times \int_{\frac{1}{4}\left|H_{j}\right|^{-\eta}}^{\frac{1}{4}\left|H_{j}\right|^{\eta}} \frac{d t}{t} t^{\frac{\ell-\sigma}{2}+\left|\Sigma^{++}\right|} e^{-t} h^{\prime}\left(\frac{\left|H_{j}\right|^{2}}{4 t}, H_{j}\right) .
\end{aligned}
$$


Here

$$
h^{\prime}\left(t, H_{j}\right)=t^{\frac{\ell}{2}+\left|\Sigma^{++}\right|} \varphi_{0}\left(\exp H_{j}\right)^{-1} e^{|\varrho|^{2} t+\frac{\left|H_{j}\right|^{2}}{4 t}} h_{t}\left(\exp H_{j}\right)
$$

tends uniformly to $c_{5} \mathbf{b}(0)^{-1}$, as $\left|H_{j}\right|^{2-\eta} \leq t \leq\left|H_{j}\right|^{2+\eta}$ and $j \rightarrow+\infty$. This follows from Theorem 5.1.1.i, again by contradiction. As a conclusion, the last integral tends to $c_{5} \mathbf{b}(0)^{-1} \Gamma\left(\frac{\ell-\sigma}{2}+\left|\Sigma^{++}\right|\right)$.

\subsection{Poisson kernel $p_{t}, \zeta$.}

Theorem 5.3.1. (i) Assume $\zeta>0$. Let $t_{j} \in(0,+\infty)$ and $H_{j} \in \overline{\mathfrak{a}^{+}}$be two sequences such that $t_{j}+\left|H_{j}\right| \rightarrow+\infty$. Then

$$
\begin{aligned}
p_{t, \zeta}\left(\exp H_{j}\right) \sim c_{8} \mathbf{b} & \left(-i \zeta \frac{H_{j}}{\sqrt{t_{j}^{2}+\left|H_{j}\right|^{2}}}\right)^{-1} \\
& \times t_{j}\left(t_{j}^{2}+\left|H_{j}\right|^{2}\right)^{-\frac{\ell}{4}-\frac{1}{2}\left|\Sigma^{++}\right|-\frac{1}{2}} \varphi_{0}\left(\exp H_{j}\right) e^{-\zeta \sqrt{t_{j}^{2}+\left|H_{j}\right|^{2}}},
\end{aligned}
$$

where $c_{8}=c_{2} 2^{\frac{\ell}{2}} \pi^{\frac{\ell}{2}} \boldsymbol{\pi}(\widetilde{\varrho}) \mathbf{b}(0)^{-1} \zeta^{\frac{\ell}{2}+\left|\Sigma^{++}\right|}$is a positive constant.

(ii) In the limit case $\zeta=0$, we have instead

$$
p_{t, 0}\left(\exp H_{j}\right) \sim c_{9} t_{j}\left(t_{j}^{2}+\left|H_{j}\right|^{2}\right)^{-\frac{\ell}{2}-\left|\Sigma^{++}\right|-\frac{1}{2}} \varphi_{0}\left(\exp H_{j}\right),
$$

with $c_{9}=c_{2} 2^{\ell+\mid \Sigma^{++}} \pi^{\frac{\ell-1}{2}} \Gamma\left(\frac{\ell+1}{2}+\left|\Sigma^{++}\right|\right) \boldsymbol{\pi}(\widetilde{\varrho}) \mathbf{b}(0)^{-2}$.

Theorem 5.3.1 is proved like Theorem 5.2.1, using the material in Subsections 4.3 and 5.1 .

REMARK 5.3.2. We have again

(i) the following explicit expressions when $G$ is complex:

- $\zeta>0$ :

$$
\begin{gathered}
p_{t, \zeta}(x)=2^{-\frac{n-1}{2}} \pi^{-\frac{n+1}{2}} \zeta^{\frac{n+1}{2}} t\left(t^{2}+|x|^{2}\right)^{-\frac{n+1}{4}} \varphi_{0}(x) K_{ \pm \frac{n+1}{2}}\left(\zeta \sqrt{t^{2}+|x|^{2}}\right), \\
\bullet \zeta=0: \\
p_{t, 0}(x)=\pi^{-\frac{n+1}{2}} \Gamma\left(\frac{n+1}{2}\right) t\left(t^{2}+|x|^{2}\right)^{-\frac{n+1}{2}} \varphi_{0}(x),
\end{gathered}
$$

(ii) the following asymptotics in rank 1 :

- $\zeta>0$ :

$p_{t, \zeta}\left(\exp r_{j} H_{0}\right) \sim c_{8}^{\prime} \gamma\left(\zeta \frac{\kappa r_{j}}{\sqrt{t_{j}^{2}+\kappa^{2} r_{j}^{2}}}\right) t_{j} r_{j}\left(t_{j}^{2}+\kappa^{2} r_{j}^{2}\right)^{-\frac{5}{4}} e^{-\varrho r_{j}-\zeta \sqrt{t_{j}^{2}+\kappa^{2} r_{j}^{2}}}$

where $c_{8}^{\prime}=2^{m_{2 \alpha}+\frac{1}{2}} \pi^{-\frac{n}{2}} \kappa^{3-n} \zeta^{\frac{3}{2}}$,

- $\zeta=0$ :

$$
p_{t, 0}\left(\exp r_{j} H_{0}\right) \sim c_{9}^{\prime} t_{j} r_{j}\left(t_{j}^{2}+\kappa^{2} r_{j}^{2}\right)^{-2} e^{-\varrho r_{j}},
$$

where $c_{9}^{\prime}=2^{\varrho+1} \pi^{-\frac{n}{2}-1} \kappa^{3-n} \Gamma\left(\frac{\varrho}{2}\right) \Gamma\left(\frac{m_{\alpha}}{4}+\frac{1}{2}\right)$, which extend straightforwardly to Damek-Ricci spaces. 


\section{References}

[A] P. Albuquerque, Mesures de Patterson-Sullivan dans les espaces symétriques de rang supérieur, Thèse, Universités de Genève et de Lausanne (1997).

[An1] J.-Рн. ANker, La forme exacte de l'estimation fondamentale de Harish-Chandra, C.R. Acad. Sci. Paris Série I, 305 (1987), 371-374.

[An2] J.-PH. ANker, Le noyau de la chaleur sur les espaces symétriques $\mathrm{U}(p, q) / \mathrm{U}(p) \times \mathrm{U}(q)$ in "Harmonic Analysis, Luxembourg 1987" (P. Eymard, J.-P. Pier, eds.), Springer Lect. Notes Math. 1359 (1988), 60-82.

[An3] J.-PH. ANKER, $L^{p}$ Fourier multipliers on Riemannian symmetric spaces of the noncompact type, Ann. Math. 132 (1990), 597-628.

[An4] J.-PH. AnkeR, The spherical Fourier transform of rapidly decreasing functions / A simple proof of a characterization due to HarishChandra, Helgason, Trombi and Varadarajan, J. Funct. Anal. 96 (1991), 331-349.

[An5] J.-PH. ANkER, Sharp estimates for some functions of the Laplacian on noncompact symmetric spaces, Duke Math. J. 65 (1992), 257-297.

[AnDY] J.-Ph. Anker, E. Damek, C. Yacoub, Spherical analysis on harmonic $A N$ groups, Ann. Scuola Norm. Sup. Pisa XXIII (1996), 643679 .

[AnJ1] J.-Ph. Anker, L. Ji, Comportement exact du noyau de la chaleur et de la fonction de Green sur les espaces symétriques non compacts, C.R. Acad. Sci. Paris Série I 326 (1998), 153-156.

[AnJ2] J.-Ph. Anker, L. Ji, Heat kernel and Green function estimates on noncompact symmetric spaces II, in "Boundaries and Lie groups," (J.C. Taylor, ed.), CRM Proc. Conf. Series, Amer. Math. Soc., to appear.

[AnSe] J.-Ph. Anker, A.G. Setti, Asymptotic finite propagation speed for heat diffusion on certain Riemannian manifolds, J. Funct. Anal. 103 (1992), 50-61.

[B1] M. BABIllot, Potential at infinity on symmetric spaces and Martin boundary, in "Harmonic Analysis and Discrete Potential Theory, Frascati 1991," (M. Picardello, ed.), Plenum Press (1992), 23-46.

[B2] M. BABILlot, A probabilistic approach to heat diffusion on symmetric spaces, J. Theoretical Prob. 7 (1994), 599-607.

[B3] M. BABILlot, Asymptotics of Green functions on a class of solvable Lie groups, Potential Anal. 8 (1998), 69-100.

[Bo1] Ph. Bougerol, Comportement asymptotique des puissances de convolution d'une probabilité sur un espace symétrique, in "Journées sur les marches aléatoires, Nancy 1979" Astérisque 74, Soc. Math. France (1980), 29-45. 
[Bo2] Ph. Bougerol, Théorème central limite local sur certains groupes de Lie, Ann. Scient. Éc. Norm. Sup. 4e Sér., 14 (1981), 403-432.

[Bo3] Ph. Bougerol, Exemples de théorèmes locaux sur les groupes résolubles, Ann. Inst. Henri Poincaré Section B (Prob. Stat.) XIX (1983), 369-391.

[Bo4] Ph. Bougerol, Comportement à l'infini du noyau potentiel sur un espace symétrique, in "Théorie du potentiel, Orsay 1983" (G. Mokobodzki, D. Pinchon, eds.), Springer Lect. Notes Math. 1096 (1984), $90-115$.

[BrOSc] T. Branson, G. Ólafsson, H. Schlichtkrull, Huyghens' principle in Riemannian symmetric spaces, Math. Ann. 301 (1995), 445-462.

[CMi] W. Casselman, D. Miličič, Asymptotic behavior of matrix coefficients of admissible representations, Duke Math. J. 49 (1982), 869-930.

[Ch] I. Chavel, Eigenvalues in Riemannian Geometry, Academic Press, 1984.

[Cha] M. ChAYet, Le comportement asymptotique du noyau de Green dans le cas des groupes semi-simples déployés, preprint (1994).

[Cl] J.-L. ClerC, Bochner-Riesz means of $H^{p}$ functions $(0<p<1)$ on compact Lie groups, in "Non-commutative Harmonic Analysis and Lie Groups, Luminy 1985" (J. Carmona, et al., eds.), Springer Lect. Notes Math. 1243 (1987), 86-107.

[Co] E.T. Copson, Asymptotic Expansions, Cambridge Univ. Press, 1965.

[CowGGM] M.G. Cowling, G.I. Gaudry, S. Giulini, G. Mauceri, Weak type $(1,1)$ estimates for heat kernel maximal functions on Lie groups, Trans. Amer. Math. Soc. 323 (1991), 637-649.

[CowGHM] M.G. Cowling, S. Giulini, A. Hulanicki, G. Mauceri, Spectral multipliers for a distinguished Laplacian on certain groups of exponential growth, Studia Math. 111 (1994), 103-121.

[CowGM1] M.G. Cowling, S. Givlini, S. MedA, $L^{p}-L^{q}$ estimates for functions of the Laplace-Beltrami operator on noncompact symmetric spaces I, Duke Math. J. 72 (1993), 109-150.

[CowGM2] M.G. Cowling, S. Giulini, S. MedA, $L^{p}-L^{q}$ estimates for functions of the Laplace-Beltrami operator on noncompact symmetric spaces II, J. Lie Theory 5 (1995), 1-14.

[Da] E.B. Davies, Heat Kernels and Spectral Theory, Cambridge Univ. Press, 1989.

[DaM $] \quad$ E.B. Davies, N. Mandouvalos, Heat kernels bounds on hyperbolic space and Kleinian groups, Proc. London Math. Soc. 57 (1988), 182208.

[DeGav] A. Debiard, B. Gaveau, Analysis on root systems, Can. J. Math. XXXIX (1987), 1281-1404.

[DuKoV] J.J. Duistermaat, J.A.C. Kolk, V.S. Varadarajan, Spectra of 
compact locally symmetric manifolds of negative curvature, Invent. Math. 52 (1979), 27-93.

[DunSch] N. Dunford, J.T. Schwartz, Linear Operators I, Interscience, 1958.

[Dy] E.B. Dynkin, Brownian motion in certain symmetric spaces and non-negative eigenfunctions of the Laplace-Beltrami operator, Amer. Math. Soc. Transl. Ser. 2, 72 (1968), 203-228.

[G] R. Gangolli, Asymptotic behaviour of spectra of compact quotients of certain symmetric spaces, Acta Math. 121 (1968), 151-192.

[GV] R. Gangolli, V.S. Varadarajan, Harmonic Analysis of Spherical Functions on Real Reductive Groups, Springer-Verlag, 1988.

[GaSj] G. Gaudry, P. SJögren, Singular integrals on Iwasawa $N A$ groups of rank 1, J. reine angew. Math. 479 (1996), 39-66.

[GiMau] S. Giulini, G. Mauceri, Analysis of a distinguished Laplacian on solvable Lie groups, Math. Nachr. 163 (1993), 151-162.

[GiW] S. Giulini, W. Woess, The Martin compactification of the Cartesian product of two hyperbolic spaces, J. reine angew. Math. 444 (1993), $17-28$.

[Gr] A. Grigor'yan, Heat kernel of a noncompact Riemannian manifold, in "Stochastic Analysis, (Summer Research Institute, Cornell University, July 1993)" (M.C. Cranston, M.A. Pinsky, eds.), Proc. Symp. Pure Math. 57, Amer. Math. Soc. (1995), 239-263.

[Gu] Y. Guivarc'H, Compactifications of symmetric spaces and positive eigenfunctions of the Laplacian, Sém. Math. Univ. Rennes (1995), $67 \mathrm{pp}$.

[GuJT1] Y. GuivarC'H, L. Ji, J.C. TAYLOR, Compactifications of symmetric spaces, C.R. Acad. Sci. Paris Sér. I, 317 (1993), 1103-1108.

[GuJT2] Y. Guivarc'H, L. Ji, J.C. TAYlor, Compactifications of Symmetric Spaces, Progress Math. 156, Birkhäuser, 1998.

[H] W. HeBisch, The subalgebra of $L^{1}(A N)$ generated by the Laplacian, Proc. Amer. Math. Soc. 117 (1993), 547-549.

[He1] S. Helgason, Differential Geometry, Lie Groups, and Symmetric Spaces, Academic Press 1978.

[He2] S. Helgason, Groups and Geometric Analysis, Academic Press, 1984.

[He3] S. Helgason, Geometric Analysis on Symmetric Spaces, Amer. Math. Soc. 1994.

[Hu $\quad$ A. Hulanicki, On the spectrum of the Laplacian on the affine group of the real line, Studia Math. LIV (1976), 199-204.

[K] Y. KanNAI, Off diagonal short time asymptotics for fundamental solutions of diffusion equations, Comm. P.D.E. 2 (1977), 781-830.

[L] N.N. Lebedev, Special Functions and their Applications, Dover, 1972.

[LiY] P. LI, S.T. YAU, On the parabolic kernel of the Schrödinger operator, 
Acta Math. 156 (1986), 153-201.

[Lo1] N. Lohoué, Comparaison de plusieurs fonctions de Littlewood-Paley, C.R. Acad. Sci. Paris Sér. I, 302 (1986), 339-342.

[Lo2] N. Lohoué, Estimations des fonctions de Littlewood-Paley-Stein sur les variétés Riemanniennes à courbure non positive, Ann. Scient. Éc. Norm. Sup. $4^{\text {e }}$ Sér., 20 (1987), 505-544.

[Lo3] N. Lohoué, Transformées de Riesz et fonctions de Littlewood-Paley sur les groupes non moyennables, C.R. Acad. Sci. Paris Série I, 306 (1988), 327-330.

[Lo4] N. Lohoué, Transformées de Riesz et fonctions sommables, Amer. J. Math. 114 (1992), 875-922.

[Lo5] N. LohouÉ, Estimations de certaines fonctions maximales et des transformées de Riesz multiples sur les variétés de Cartan-Hadamard et les groupes unimodulaires, C.R. Acad. Sci. Paris Série I, 312 (1991), $561-566$.

[LorRo] G. Lorang, B. Roynette, Etude d'une fonctionnelle liée au pont de Bessel, Ann. Inst. Henri Poincaré Section B (Prob. Stat.) 32 (1996), 107-133.

[Lu] Q. Lu, The heat kernel of non-compact symmetric spaces, Lect. Notes, Autumn School, "Analysis and Geometry on Complex Homogeneous Domains and Related Topics," Beijing 1997.

[MaNSt] G.A. Margulis, A. Nevo, E.M. Stein, Analogs of Wiener's ergodic theorems for semi-simple Lie groups II, preprint (1998).

[Mn] R. Mneimné, Equation de la chaleur sur un espace Riemannien symétrique et formule de Plancherel, Bull. Sc. Math. 2e Sér. 107 (1983), 261-287.

[No] E. NoldE, Nonnegative eigenfunctions of Beltrami-Laplace operator on symmetric spaces with complex semi-simple Lie group, Usp. Math. Nauk 24 (1969), 189-190.

[Ol1] M.A. Olshanetsky, The Martin boundaries of symmetric spaces with nonpositive curvature, Usp. Math. Nauk. 24 (1969), 189-190.

[Ol2] M.A. Olshanetsky, Asymptotics of spherical functions, Usp. Math. Nauk. 27 (1972), 211-212.

[O13] M.A. Olshanetsky, Martin boundaries for real semisimple Lie groups, J. Funct. Anal. 126 (1994), 169-216.

[R] D.W. Robinson, Elliptic Operators and Lie Groups, Clarendon Press \& Oxford Univ. Press, 1991.

[Sa1] P. SAWYER, The heat equation on the spaces of positive definite matrices, Can. J. Math. 44 (1992), 624-651.

[Sa2] P. SAWYER, The inverse of the Abel transform on $\mathrm{SU}^{*}(2 n) / \mathrm{Sp}(n)$, preprint (1992).

[Sa3] P. SAWYER, On an upper bound for the heat kernel on $\mathrm{SU}^{*}(2 n) / \operatorname{Sp}(n)$, 
Can. Math. Bull. 37 (1994), 408-418.

[Sa4] P. SAWYER, Estimates for the heat kernel on $\mathrm{SL}(n, \mathbb{R}) / \mathrm{SO}(n)$, Can. J. Math. 49 (1997), 359-372.

[St] E.M. Stein, Topics in Harmonic Analysis Related to the LittlewoodPaley Theory, Ann. Math. Stud. 63, Princeton Univ. Press, 1970.

[Str] J.-O. StrömberG, Weak type $L^{1}$ estimates for maximal functions on non-compact symmetric spaces, Ann. Math. 114 (1981), 115-126.

[TrV] P.C. Trombi, V.S. Varadarajan, Spherical transforms on semisimple Lie groups, Ann. Math. 94 (1971), 246-303.

[Va] N.Th. Varopoulos, Analysis on Lie groups, Rev. Mat. Iberoam. 12 (1996), 791-917.

[VaSCou] N.Th. Varopoulos, L. Saloff-Coste, T. Coulhon, Analysis and Geometry on Groups, Cambridge Univ. Press, 1992.

Jean-Philippe Anker

Inst. de Math. Elie Cartan

(Laboratoire Commun UHP-CNRS-INRIA)

Université Henri Poincaré (Nancy I)

B.P. 239

F-54506 Vandoeuvre-lès-Nancy Cedex

France

anker@iecn.u-nancy.fr
Lizhen Ji

Dept. of Math.

University of Michigan

East Hall

525 East University Avenue

Ann Arbor, MI 48109-1109

USA

lji@math. Isa.umich.edu

Submitted: August 1998

Final version: October 1999 